\title{
HEBEPHILIC SEXUAL INTERESTS IN SEXUAL OFFENDERS
}

\author{
by \\ Skye Mary-Jean Stephens \\ Master of Arts in Psychology, Ryerson University, 2012 \\ Bachelor of Arts in Psychology and Criminology, York University, April 2009
}

A Dissertation presented to Ryerson University

In partial fulfillment of the requirements for the degree of Doctor of Philosophy in the Program of Psychology

Toronto, Ontario, Canada, 2016

(OSkye Stephens 2016 


\section{Author's Declaration Page}

I hereby declare that I am the sole author of this dissertation. This is a true copy of the dissertation, including any required final revisions, as accepted by my examiners.

I authorize Ryerson University to lend this dissertation to other institutions or individuals for the purpose of scholarly research

I further authorize Ryerson University to reproduce this dissertation by photocopying or by other means, in total or in part, at the request of other institutions or individuals for the purpose of scholarly research.

I understand that my dissertation may be made electronically available to the public. 


\begin{abstract}
Hebephilic Sexual Interests in Sexual Offenders
\end{abstract}

Skye Stephens

Doctor of Philosophy in the Program of Psychology, 2012

Ryerson University

Hebephilia refers to a sexual interest for pubescent children in Tanner Stage 2 or 3 of sexual development (i.e., early stages of secondary sex development; Blanchard, Lykins, et al., 2009). Hebephilia was recently proposed as a standalone diagnosis, alongside pedophilia, in the main diagnostic manual used by clinicians in Canada and the United States to diagnose mental health disorders, which ignited considerable controversy in the field. The dissertation examined the validity of hebephilia as a construct through three interrelated studies utilizing a large sample

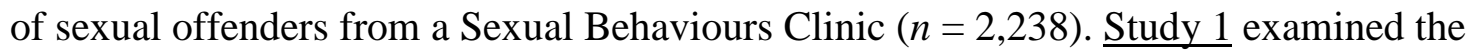
convergent validity of hebephilia in a sample of admitters and deniers. There was convergence among self-report, sexual behaviour, and sexual arousal indicators of hebephilia. Further, there was considerable overlap between pedophilia (i.e., sexual interest in prepubescent children) and hebephilia. Study 2 and $\underline{\text { Study } 3}$ examined the association between hebephilia and sexual offending. Hebephilia is arguably most pertinent to furthering our understanding of sexual offending against children, as one could not act on this sexual interest legally without committing a sexual offence against a child. Study 2 examined concurrent validity by examining the role of hebephilia in sexual victim choice. Similar to pedophilia, hebephilia was associated with a greater number of victims under the age of 15 . Further, hebephilia was associated with an increased likelihood of victim age polymorphism (i.e., having victims in other age ranges). Finally, Study 3 examined a subsample of offenders assessed at the Sexual Behaviours Clinic (n 
$=656$ ) whose criminal records were coded and analyzed in order to assess the predictive validity of hebephilia. Both pedophilia and hebephilia were associated with an increased likelihood of sexual non-contact recidivism, after controlling for time at risk. Based on their high degree of overlap and similar correlates, the major conclusion of this dissertation is that pedophilia and hebephilia are more similar than they are different. Results are discussed within the context of the debate on whether hebephilia is a mental health disorder and the implications for the assessment and risk management of sexual offenders. 


\section{Acknowledgements}

I would like to thank my supervisors Drs. Michael Seto and Alasdair Goodwill for their support and guidance throughout my years as a graduate student. You have both provided excellent research supervision, but more importantly incredible mentorship. I have been very fortunate to have you both as supervisors and very much appreciate all of the opportunities that you have both provided me with whether they were additional research opportunities, financial support to attend conferences and workshops, or the countless hours spent providing me with feedback on my work. I have always valued your advice and I know it will continue to be of great benefit as I move forward with my career. I look forward to our continued work together.

I would also like to acknowledge my dissertation committee who has been incredibly helpful throughout this process. Dr. Todd Girard I very much appreciate your feedback and comments on my work whether it was on my dissertation, course work, or Master's thesis. I appreciate all of the helpful feedback on earlier versions of my dissertations, particularly around additional statistical considerations. Thank you to Dr. James Cantor who was instrumental in helping me access the criminal record data, provided me with access to pre-existing databases, supported my attendance at conferences, allowed me to learn about conducting research in clinical settings, and contributed to my growth as a clinician through clinical practicum opportunities. I have thoroughly enjoyed working with both of you throughout the years and appreciate the time you spent mentoring me and helping me develop into an autonomous researcher. I would also like to thank my dissertation examination committee: Dr. Sara Thompson, Dr. John Turtle, and Dr. Jean Proulx, who served as the external for my defence. I appreciate your thoughtful comments and questions during my examination. 
I would also like to acknowledge the support that my friends, family, and partner have provided throughout the years. You've all provided me with the support, balance, and encouragement necessary to complete my graduate training. I have been blessed to have such an incredible network that is so invested in my success and who have inspired me through their own successes.

Finally, to the provincial government who provided me with financial support through the Ontario Graduate Scholarship and the Social Science and Humanities Research Council who awarded me a Joseph-Armand Bombardier Canada Graduate Scholarship. In addition, I very much appreciate the pre-doctoral research funding awarded by the Association for Treatment of Sexual Abusers (ATSA) who provided the funding necessary to complete my doctoral dissertation. 


\section{Dedication}

I dedicate this work to both of my parents who have always emphasized the importance of education and provided me with the necessary skills to pursue my dreams. I owe my successes to the unwavering support you have both provided throughout the years. 
Author's Declaration....................................................... ii

Abstract............................................................................

Acknowledgements.................................................... v

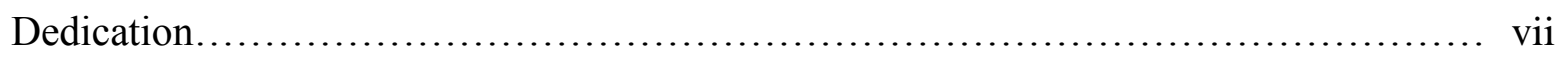

Table of Contents........................................................ viii

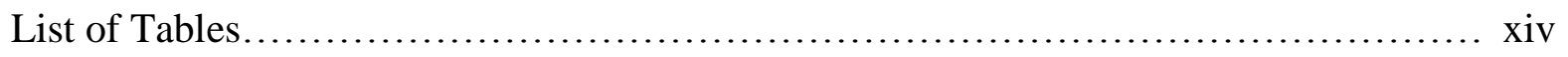

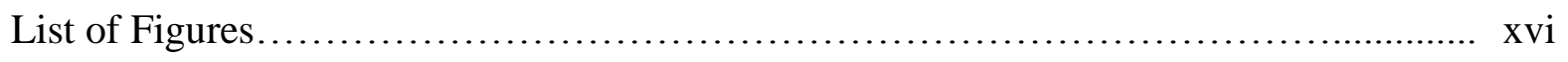

List of Appendices....................................................... xvii

Chapter 1 ........................................................... 2

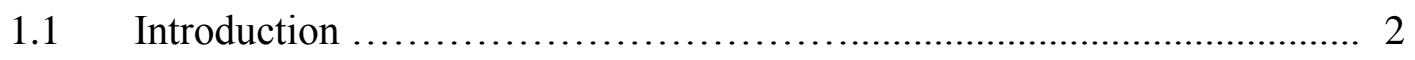

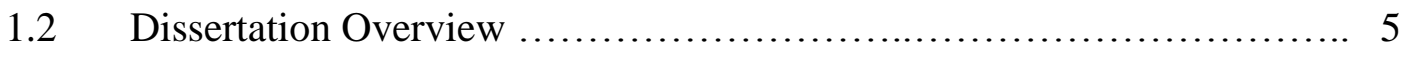

1.3 Literature on Hebephilia as a Sexual Interest ....................... 6

1.4 Pedophilia and Hebephilia in Diagnostic Nosologies .................. 8

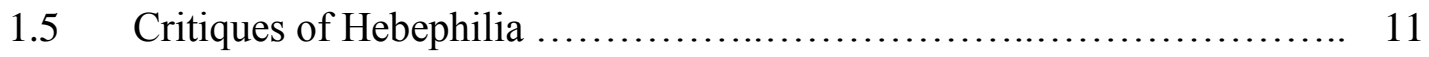

1.5.1 Legal arguments ..................................... 11

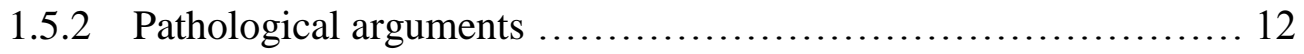

1.6 Significance of the Dissertation ................................. 13

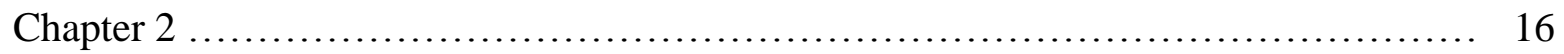

2.1 Study 1: The Utility of Phallometric Assessment in the Detection of Hebephilia: Capturing Patterns of Exclusivity and Non-Exclusivity ....... 16

2.2 Phallometric Assessment...................................... 17

2.3 Phallometric Classification..................................... 18 
$2.4 \quad$ Sexual Response Gradient............................................. 19

2.4.1. Hebephilia and the Sexual Response Gradient ................... 24

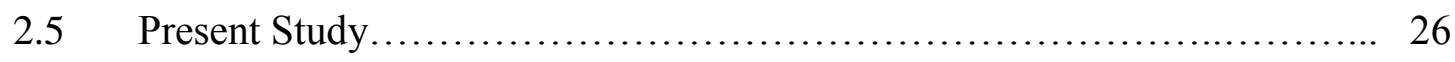

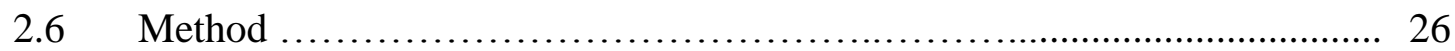

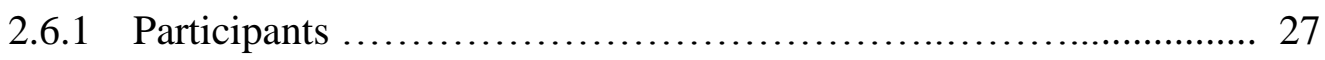

2.6.2 Indicators of Erotic-Age Interest ........................... 28

2.6.2.1. Interview Data .................................... 28

2.6.2.2 Victim Information ...................................... 29

2.6.2.3 Phallometric Assessment ................................... 30

2.6.3 Data Analysis ................................................. 33

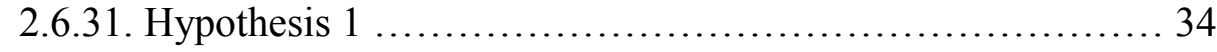

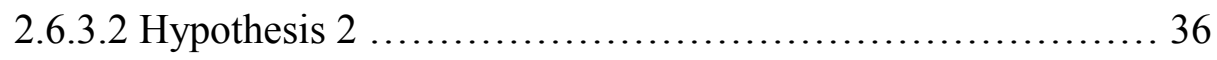

2.6.3.3 Power Analysis ..................................... 36

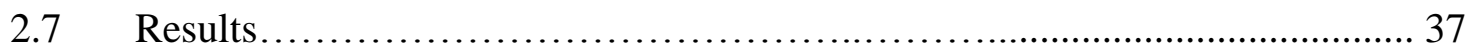

2.7.1 Descriptive Information........................................ 37

2.7.2 Convergent Validity.......................................... 38

2.7.3 Exclusivity of Erotic-Age Interests ................................ 41

2.7.4 Capturing Patterns of Non-Exclusivity ......................... 45

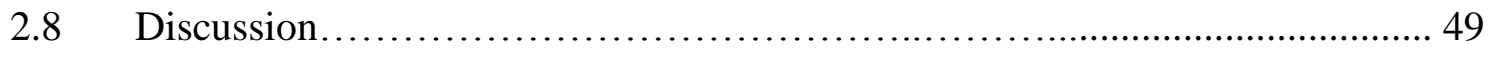

2.8.1 Hebephilia as a Sexual Interest.................................. 50

2.8.2 Overlap between Pedophilia and Hebephilia....................... 51

2.8.3 Implications for the Construct of Hebephilia ........................ 55

2.8.4 Limitations and Future Research Directions ...................... 56 
2.8.5 Summary of Study 1

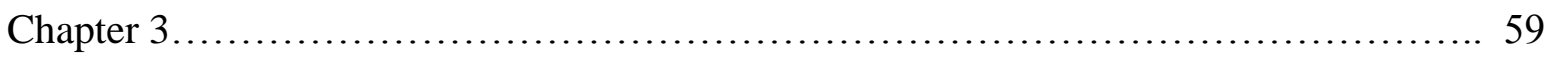

3.1 Study 2: Hebephilia and Victim Age Selection..................... 59

3.2 Hebephilia and Sexual Victim Ages ............................ 56

$3.3 \quad$ Present Study............................................. 61

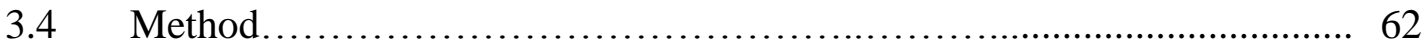

3.4.1 Sample.................................................... 62

3.4.2 Measures .............................................. 62

3.4.3 Procedures................................................... 62

3.4.3.1 Sexual Arousal ..................................... 62

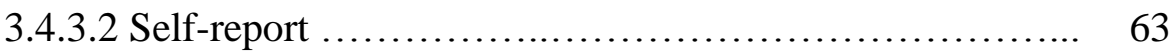

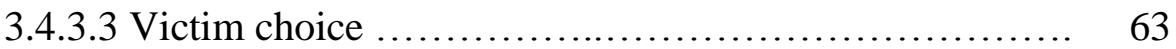

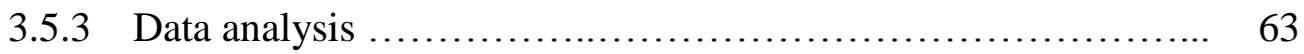

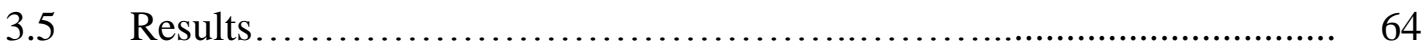

3.5.1 Descriptive Information................................ 64

3.5.2 Erotic-Age Interests and Sexual Victim Choice................ 66

3.5.2.1. Hebephilia .................................... 66

3.5.2.2. Pedophilia .................................... 67

3.5.3 Sexual Victim Age Selection: Impact of Gender Interest ......... 69

3.5.3.1 Hebephilic Sexual Arousal .......................... 69

3.5.3.2 Self-Reported Hebephilic Sexual Interests ............... 71

3.5.3.3 Pedophilic Sexual Arousal ............................ 73

3.5.3.4 Self-Reported Pedophilic Sexual Interests .............. 75 
3.5.4 Victim Age Polymorphism .............................. 77

3.6 Discussion........................................................ 79

3.6.1 Hebephilia and Sexual Offending Victim Age ................. 80

3.6.2 Victim Age Polymorphism............................... 82

3.6.3 Implications......................................... 85

3.6.3.1 Clinical Sexology................................ 85

3.6.3.2 Clinical Forensic Psychology........................ 85

3.6.4 Limitations............................................. 83

3.6.5 Future Research Directions............................... 88

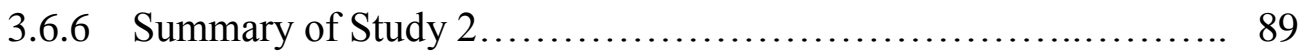

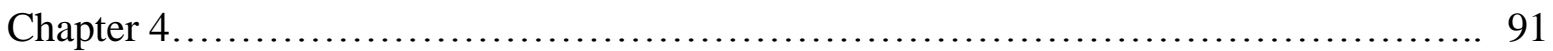

4.1 Study 3: Is Hebephilia Related to Sexual Recidivism among

Identified Adult Male Sex Offenders ............................. 91

4.2 Sexual Interests in Children and Recidivism ........................ 91

4.3 Hebephilia and Recidivism................................... 92

4.4 Present Study................................................. 94

4.5 Method.......................................................... 94

4.5.1 Sample.............................................. 94

4.5.2 Measures .................................................... 96

4.5.2.1 Recidivism .................................... 96

4.5.2.2 Sexual Interests ................................. 96

4.5.2.3 Baseline-risk level ............................. 97

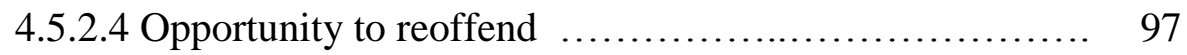




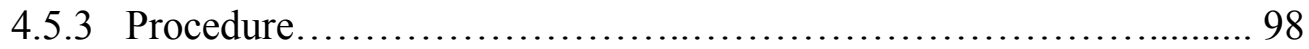

4.5.4 Data analysis ............................................. 100

4.5.4.1 Power Analysis .................................... 100

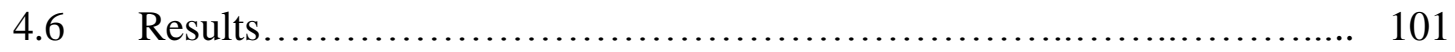

4.6.1 Descriptive Information.................................... 101

4.6.2 Predictive Validity of Erotic-Age Interests..................... 103

4.6.2.1 Sexual arousal ........................................ 103

4.6.2.2 Self-Report ...................................... 104

4.6.2.3 Sexual Behaviour ........................................ 105

4.6.3 Predictive Validity when Taking into Account Street Time ........ 106

4.6.3.1 Hebephilia ......................................... 106

4.6.3.2 Pedophilia ........................................ 107

4.6.3.3 Results by Gender for Hebephilia ....................... 109

4.6.3.4 Results by Gender for Pedophilia ...................... 111

4.6.4 Predictive Validity when Controlling for S99R ................. 113

$4.7 \quad$ Discussion............................................................... 117

4.7.1 Sexual Interest in Children and Recidivism .................. 118

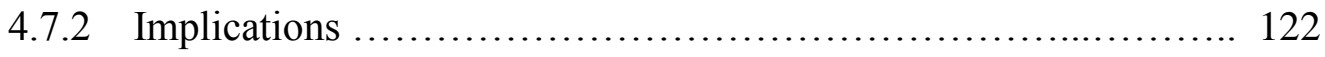

4.7.2.1 Clinical Sexology................................... 122

4.7.2.2 Clinical Forensic Psychology........................... 123

4.7.3 Limitations and Future Research Directions ................... 124

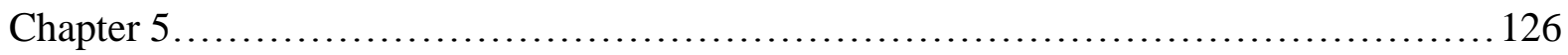




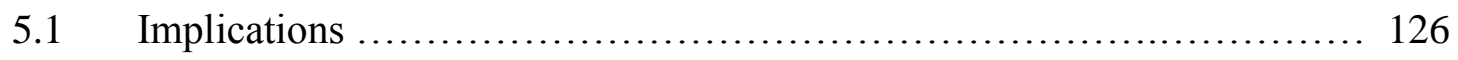

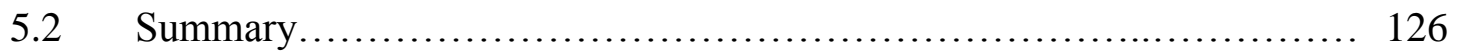

5.3 Implications for Clinical Sexology .................................. 127

5.4 Implications for Clinical Forensic Psychology ............................ 132

5.5 Future Research Direction........................................ 135

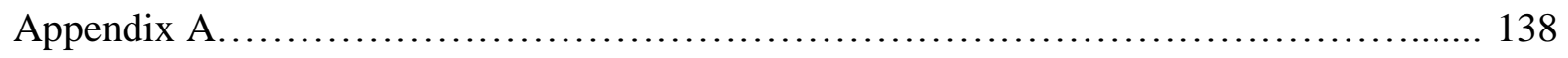

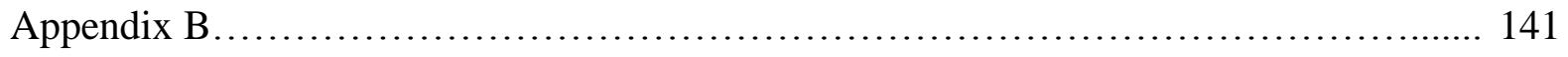

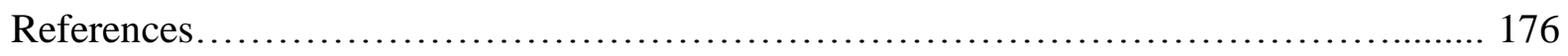




\section{List of Tables}

Table 1. Tanner Stages and Corresponding Erotic-Age Labels ...................... 3

Table 2. Intercorrelations between Indicators of Erotic-Age Interest ................ 39

Table 3. Factor Loadings for the Six Indicators of Erotic-Age Interest................ 41

Table 4. AUCs (SE) for the Prediction of Sexual Arousal Patterns from

Self-Report and Behavioural Indicators of Sexual Interest f...................... 42

Table 5. Phallometric Difference Z scores (SD) for the Full Sample

by Erotic-Age Interest.. ............................................ 46

Table 6. Phallometric Difference Z Scores Separated by Erotic-Gender Interest ........... 48

Table 7. Descriptive Information on Sexual Behaviour ......................... 65

Table 8. Erotic-Age Interest and Sexual Victim Choice .................................... 68

Table 9. Hebephilic Index by Gender and Sexual Victim Choice ........................ 70

Table 10. Self-Reported Hebephilia and Sexual Victim Choice ........................ 72

Table 11. Pedophilic Sexual Arousal and Sexual Victim Choice ........................ 74

Table 12. Self-Reported Pedophilia and Sexual Victim Choice ........................ 74

Table 13. Erotic-Age-Gender Interest and Victim Age Polymorphism................. 76

Table 14. Intraclass Correlations ................................................ 99

Table 15. Descriptive Information....................................... 102

Table 16. AUCs $[95 \%$ CI] for Sexual Arousal and Recidivism........................ 104

Table 17. AUCs $[95 \%$ CI] for Self-Reported Sexual Interest $\ldots \ldots \ldots \ldots \ldots \ldots \ldots \ldots \ldots \ldots \ldots$

Table 18. AUCs $[95 \% \mathrm{CI}]$ for Sexual Behaviour................................ 106

Table 19. Cox Regressions for Hebephilia ..................................... 107

Table 20. Cox regressions for Pedophilia Index............................... 108 
Table 21. Cox Regressions for Hebephilia by Gender .......................... 110

Table 22. Cox regressions for Pedophilia Separated by Gender....................... 112

Table 23. Cox regressions for Hebephilia controlling for S99R .................... 114

Table 24. Cox regressions for Pedophilia Index controlling for S99R.................. 116 


\section{List of Figures}

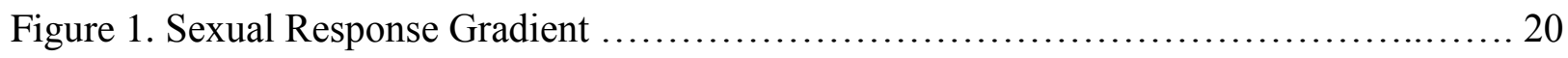

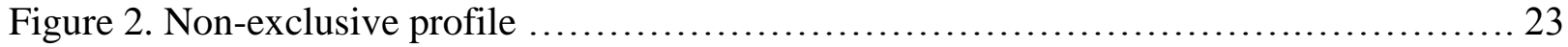

Figure 3. Sexual Arousal (Z Scores) to Different Phallometric Stimuli ......................... 44 


\section{List of Appendices}

Appendix A. Comparison of DSM criteria for Pedophilia............................. 138

Appendix B. CPIC Study Coding Manual............................................. 141 


\section{Chapter 1}

\section{Introduction}

The aim of the dissertation is to provide an in-depth exploration of the role of erotic age interest in sexual offenders, with specific emphasis on hebephilia. Prior to discussing the literature on hebephilia and the study rationales, there will be a brief discussion of the various terminologies that will be used in this dissertation. Hebephilia is a sexual interest, which is an umbrella term that refers to a class of stimuli that an individual would potentially find sexually erotic. There are numerous studies in the literature on sexual interest as it pertains to erotic age (e.g., Beier et al., 2009) and erotic gender (e.g., Ebsworth \& Lalumiére, 2012) interest. These studies have found that sexual interests can be exclusive (e.g., an individual can have a sexual interest only in males) or non-exclusive (e.g., an individual can have a sexual interest for both males and females).

Researchers have used various assessment methods to infer sexual interest. For men, sexual interest can often be inferred from patterns of sexual arousal (i.e., pattern of significant genital response to different sexual stimuli; Chivers, Seto, Lalumière, Laan, \& Grimbos, 2010). Further, sexual interest can also be inferred from self-report and sexual behaviour. From a clinical standpoint, a multi-method assessment of sexual interest is crucial and can add unique variance to the assessment of sexual interest (e.g., Banse, Schmidt, \& Clarbour, 2010), as each of these assessment methods has limitations. For example, during assessments of sexual arousal individuals may attempt to suppress their genital arousal to such a point that the test is invalid.

The focus of the dissertation is on hebephilia, a sexual interest that pertains to erotic-age interest. Hebephilia refers to a sexual interest for pubescent children in Tanner Stage 2 or 3 of sexual development (i.e., early stages of secondary sex development; Blanchard, 2010; 
Blanchard, Lykins, et al., 2009). The Tanner Stages provide a means of quantifying sexual development as it represents a scale of the development of secondary sex characteristics in males and females (Tanner, 1978). These stages are based on pubic hair and breast development in females and pubic hair and genital development in males. It is important to note that for the Tanner Stages, breast and genital development are rated separately and do not always correspond. The stages include corresponding age bands that provide rough estimations of sexual development and range from Stage 1 (pre-pubertal) to Stage 5 (sexually mature; Tanner, 1978). Table 1 presents the Tanner Stages alongside a brief description of the secondary sex characteristics, and the corresponding erotic age interest label (Blanchard, 2010; Blanchard, Lykins, et al., 2009; Hames \& Blanchard, 2012; Tanner, 1978).

Table 1.

Tanner Stages and Corresponding Erotic-Age Labels

\begin{tabular}{|c|c|c|c|}
\hline Tanner Stage & Brief Description & Age range & Preference label \\
\hline Tanner stage 1 & $\begin{array}{l}\text { No secondary sex } \\
\text { characteristics have } \\
\text { developed }\end{array}$ & $\begin{array}{l}\text { Under } 11 \text { (prepubescent } \\
\text { children) }\end{array}$ & Pedophilia \\
\hline Tanner stage 2 & $\begin{array}{l}\text { Breast buds develop and } \\
\text { areola begins to widen. } \\
\text { Male genitalia change. } \\
\text { Small amount of pubic } \\
\text { hair growth. }\end{array}$ & 11 (pubescent children) & Hebephilia \\
\hline Tanner stage 3 & $\begin{array}{l}\text { Male genitalia continue to } \\
\text { change and the penis }\end{array}$ & $\begin{array}{l}12 \text { to } 14 \text { (pubescent } \\
\text { children) }\end{array}$ & Hebephilia \\
\hline
\end{tabular}




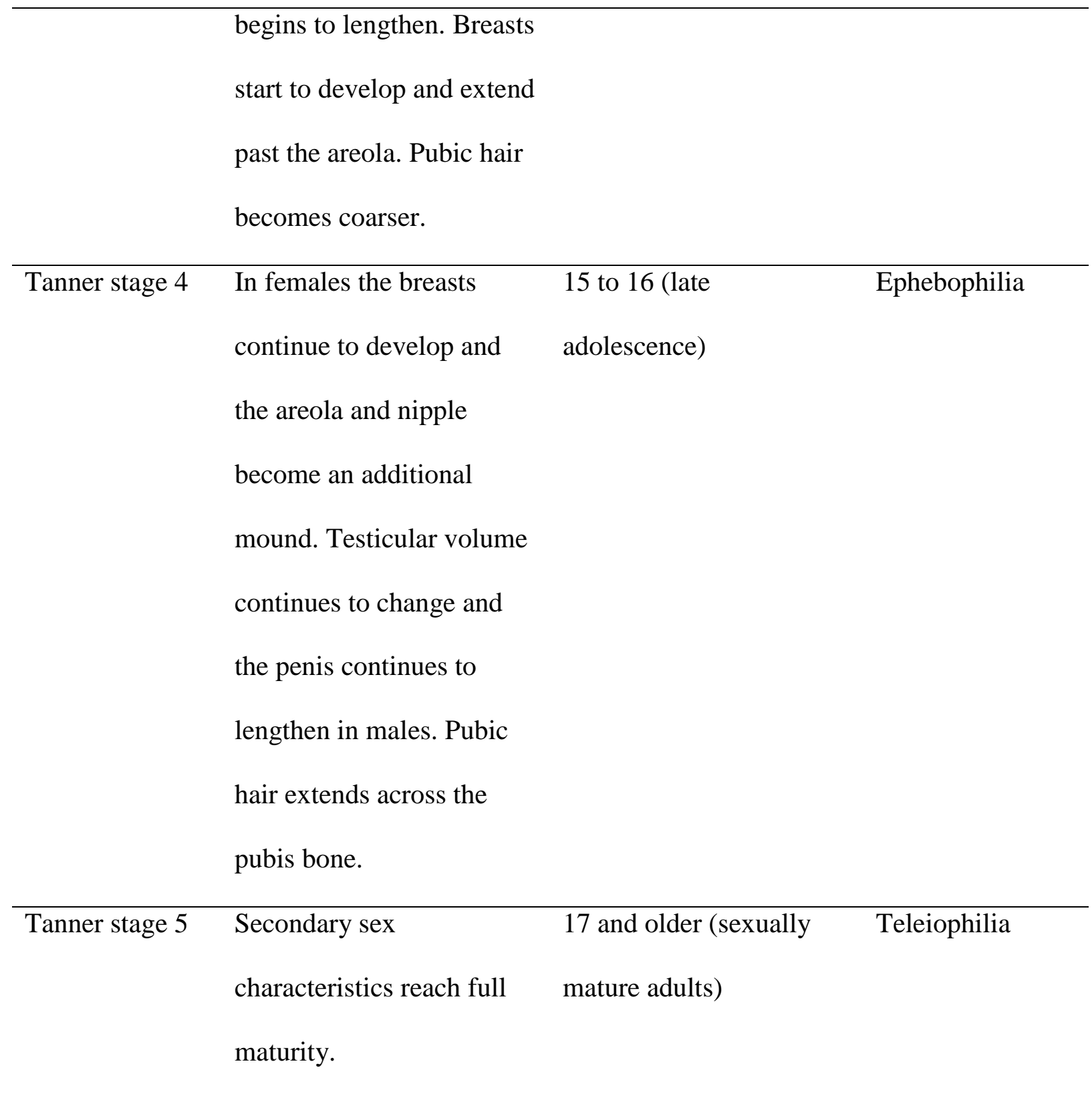

Note. The changes regarding secondary sex characteristics are briefly described, and interested readers should consult Tanner (1978) to receive a full description of each stage. Additionally, the terms child and adult are not based on legal criteria.

As described in Table 1, hebephilia is distinct from pedophilia, which represents a sexual interest in prepubescent children in Tanner stage 1 of sexual development (i.e., no secondary sex characteristic development), and teleiophilia, which is the most typical sexual preference and 
represents a sexual interest in fully mature adults (Hames \& Blanchard, 2012). Both pedophilia and hebephilia are atypical sexual interests, whereas teleiophilia is the species-typical sexual interest (Hames \& Blanchard, 2012; Seto, 2002, 2010).

Hebephilia is often conflated with a sexual interest in typically older adolescents in Tanner Stage 4, which is termed ephebophilia; however, with hebephilia, the focus of the interest is physically immature children who show minimal secondary sex characteristic development. The focus of hebephilic sexual interest is in contrast with ephebophiles who are interested in older adolescents who may appear sexually mature, even if they are under the legal age of consent for sexual activity. Given the level of sexual development in adolescents, ephebophilia is not necessarily uncommon or anomalous (e.g., Hames \& Blanchard, 2012).

\section{Dissertation Overview}

This dissertation is comprised of three studies, focused on establishing the validity of hebephilia as a construct. Study 1 (see chapter 2) will assess the convergent validity of hebephilia in a large sample $(n=2,238)$ of sexual offenders referred to a large Sexual Behaviours Clinic for a comprehensive assessment of their sexual interests. Specifically, $\underline{\text { Study } 1}$ will examine the convergence of various measures of hebephilic sexual interest and will examine the exclusivity of hebephilic sexual interest in a clinical sample.

Hebephilia is arguably most pertinent to furthering our understanding of sexual offending against children, as one could not act on this sexual interest legally without committing a sexual offence against a child. Despite the significant amount of research on the role of pedophilia in sexual offending (e.g., Seto, 2008), there is virtually no research on the role of hebephilia in sexual offending. As such, it is unclear if findings on pedophilia and sexual offending would

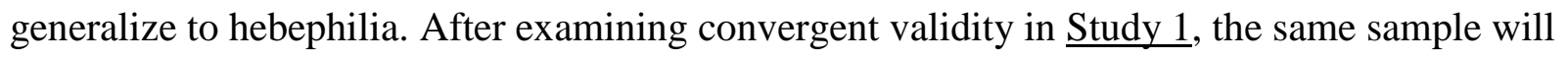




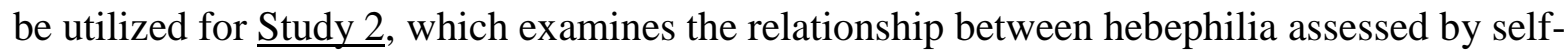
report and patterns of sexual arousal and sexual offending victim choice. Study 2 will assess concurrent validity by examining whether hebephilia corresponds to sexual offending victim age choice, specific to young adolescent victims. Finally, Study 3 (see Chapter 4) will utilize a subsample from $\underline{\text { Study } 1}$ and $\underline{\text { Study } 2}(n=656)$ whose criminal records were coded and analyzed to allow for an examination of the predictive validity of hebephilia over a ten-year follow-up period.

Prior to presenting the results of the studies, a brief overview of the literature on hebephilia is provided in order to provide a broader context on the subject. This is followed by a general discussion on the debate surrounding hebephilia and the way this debate influenced revisions to the diagnostic manual used by clinicians to diagnose mental health disorders. After the introductory chapter, the three empirical studies on hebephilia (chapters 2 through 4) will be presented and followed by a concluding chapter (see chapter 5). Chapter 5 will explicitly discuss the implications of the dissertation for the field of clinical sexology and clinical forensic psychology.

\section{Literature on Hebephilia as a Sexual Interest}

There has been a sizeable literature on hebephilia since the term was first introduced by Glueck in 1955, with the majority of research focused on assessment and etiology. Nonetheless, a significant issue in the literature is that researchers have varied in the way hebephilia is operationalized. For example, hebephilia has sometimes been equated to sexual offending against adolescents (e.g., Baxter, Marshall, Barbaree, Davidson, \& Malcolm, 1984; BrouilleteeAlarie \& Proulx, 2014), while in other studies, it has been referred to as a sexual interest in 
pubescent children (e.g., Blanchard, Lykins, et al., 2009). Different operational definitions have led to significant confusion in the field regarding the construct of hebephilia.

In comparison to the literature that has defined hebephilia as sexual offending against adolescents, there is limited research on hebephilia defined as a sexual interest (as it is operationalized in this dissertation). This is problematic as sexual behaviour does not perfectly correspond to sexual interest (Seto, 2008). For example, many incest offenders do not have pedophilia, even though they have victimized children (e.g., Seto, Kuban, Barbaree, 1999; Seto, 2008). This results in the belief that behaviour must be synonymous with the sexual interest, as many have difficulty understanding why someone would have sexual contact with a child if it did not in some way correspond to their sexual interests.

The majority of research on hebephilia, operationalized as a sexual interest, has focused on the etiology of both pedophilia and hebephilia (e.g., Blanchard, Lykins, et al., 2002; Cantor \& Blanchard, 2012; Cantor et al., 2004, 2005, 2006, 2008, 2012). Interestingly, in clinical populations there tends to be a greater proportion of hebephiles than pedophiles (e.g., Studer, Aylwin, Clelland, Reddon, \& Frenzel, 2002), suggesting it is the more common atypical sexual interest. Further, researchers have tended to find that hebephilic men are intermediate between pedophiles and teleiophiles on a number of variables of interest (e.g., intelligence; Cantor et al., 2004). This finding could lead some to argue that instead of being a distinct group, hebephiles represent a mix of pedophiles who respond to older children and ephebophiles (or teleiophiles) who respond to pubescent children.

A number of studies have also examined the assessment of hebephilic sexual interests. In a recent paper, Blanchard, Lykins, and colleagues (2009) examined the correspondence between self-reported hebephilia and phallometrically assessed sexual arousal to pubescent children. 
Penile plethysmography (referred to as phallometry from this point forward) assesses sexual arousal by monitoring the change in penile blood flow in response to potentially erotic visual and/or audiotaped stimuli (Laws, 2009). In their study, Blanchard, Lykins, and colleagues found support for hebephilia as a distinct erotic age interest. As expected, those men who admitted to sexual interest in pubescent girls had the greatest level of sexual arousal to pubescent girls. Interestingly, those who admitted to sexual interest in pubescent boys had similar patterns of sexual arousal to pubescent and prepubescent boys. This suggests that the ability to detect hebephilia may differ depending on whether the individual is primarily attracted to pubescent boys or girls. Further, it has been found that hebephiles were less likely to have exclusive interested in pubescent children, compared with pedophiles and teleiophiles (e.g., Beier et al., 2013).

\section{Pedophilia and Hebephilia in Diagnostic Nosologies}

Based on their findings and previous research, Blanchard, Lykins, and colleagues (2009) argued that pedophilia should be expanded to include hebephilia in revisions of the Diagnostic and Statistical Manual of Mental Disorders (DSM; Blanchard, Lykins, et al., 2009; Blanchard, 2010). Prior to the release of $D S M-5$ in 2013, hebephilia could be diagnosed under the less precise Paraphilia Not Otherwise Specified. Despite the recommendation by Blanchard, Lykins, and colleagues (2009), the American Psychiatric Association (APA, 2013) decided not to expand pedophilia to include hebephilia. Nonetheless, Blanchard, Lykins, and colleagues' research provided some compelling, albeit limited, evidence of the possible distinctiveness of hebephilia. As such, it is crucial to briefly discuss the existing diagnostic criteria and consider the debate surrounding whether or not to the diagnosis of pedophilia to include hebephilia. It is important to note that regardless of how hebephilia is classified in the DSM, hebephilia is arguably a 
worthwhile construct to study with implications for the field of clinical sexology and clinical forensic psychology.

In the recent revision of the DSM, the APA (2013, p. 697) defines pedophilia as "intense sexually arousing fantasies, sexual urges, or behaviours involving sexual activity with a prepubescent child or children (generally age 13 or younger)." The only difference between pedophilia as a diagnosis in the previous diagnostic manual (i.e., DSM-IV-TR; American Psychiatric Association, 2000) and the revision is that a distinction is made between pedophilia and Pedophilic Disorder in order to avoid pathologizing sexual interests in and of themselves (see Appendix A for DSM-IV-TR criteria, the proposed criteria for DSM-5 in 2012, and the current diagnostic criteria in $D S M-5)$. A person is said to be pedophilic, but not suffering from a disorder, if their sexual interests do not cause functional impairment or distress. Thus, their sexual interest is viewed as anomalous but not psychopathological. This is in contrast to Pedophilic Disorder that can only be diagnosed if the sexual interest results in impairment (e.g., by being arrested for a sexual crime against a child) or distress (e.g., difficulty establishing and maintaining romantic relationships). Therefore, it is distress or impairment that represents the threshold on whether an anomalous sexual interest is psychopathological and in need of clinical attention.

There are also a number of specifiers included alongside the diagnosis to indicate whether the individual is primarily attracted to girls or boys, whether the attraction is exclusive to incest, or if the individual is exclusively interested in children (see Appendix A for specific criteria). According to the DSM-5, one could still be diagnosed with Pedophilic Disorder if sexual interest in children was intense, even if they had some sexual interest in adults. There is no phrasing provided in $D S M-5$ that explains the way in which intensity is characterized. 
Therefore, under the current diagnostic nosology one could be diagnosed as pedophilic if they had exclusive or non-exclusive sexual interest in prepubescent children and exclusivity is also included as a specifier.

The current diagnostic criteria are not in line with the recommendation of Blanchard, Lykins, and colleagues (2009), who discussed a number of ways hebephilia could be included in the DSM. The idea that gained the most traction was that a specifier would be included that reflected the focus of the individual's erotic-age interest, which could be towards a prepubescent child (pedophilic type), pubescent child (hebephilic type) or both prepubescent and pubescent children (pedohebephilic type). The suggestion of Blanchard and his colleagues were incorporated into the proposed revisions for $D S M-5$ (see Appendix A); however, when the DSM-5 was released in May 2013 hebephilia was not included alongside pedophilia, largely due to the controversy over whether it constituted a mental health disorder (see below).

Despite its exclusion in DSM-5, clinicians can diagnose hebephilia with DSM-5 through either the existing, arguably less precise, diagnosis of Pedophilic Disorder (in the DSM-5, sexual interest can be shown for those up to 13 years of age) or Other Specified Paraphilic Disorder. As such, the way hebephilia could be diagnosed in DSM-5 is virtually the same as previous versions of the DSM. Further, the diagnosis of pedophilia in the World Health Organization's International Statistical Classification of Diseases and Related Health Problems (ICD-10) allows clinicians to diagnose pedophilia if the individual being assessed has an erotic age interest in individuals in early pubescence (World Health Organization, 1992), which would correspond to hebephilia. The $I C D$ system is another diagnostic nosology used by many health care professionals, often outside of North America, to diagnose a wide range of health conditions. It is currently under revision and it is unclear whether recent debates in the field of clinical sexology 
will influence the wording of the diagnostic criteria to exclude a distinct diagnosis for hebephilia (i.e., sexual interest to children in early pubescence).

\section{Critiques of Hebephilia}

Since the release of the paper by Blanchard, Lykins, and colleagues (2009) a number of critiques of hebephilia have emerged, with the majority concerned with the conclusion of Blanchard, Lykins, and colleagues (2009) that hebephilia is pathological (Green, 2010; Moser, 2009; Plaud, 2009; Rind \& Yuill, 2012; Tromovitch, 2009; Wakefield, 2012). Some have gone further and argued that hebephilia is not a valid construct (e.g., Franklin, 2009, 2010). The debate regarding the extension of pedophilia to include hebephilia in $D S M-5$ will be summarized, as it provides context to the controversy surrounding the construct, which can only be resolved with additional research.

Legal arguments. Many have criticized hebephilia based on its potential legal implications. Franklin $(2009,2010)$ argued that there exists an invested political interest in the inclusion of hebephilia as a mental disorder as it would increase the number of men subject to civil commitment. Civil commitment involve the incapacitation of sexual offenders deemed "sexually violent predators" after they have served their criminal justice sentence (Elwood, 2009). A diagnosis of a mental health disorder, often a paraphilia, is needed in order to support the designation (Elwood, 2009). In addition, Franklin $(2009,2010)$ argued that the concept of hebephilia only recently emerged in the work of a specific group of authors.

Cantor (2012) provided a response to Franklin $(2009,2010)$ and argued that much of this criticism of hebephilia is without merit. First, there are countless studies on hebephilia from other researchers that predate Blanchard, Lykins, and colleagues' (2009) paper. Secondly, Cantor (2012) countered by arguing that much of the research on hebephilia has been conducted in 
Canada where there are no civil commitment laws. Further, some authors who have criticized hebephilia have invested political and financial motivations when arguing that hebephilia should not be explicitly mentioned in the DSM (Cantor, 2012). Despite these legal arguments, it is important to note that what constitutes a mental disorder should not be swayed by political, legal, or financial interests.

Pathological arguments. In addition to legal arguments, it has been claimed that a diagnosis of hebephilia in $D S M-5$ would pathologize sexual interests that do not conform to societal norms (e.g., Janssen, 2009). Similarly, others have argued that hebephilia is based on a Western notion of the appropriate age of sexual partners, which varies cross-culturally (Green, 2010; Wakefield, 2012). Some have taken this argument a step further by asserting hebephilia is adaptive and has been evolutionarily hardwired across time (Franklin, 2009; Rind \& Yuill, 2012). The common thread in these arguments is that healthy men without psychopathology would be expected to show some level of sexual arousal to pubescent children, and there are some cultures where sexual activity between pubescent children and adults is normative.

The argument that hebephilia is adaptive has been disputed by a number of researchers. Ryniker (2012) argued that anthropological evidence would not support the conclusion that hebephilia is evolutionarily adaptive. In cultures where marriage between pubescent children and adults is common, it is generally a cultural and economic consideration as opposed to an underlying sexual interest in pubescent children. In these cultures, men would not be expected to be sexually aroused to their partners until they reached late adolescence or early adulthood (Ryniker, 2012). Further, in their review of the anthropological literature, Hames and Blanchard (2012) found little data to support the assertion that a sexual relationship occurs immediately when young girls are married to adults; instead marriages in these instances were typically a way 
to preserve a young girl's virginity. They further argued that given the age of these girls when they are married, menses would most likely to not have occurred yet, leaving little to no reproductive benefit. Taken together, these arguments suggest that hebephilia is likely not evolutionarily adaptive.

There is also convincing evidence that men are sexually interested in signs of sexual maturity. Although men are sexually interested in signs of youthfulness, Buss (1994) found that they are also interested in cues of sexual maturity (e.g., a waist to hip ratio of 0.70 , full breasts). As a result, men are most sexually attracted to older adolescent and young adult women (Kenrick \& Keefe, 1996), not pubescent or prepubescent children. Further, pregnancy and child birth in pubescent children (or even adolescents) yields a much higher mortality risk for both the mother and children, in comparison to adult females. These findings also extend to pre-industrial societies where in comparison to older adolescents and young adults who give birth, girls who give birth under the age of 14 have four times the infant mortality rate (Kramer, 2008). Finally, pedophilia and hebephilia are terms reserved for men who have significant levels of sexual interest, relative to their interests in adults. Thus, the argument that hebephilia is adaptive is inaccurate when the term is reserved for those who have a significantly greater sexual interest in pubescent children than adults (Seto, 2010).

\section{Significance of the Dissertation}

This dissertation has important implications for two main areas of study. First, the dissertation will contribute to the field of clinical sexology, specific to the growing body of research on hebephilia as a sexual interest. In essence, it will help to further establish the validity of the construct and potentially influence future revisions of diagnostic manuals used by clinicians to diagnose mental health disorders. Importantly, it will also shed additional light on 
the correspondence of various assessment methods used to assess sexual interest and how this translates into sexual behaviour, which has implications for clinical sexological assessment.

The second field that will be impacted by the dissertation is the field of clinical forensic psychology as it pertains to the assessment, management, and treatment of sexual offenders. Sexual interest in children is a robust predictor of sexual recidivism and has been characterized as a psychologically meaningful risk factor within theoretical models of sexual offending (Hanson \& Morton-Bourgon, 2004, 2005; Mann, Hanson, \& Thornton, 2010; Seto, 2008). In a forensic context, risk to reoffend is crucial as it is considered in the allocation of treatment resources (e.g., high risk sexual offenders should receive intensive services) and informs the selection of treatment targets. These are known as the risk and need principle respectively and these principles contribute to the risk-need-responsivity (RNR) model that is used to guide offender management (Andrews, Bonta, \& Hoge, 1990; Andrews \& Bonta, 2010). Understanding the way hebephilia translates into sexual offending and recidivism is crucial in the provision of risk assessment, which has implications for improving treatment with this population. Hanson, Bourgon, Helmus, and Hodgson (2009) found that programs that used risk assessment to inform treatment were associated with significantly lower recidivism rates than programs that did not adhere to these principles. As such, if hebephilic interest has an important role in sexual offending and recidivism, it must be targeted in an effective manner through appropriate treatment in order to reduce recidivism. Thus, the present studies have significant implications in both the assessment and treatment of sexual offenders.

In summary, Study 1 (chapter 2) will investigate the correspondence between different methods that assess the exclusivity of hebephilic sexual interest in a sexual offender population. $\underline{\text { Study } 2}$ (chapter 3 ) and Study 3 (chapter 4) will directly examine the clinical forensic 
implications of a hebephilic sexual interest by examining how this influences victim selection and the role it plays in the prediction of sexual recidivism. These studies will be followed by a general discussion (chapter 5) that ties the three studies together and discusses implications for the fields of clinical sexology and clinical forensic psychology. 


\section{Chapter 2}

\section{Study 1: The utility of phallometric assessment in the detection of hebephilia: Capturing patterns of exclusivity and non-exclusivity}

Offenders with a sexual interest in prepubescent (pedophilia) or pubescent (hebephilia) children are often less than forthcoming during the assessment of sexual interest, as admitting a sexual interest in children could result in a number of negative consequences (i.e., harsher criminal sentences, greater monitoring, and increased stigma). There are a small proportion of offenders who will admit to sexual interest in children matching their arousal patterns to children (Laws, Hanson, Osborn, \& Greenbaum, 2000; Murphy \& Barbaree, 1994); however, they are the minority. Thus, it could be argued that many offenders with pedophilic or hebephilic interest would attempt to feign sexual interest in sexually mature individuals, due to negative consequences associated with admissions of pedophilic or hebephilic interest.

As such, the assessment of sexual interest among sexual offenders is inherently complicated as offenders are motivated to be portrayed in a positive light. Therefore, there is a need for the use of additional measures of sexual interest that are not subject to self-report bias. One potential indirect measure of sexual interest is sexual behaviour, specific to whether the offender has child victims (Seto, 2008). This is consistent with research that has found that the higher the number of child victims the more likely one is to have a sexual interest in children (Blanchard, Klassen, Dickey, Kuban, \& Blak, 2001; Freund, 1991). Even though having a greater number of child victims may be a good indicator of sexual interest, it is important to remember that having a child victim is not necessarily synonymous with having a sexual interest in children (e.g., Schmidt, Mokros, \& Banse, 2013). For example, Seto (2008) found that only 50 to $60 \%$ of child molesters (i.e., offenders with child victims) were pedophilic. As such, 
researchers have created the Screening Scale for Pedophilic Interests (SSPI; Seto \& Lalumiére, 2001), which was recently revised (SSPI-2; Seto, Stephens, Lalumiére, \& Cantor, 2015). The SSPI considers sexual victim characteristics (e.g., boy victim) that when combined into a scale, are associated with an increased likelihood of pedophilia. Scoring the SSPI requires that those conducting the assessment have comprehensive victim information. Given the large dark figure of sexual crime, it is likely that information on sexual behaviour would be incomplete.

\section{Phallometric Assessment}

Limitations inherent in the assessment of sexual interest have led to the creation of more objective measures of sexual arousal. One such measure is phallometric assessment, a psychophysiological measure of sexual arousal in men developed by Kurt Freund in the late 1950s (Freund, 1963, 1991; Freund, Diamant, \& Pinkava, 1958). Although not originally used for sexual offender assessment, Freund eventually studied its utility in diagnosing pedophilia and hebephilia (Freund, 1965, 1991). It is of note that in his early writings, Freund distinguished between pedophilic and hebephilic interest and included corresponding stimuli (i.e., both prepubescent and pubescent child stimuli were used early on; Freund et al., 1979).

Although phallometry has been viewed as a more objective measure of sexual interest (Laws, 2009), it also has limitations, just like other measures of sexual interest. For example, even though it is harder to fake sexual interest with phallometry, it is not impossible (e.g., Freund et al., 1979; Quinsey \& Bergersen, 1976), and it is easier for men to feign interest in nonpreferred age stimuli than it is to simulate interest in non-preferred gender stimuli (Freund, Watson, \& Rienzo, 1988). Further, Singer (1984) indicated sexual arousal is multifaceted and phallometric assessment only assesses one aspect of sexual arousal, genital responding (e.g., Ackerman \& Beech, 2012; Merdian \& Jones, 2011). Nevertheless, there are currently no strong 
competitive alternatives that provide a more objective measure of sexual interest. As such, the remainder of the review will examine the history of phallometric assessment and issues inherent in the assessment, as it applies to interpretation.

Phallometry measures sexual arousal as change in penile circumference (circumferential assessment, measured in millimeters) or penile blood volume (volumetric assessment, measured in milliliters) to erotic stimuli. Freund developed phallometric assessment as a volumetric measure (see Freund, 1991, for a review of the development of phallometry). Kuban, Barbaree, and Blanchard (1999) demonstrated that volumetric assessment is a more sensitive assessment of sexual arousal as it directly measures total penile blood change, whereas circumferential assessment assesses only one aspect of penile response (i.e., circumference change). In a sample of community volunteers, volumetric assessment was more accurate in comparison to circumferential assessment in predicting group membership at low levels of sexual arousal. Nonetheless, at about $10 \%$ of a full erection (circumferential) or $2.5 \mathrm{ml}$ in penile blood volume change (volumetric), the two were highly correlated (Kuban et al., 1999). It is of note that volumetric phallometry is used in the present dissertation as a measure of sexual interest.

\section{Phallomteric Classification}

Despite its more objective nature, phallometric assessment is not standardized across labs, contributing to debates regarding its clinical utility (e.g., O’Donohue \& Letourneau, 1992; Fedoroff, Kuban, \& Bradford, 2009). One issue with phallometric assessment is that many treatment programs do not have access to it (McGrath, Cumming, Burchard, Zeoli, \& Ellerby, 2010). Further, Howes (1995) conducted a study of phallometric labs across North America and found inconsistency in the ways that different labs displayed stimuli, how the assessment was conducted, and the way phallometric results were interpreted. Most pertinent to the present study 
is the finding of cross-lab variability in the cut-off scores and subsequent interpretation used to classify sexual offenders as pedophilic or hebephilic.

In relation to interpretation, Blanchard, Kuban, and colleagues (2009) conducted a study comparing the different ways phallometric data could be utilized to assist in the diagnosis of pedophilia. Based on the absolute magnitude method, men would be classified as pedophilic if they had a high response to children, irrespective of their responses to other stimulus categories. In the relative magnitude method, men would be diagnosed as pedophilic if their greatest arousal was to children, which acknowledges that men may have other sexual interests as well (i.e., nonexclusivity). Blanchard and colleagues found that relative magnitude was most accurate in classifying those men who had a higher number of child victims. As such, relative assessment based on deviancy indices (i.e., sexual arousal to children, relative to sexual arousal in adults) would be the most valid in determining whether someone has a normative or atypical response profile (see also Lalumière \& Harris, 1998). In this context, an atypical response profile refers to greater sexual arousal to children, relative to adults. This is in contrast to a normative response profile, which refers to greater sexual arousal to adults, relative to children.

\section{Sexual Response Gradient Hypothesis}

Relative assessment is consistent with observations first made by Freund, McKnight, Langevin, and Cibiri (1972) that during phallometric assessment, non-deviant males from the community have some sexual arousal to children. This observation led Freund (1978) to conclude that individuals may have a range of stimuli (organized from most to least) that they find sexually arousing. The finding by Freund (1978) has been referred to as the sexual response gradient (Seto, Laulmiére, \& Kuban, 1999; see Figure 1 for an example). 
Figure 1. Sexual Response Gradient. The figure represents the level of sexual arousal to each of the stimulus categories. The actual responses are for illustrative purposes only and are not based on actual data. The neutral category is comprised of scenery and is used as a control condition. Labels are as follows: Adult $\mathrm{W}=$ adult women stimuli; $\mathrm{Pub} \mathrm{G}=$ pubescent girl stimuli; Prepub G = prepubescent girl stimuli; Prepub B = prepubescent boy stimuli; Pub B = pubescent boy stimuli; and Adult $\mathrm{M}=$ adult men stimuli. The figure represents the classic sexual response gradient for gynephilic (i.e., interested in women) teleiophiles who have a steep gradient and show their greatest response to adult females, followed by pubescent and prepubescent females. The scores for male stimuli are negative, which means that the level of sexual arousal to these stimuli is below their mean level of sexual arousal to all stimuli.

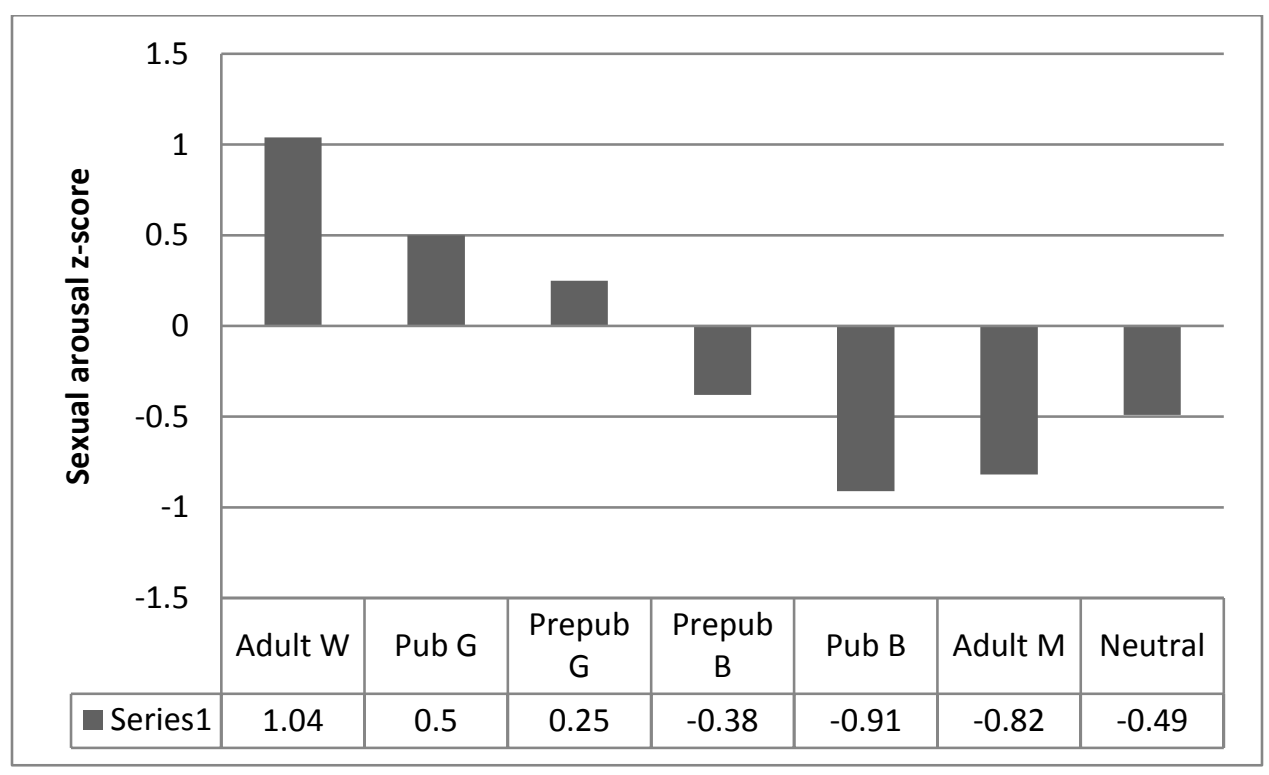

In a recent study, Blanchard and colleagues (2012) examined the sexual response gradient hypothesis in order to examine sexual arousal patterns to non-preferred stimuli. They concluded that sexual interest is based on an overall evaluation of the gestalt (i.e., gender and age) of the criterion stimuli that the person is evaluating is then compared with the individual's most preferred stimuli. This is consistent with the sexual response gradient hypothesis and 
suggests that sexual arousal will decrease the greater the difference between the preferred stimuli and the criterion stimuli. This parallels recent findings from the erotic-age preference literature, as non-offending teleiophiles demonstrated a negative linear relationship between preferred age and sexual arousal (ages included were from young children to adults, not including older adults; Lykins et al., 2010a). Specifically, men who were attracted to adults had the greatest level of arousal to stimuli depicting sexually mature persons, followed by pubescent child stimuli, and then prepubescent child stimuli, with significantly lower levels of arousal to sexual stimuli of the non-preferred gender. A similar pattern has been found in men with sexual offence histories against adult victims (Baxter et al., 1984; Blanchard, Kuban, et al., 2009).

The sexual response gradient hypothesis provides some support for the idea that erotic age interest may be dimensional as opposed to categorical. In support of this assertion, Mackaronis, Strassberg, and Marcus (2011) found that pedophilic interest (assessed via selfreport) was dimensional and should be viewed as a matter of degree as opposed to a categorical distinction. Schmidt and colleagues (2013) reached a different conclusion when they conducted a subsequent taxometric analysis using cognitive measures of pedophilia and found that pedophilia was categorical. One important consideration in evaluating these disparate findings is that Mackaronis and colleagues (2011) used self-report measures of pedophilia, whereas Schmidt and colleagues used implicit cognitive measures of pedophilia. The use of the different measures in these studies may have contributed to the different findings. Further, research on the sexual response gradient hypothesis has been examined exclusively with phallometric data; however, there has been no study that has examined whether phallometric assessment of sexual interest in children is categorical or dimensional. 
The determination of whether erotic-age interest is continuous or categorical (taxon) is an important area of inquiry with significant implications as it would determine whether there is a true class of people with sexual interest in children or whether cut-points are being arbitrarily selected to group individuals for analysis or practical intervention. According to, Rusico, Haslam, and Meron Rusico (2006) determining taxonicity has implications for classification and diagnostics. If erotic-age interest in categorical, then this suggests there would be distinct boundary that separates those with the condition from those without the condition. Thus, there would be relevant cut-off values that could be applied during assessment in order to distinguish those with the condition from those without the condition. Further, it would be possible to establish the prevalence of the taxon if erotic age interest was categorical (Rusico et al., 2006). It is important to note that even if erotic-age interest in continuous this does not mean that cut-off scores could not be applied in order to separate those who score low or high on the variable of interest. Nevertheless, it would challenge the existing diagnostic nomenclature that views individuals with a sexual interest in children as a distinct class. Further, determining whether erotic age interest is a taxon would hold implications for research. Specifically, if it is continuous it suggests that the variable should not be dichotomized in research studies; however, if it is categorical this suggests that dichotomous variables might be more appropriate (for a full discussion of the implications see Rusico et al., 2006).

Despite finding evidence that pedophilia is categorical, Schmidt and colleagues (2013) examined a three class latent class solution and found that there were individuals who exhibited less differentiation between sexual interest to adults and children. This may suggest there is a third taxon of non-exclusive sexual interest, or alternatively could support phallometric research that suggests erotic-age interest is dimensional. Specifically, some men have been found to 
exhibit a non-specific arousal pattern during phallometric testing whereby they respond equally to a variety of distinct stimuli (see Figure 2 for an example; Barbaree \& Marshall, 1989). The non-discriminatory response profile was uncommon in both extrafamilial sexual offenders and men without an offending history (Barbaree \& Marshall, 1989), but common in sexual offenders with victims in different age groups (Michaud \& Proulx, 2009). These findings suggest that classification into exclusive groupings based on sexual arousal could be problematic due to issues of non-exclusivity.

Figure 2. Non-exclusive profile. The figure demonstrates high levels of sexual arousal to pubescent and prepubescent girl stimuli as well as the prepubescent boy stimuli. A negative score means that the level of sexual arousal to that stimulus category is below the patients' mean level of response. This is one example of a non-exclusive profile and the person would be categorized as a pedohebephile, consistent with Blanchard, Lykins, and colleagues (2009) conceptualization of the term. It is of note that there are other response profiles that would be classified as non-exclusive profiles (e.g., similar levels of sexual arousal to pubescent boys and prepubescent boys). The actual responses are for illustrative purposes only and are not based on actual data. The neutral category is comprised of scenery and is used as a control condition. Labels are as follows: Adult $\mathrm{W}=$ adult women stimuli; Pub G = pubescent girl stimuli; Prepub G = prepubescent girl stimuli; Prepub B = prepubescent boy stimuli; Pub B = pubescent boy stimuli; and Adult $\mathrm{M}=$ adult men stimuli. 


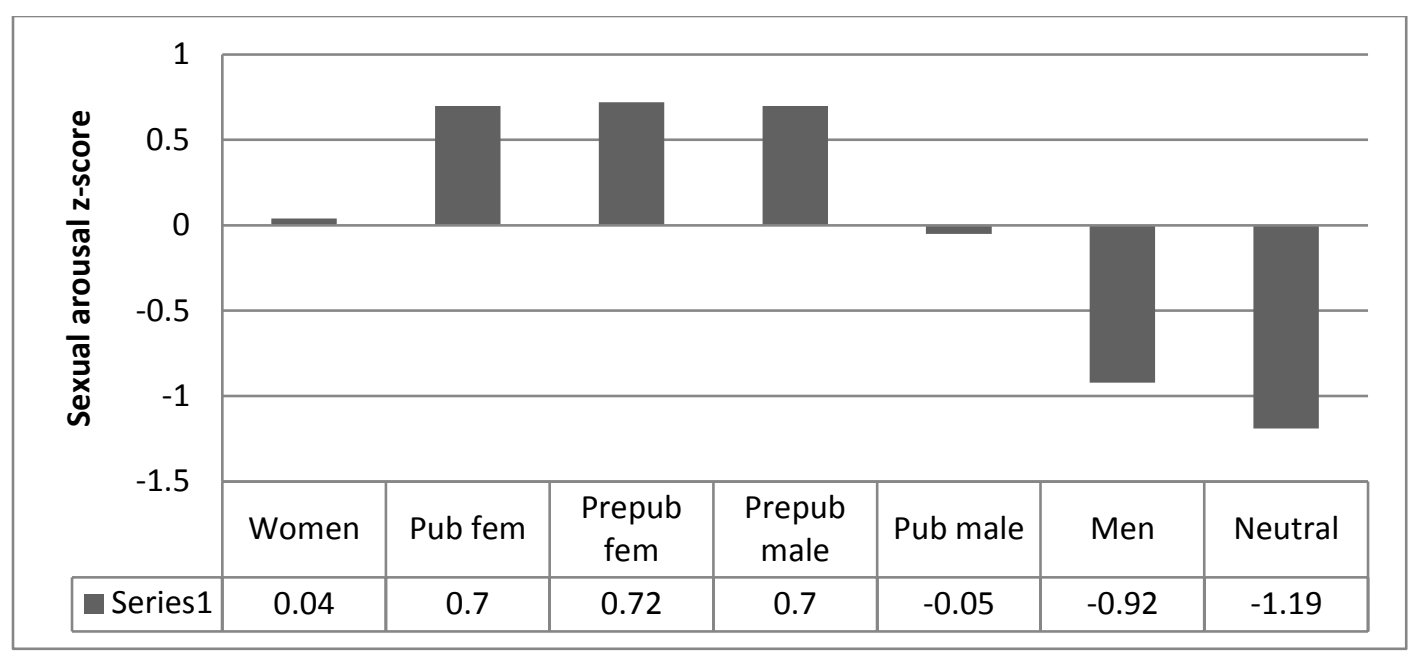

Hebephilia and the sexual response gradient. Research on the sexual response gradient and the dimensionality of erotic-age interest is particularly salient for hebephilia. Based on the sexual response gradient, it would be expected that hebephilic men would have similar levels of sexual arousal to different classes of stimuli, in addition to pubescent children. Specifically, they might also be expected to have high levels of arousal to older adolescents or young adults and/or high levels of arousal to prepubescent children. As such, it could be argued that hebephiles would be less likely to be preferentially attracted to pubescent children (i.e., more likely to show a pattern of non-exclusive sexual interest). Even though sexual non-exclusivity may be more applicable to those with hebephilia, this does not discount the research that suggests there exist men who are preferentially attracted to pubescent children (Beier et al., 2009; Blanchard, Lykins, et al., 2009).

Beier and colleagues (2009) found support for the hypothesis that there is greater nonexclusivity in hebephiles in a community sample of men. Specifically, 58.6\% of self-referred hebephiles (these men self-identified as hebephiles based on an interview) self-reported that they were non-exclusive in their erotic-interest, compared to $38.6 \%$ of pedophiles. Although these figures were not statistically compared by the original authors, subsequent analyses of the data 
from tables provided in the manuscript suggest that hebephiles are more likely to be nonexclusive than pedophiles $\chi^{2}(1)=10.94, p=.001, V=.10$. All of the men classified as teleiophiles were exclusive in their interest towards adults. It was subsequently found in a larger sample, that of those men who identified as hebephilic, $85 \%$ were classified as "mixed types," meaning that only $15 \%$ were exclusively sexually aroused by pubescent children (Beier et al., 2013). As previously discussed, higher rates of non-exclusivity in hebephiles are likely partially explained by the sexual response gradient. Specifically, hebephiles would be expected to have a gradient that is not as steep as pedophiles or teleiophiles who could only move up or down their gradient in one direction.

It is of note that the concept of non-exclusive sexual interest in children is included as a specifier in the $D S M$ criteria for pedophilia. Unfortunately, there is not much detail in the DSM (see Appendix A) on how non-exclusivity is conceptualized or assessed. As such, clinicians have little to no guidance from the diagnostic manual or research in the area on determining whether one is exclusive or non-exclusive. Therefore, individual clinics would have to rely on their own protocols in order to determine the exclusivity of patient's sexual interest in children. At the Centre for Addiction and Mental Health (CAMH) Sexual Behaviour Clinic, (SBC; Toronto, Canada), the location where the individuals in the dissertation were assessed, there is a specific clinic policy to determine non-exclusivity in phallometric results (Freund \& Blanchard, 1989). Specifically, the assessment is weighted in the favour of the client, meaning that if the client has equivalent arousal to adults and children, the client is deemed to have a response profile that is not indicative of a sexual interest in children. Nonetheless, the clinic does classify patients into a non-exclusive pedohebephile group, which means they show significantly greater response to 
children relative to adults, but there is an equivalent level of sexual arousal to prepubescent and pubescent child stimuli.

In their summary of the difficulties inherent in classification of phallometric data, Lalumiére and Harris (1998) highlighted the issue of non-exclusivity, stating that the majority of individuals assessed with phallometry would not exhibit an absolute preference for a certain set of stimuli. Although researchers have examined the most accurate ways to interpret phallometric data and classify men based on exclusive sexual interest (e.g., Harris et al., 1992), there has been limited research in how best to capture the predominantly non-mutually exclusive nature of erotic-age interests. This is an important empirical question that is particularly pertinent to hebephilia based on the above discussion regarding sexual response gradients. Non-exclusivity may be crucial from an offender management perspective, as clinicians are often concerned with best practices associated with managing risk to the community. Non-exclusivity in sexual interests may be particularly relevant from a risk perspective as it may signify that the offender has a greater opportunity to reoffend due to an increased number of potential victims. Further, non-exclusivity may be important in a treatment context as it may be easier to shift the sexual interest of someone who is non-exclusive towards socially appropriate sexual partners.

\section{Present Study}

The present study will expand on the results of Blanchard, Lykins, and colleagues (2009) who found self-reported hebephilic sexual interest was associated with the greatest level of sexual arousal to pubescent children. Blanchard and colleagues only conducted their study with admitters and excluded deniers (i.e., those men who deny a sexual interest in children); the large majority of sexual offenders. As such, the present study will extend these findings by looking at hebephilia in a sample of both admitters and deniers. It will also examine convergent validity 
more specifically, by examining at how phallometric profiles correspond to self-report and sexual behaviour indicative of sexual interest in pubescent children. Further, it will address whether non-exclusivity in phallometric data is more applicable to hebephilia, in comparison to pedophilia and teleiophilia.

There are two main hypotheses that will guide the present study. The first hypothesis is that relative assessment of hebephilic sexual arousal will predict other potential indicators (admitted sexual interests and sexual behaviour, measured by known sexual victims) of hebephilia in a heterogeneous sample of sexual offenders. Thus, there will be evidence for convergent validity. The second hypothesis is that non-exclusivity (e.g., sexual interest in both prepubescent and pubescent children) in phallometric profiles will be more common in hebephiles than in pedophiles or teleiophiles.

\section{Method}

\section{Participants}

The present research was conducted with men referred to the Centre for Addiction and Mental Health's (CAMH) Sexual Behaviours Clinic (SBC) in Toronto, Canada. The majority of patients referred to the SBC have committed non-contact and/or contact sexual offenses involving children; most of the rest have committed sexual offenses involving adults. There are a small proportion of patients who have not committed sexual offenses, but were referred due to problematic sexual behaviours such as an excessive use of pornography or non-criminal paraphilic behaviour that causes distress or relationship problems (e.g., fetishism). The initial sample was comprised of 3,343 men assessed at CAMH between 1995 and 2011. Exclusion 
criteria were as follows: no contact sexual offense victim $(n=582)$, invalid phallometric data ${ }^{1}(n$ $=450)$, and an assessment prior to age $18(n=73)$.

A sample of 2,238 male offenders who admitted to or were convicted of a contact sexual offense were included in the study. In the final sample, $48 \%$ were referred from probation or parole, $17 \%$ were referred by their legal counsel, $28 \%$ were referred by a correctional institution, and $8 \%$ were self-referred. Ethnically, the majority of the sample was Caucasian (79\%), with the remaining 21\% comprised of the following ethnicities: $7 \%$ were Black, $4 \%$ were East Indian, $3 \%$ were Aboriginal, 2\% were Asian, and 2\% were Filipino/Pacific Islander, and 5\% were classified by self-report and interview information in an "other" category. The average age at assessment of the offenders was 39.09 years $(S D=13.22)$. Based on official file information and self-report, the average age at first sexual offense was $29.27(S D=12.94)$ and the average age at last sexual offense was $35.65(S D=12.92)$. Approximately $70 \%$ had been married or been in a common law relationship, and 89\% had no known psychiatric diagnosis at the time of their assessment (not including a paraphilia diagnosis).

\section{Indicators of Erotic-Age Interest}

Interview data. A detailed and standardized Sexual History Interview form has been in use at the SBC since 1995. The form guides a brief clinical interview prior to phallometric testing that is used to gather information on sexual offences, number of consenting sexual partners, admitted sexual partner preferences, information about psychiatric and medical diagnoses, and information about the age of onset of a variety of sexual behaviours (e.g., age at first intercourse).

\footnotetext{
${ }^{1}$ A profile would be invalid if the person's blood volume change was greatest for the neutral stimulus or if blood volume change during phallometric testing was less than $1 \mathrm{cc}$.
} 
Self-reported sexual interest gathered from the interview was treated as a measure of sexual interest. Patients were asked to rank various age and gender categories from 1 (strongest sexual interest) to 5 (no sexual interest). They were able to provide the same ranking for more than one age or gender category (i.e., responses allowed for non-exclusive sexual interest). In the present study, patients were categorized as admitting to a pedophilic or hebephilic sexual interest if they reported moderate to strong sexual interest to prepubescent or pubescent children, respectively. It is important to note that offenders could report equivalent levels of sexual interest to both prepubescent and pubescent children (i.e., these measures were not mutually exclusive). This means that an individual with sexual interest in both prepubescent and pubescent children would be entered into analyses pertaining to both pedophilia and hebephilia. These ratings were also used to categorize offenders based on their self-reported erotic-gender interest (e.g., moderate to strong self-reported sexual interest in females would be indicative of gynephilia, sexual interest in females).

Victim information. Interview and file information was used to group offenders based on their sexual offending history. At the time of the original assessment, the patient's sexual offending history was checked against official records and additional information was collected from the patient (e.g., the number of additional sexual offenses for which he had not been charged). This provided an estimate of the number of offences against children (10 and younger) and offences against young teens ${ }^{2}$ (11 to 14 ) for each offender in the sample. There was also subsequent information on the gender of the victim.

\footnotetext{
${ }^{2}$ The term young teen is used for simplicity to refer to victims between the ages of 11 and 14 throughout the dissertation; however, it is acknowledged that 11 and 12 year olds are technically not teenagers.
} 
The victim information was used as a second potential indicator of pedophilic or hebephilic sexual interests. Specifically, offenders were grouped on whether or not they had three or more child victims or three or more young teen victims. The three or more victim cut-off is significant as it was originally considered as a means to diagnose non-admitters in $D S M-5$ (American Psychiatric Association, 2012). Further, it was used as an empirical criterion by Blanchard et al. (2001) who found the highest sensitivity/specificity of phallometric testing using three child victims as a criterion. They argued that the more child victims (defined as victims under 12) an offender had, the more likely they would be pedophilic. As such, offenders were classified based on offending history. The three or more victim cut-off was used in order to assess how well phallometric results correspond to pedophilia (i.e., three or more child victims) or hebephilia (i.e., three or more young teen victims) as defined by sexual behaviour. Similar to self-report data, hebephiles could also have three or more child victims and pedophiles could have three or more young teen victims (i.e., allowing for non-exclusivity in analyses). This means that an individual with three or more child and young teen victims would be entered into analyses pertaining to both pedophilia and hebephilia.

Phallometric assessment. The SBC clinic utilizes phallometric assessments to assess erotic age and gender preference. Patients were tested with volumetric phallometry and presented with seven categories of stimuli, including a neutral category. The stimuli describe sexual interactions with females and males in the following categories: prepubescent children, pubescent children, and adults. The audiotaped narratives were recorded in second person, present tense and described sexual interactions and the physical attributes (as opposed to the age) of the target person depicted in each stimulus. This ensures that there is no ambiguity in how the offender imagines a child or adult of a specific age. The narratives were read by actors that 
matched the sex of the person in the stimuli, and neutral stimuli were alternately read by men or women. In addition, photographic slides that corresponded to the audiotapes were presented on three adjacent projector screens, which show a nude model in full length (front and rear) and a close-up view of the genital region. Neutral audiotapes were accompanied by slides of scenery (e.g., landscapes).

The patient viewed three slides and each of the trials included three different nude models presented for a total of 18 seconds (total trial duration is 54 seconds). The change in blood flow was recorded four times per second. The test takes approximately one to two hours to complete and involves four blocks of seven trials. The trials were presented in a "fixed pseudorandom order" (Blanchard et al., 2001, p. 121). The interval between these trials varied as it was dependent on the penile blood volume returning to baseline. Lykins et al. (2010b) evaluated the present stimuli used in phallometric testing at $\mathrm{CAMH}$, in comparison with a previous method (one screen with nude models walking towards the camera). It was found that the present method of phallometric testing was the more powerful method of assessment because it generated higher levels of sexual arousal, which means that less of the sample was excluded from interpretation.

The phallometry scoring process conducted at CAMH has been extensively documented by Blanchard and colleagues (2001). Briefly, the patient's greatest level of change from the initial value for each trial is calculated by examining the largest deviation from their baseline and the area under the curve, both of which are converted to standard scores (i.e., the 28 trial scores are converted into 28 standard scores). These 28 standard scores were then averaged across the seven stimulus categories, resulting in seven total category scores. These seven category scores represent the average level of sexual arousal to the different stimuli categories. These milliliter change scores (also referred to as cc) were subsequently transformed to z-scores within 
individuals, which is consistent with the literature that suggests that this is an effective way to reduce variability and ease interpretation (e.g., Harris et al., 1992). Thus, the patient's mean level of responding was categorized as a $\mathrm{z}$-score of 0 . Patients were excluded from analysis of phallometric data if their output index (the average of the three highest responses, regardless of category, and not including the neutral category) was below 1cc, or if their greatest response was to neutral stimuli. Lykins et al. (2010b) established that 1cc of a response is necessary in order to ensure consistency in classification across two different variations of the volumetric phallometry procedure.

Phallometry has shown adequate to moderate reliability whether it is measured by testretest or internal consistency (Harris \& Rice, 1996; Seto, 2008). As there is no standardization across various protocols, it is not possible to provide point estimates of the reliability of phallometric assessment (Harris \& Rice, 1996). Research on the validity of phallometry suggests that there is strong evidence for face validity (Merdian \& Jones, 2011) and some evidence for convergent validity (Laws, 2009). Seto (2001) summarized the research findings on discriminant and predictive validity where there appears to be stronger evidence for these types of validity. Seto (2001) concluded that phallometry discriminated well between subgroups of sexual offenders based on victim history. Further, phallometric assessment has been found to be a robust predictor of recidivism $(d=0.24$; Hanson \& Morton-Bourgon, 2004, 2005), which is likely why it continues to play such a prominent role in the assessment of sexual offenders (Merdian \& Jones, 2011).

In addition to research on psychometric properties of phallometry, the phallometric assessment procedure used at the SBC is standardized and has been the focus of extensive research, adding to its credence. The specificity of the SBC's gender-age preference test for non- 
admitters is $96 \%$, that is, most men who had only ever had sexual contact with women (i.e., no sexual behaviour with children) and who denied any sexual interest in children were not diagnosed as pedophiles. The sensitivity to detect pedophilia was $61 \%$ in those offenders with three or more child victims using a z score cut-off of 0.25 (Blanchard et al., 2001).

A pedophilia and a hebephilia index were created using phallometric data. These indices take into account relative sexual arousal, that is, maximum response to child stimuli relative to maximum response to adult stimuli. For the pedophilia index, the maximum score to prepubescent child stimuli was subtracted from the maximum score to the adult stimuli. For the hebephilia index, the maximum score to pubescent child stimuli was subtracted from the maximum score to adult stimuli. This produced continuous indices where positive scores were indicative of a higher response to children, relative to adults, and negative scores were indicative of higher response to adults, relative to children.

\section{Data Analysis}

Given that the overall goal is to examine phallometric data in relation to hebephilia among admitters and deniers, there were two "gold standards" in the determination of pedophilia and hebephilia. The use of two gold standards is important as it addresses inherent limitations when utilizing one type of assessment of sexual interest (e.g., self-report the individual would need to be truthful). Further, it allowed for a comparison of the findings in order to assess if the different indicators of sexual interest produced similar results. Self-report was used as the first indicator of sexual interest for the sample. As previously discussed, men who admitted moderate to strong pedophilic or hebephilic sexual interest were categorized as such. Further, sexual behaviour was used with those with three or more child victims (i.e., victims under age 11) classified as pedophiles and men with three or more young teen victims (i.e., victims between the 
ages of 11 to 14) classified as hebephilies. As such, sexual behaviour indicators included both those who denied and who admitted to sexual interest in children. It is important to note that for both self-report and victim information, men could self-report or have victims in both categories. For example, men could have three or more child and three or more young teen victims and would be included in analyses pertaining to both pedophilia and hebephilia.

Measures of effect size were considered in the interpretation of all results. Effect sizes are particularly important to consider in the context of the present study, given the large sample size, because even trivial effects could be statistically significant. In the present study, a small effect size was $d=0.20$ or $R^{2}$ or $\eta^{2}=.01$, a medium effect was $d=0.50$ or $R^{2}$ or $\eta^{2}=.06$, and large effect was $d=0.80$ or $R^{2}$ or $\eta^{2}=.14$ (Cohen, 1988; Fritz, Morris, \& Richler, 2012). For correlations, a small effect was $r<.20$, a medium effect was .20 to .30 and a large effect was greater than .30 (Hemphill, 2003). Finally, for AUCs, a small effect was .56, a medium effect was .64, and a large effect was .71 (Rice \& Harris, 2005). Medium to large effects will be the primary focus in the discussion as these effects are more meaningful given the large sample size.

Hypothesis 1. For the first hypothesis, the intercorrelations between the six indicators of sexual interest were examined in order to examine convergent validity. Further, a principal component analysis was used in order to further analyze the convergence of these three indicators. For the intercorrelations and principal component analysis, the indicator variables were left as continuous variables (e.g., the number of child victims). As such, the correlation analyses and PCA included both those who admitted and denied having a sexual interest in children.

The separate indicators of sexual interest were then dichotomized for analyses that examined whether the phallometric indices produce acceptable classification accuracy for the 
prediction of sexual interest or behaviour. Receiver Operator Characteristic (ROC) analyses (Swets, 1988) were utilized as they plot the true positive rate against the false positive rate in order to examine whether classification accuracy is greater than what would be expected based on chance alone. A statistic known as the Area under the Curve (AUC) is produced and ranges from 0 to 1 with a score of .50 representing a chance level of prediction. The AUC can be interpreted as a probability statement (Swets, 1988). For example, in analyses examining whether the hebephilia index predicts hebephilic self-reported sexual interest, an AUC of .67 means that a randomly selected hebephile would have a higher score on the hebephilia index than a randomly selected non-hebephile $67 \%$ of the time.

A categorical variable was also created that combined the self-report and victim information for analyses that examined whether sexual arousal to phallometric stimuli was associated with specific erotic-age interests (i.e., pedophilia, hebephilia, or teleiophilia). This categorical variable was necessary for MANOVA analyses. Offenders were categorized as pedophilic if they had three or more child victims or admitted to a sexual interest in prepubescent children. Similarly, they were classified as hebephilic if they admitted to sexual interest in pubescent children or had three or more young teen victims. Finally, offenders were classified as teleiophilic if they had no child victims and reported sexual interest in adults, while denying sexual interest in children. As such, deniers were operationalized within the context of this variable as those offenders who denied sexual interest in children, but had three or more child victims. Gender interest was also taken into account in analyses, by examining the results for the total sample and then conducting the results for the men who self-reported a sexual interest in females and the men who self-reported a sexual interest in males. This approach was utilized in 
order to examine the overall pattern for the entire sample and then to examine whether results differed by erotic-gender interest.

Hypothesis 2. In order to test the second hypothesis (i.e., hebephiles are more likely to be non-exclusive in sexual interest), the categorical erotic-age interest variable was utilized as a between subject factor in a MANOVA. The dependent variables were a series of absolute difference scores calculated to contrast sexual arousal to the following sets of stimuli: absolute difference between sexual arousal to female adults and pubescent children; absolute difference between sexual arousal to pubescent girls and prepubescent girls; absolute difference between sexual arousal to prepubescent girls and prepubescent boys; absolute difference between sexual arousal to male prepubescent children and pubescent children; absolute difference between sexual arousal to pubescent boys children and adult men.

Power analysis. A power analysis was conducted using $\mathrm{G}^{*}$ Power 3 (Faul, Erdfelder, Lang, \& Buchner, 2007) based on a medium effect size $(r=.34)$ between the Screening Scale for Pedophilic Interest (SSPI; Seto \& Lalumiére, 2001) and phallometrically assessed pedophilic sexual arousal. This was the effect size used to calculate power for the present study, because the SSPI assesses victim information in order to provide an assessment of pedophilic interest and the present study examines behavioural and self-report indicators of hebephilia. Based on this effect size, an estimated 100 offenders would be needed in order to achieve power of .80. As such, the present study was deemed more than adequately powered for analyses. The power analysis for

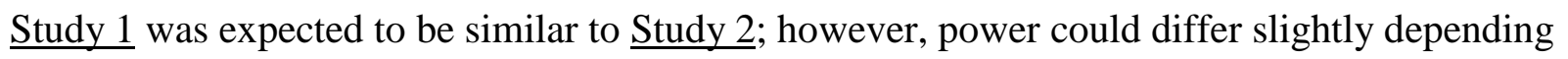
on the types of analyses being conducted. 


\section{Results}

\section{Descriptive Information}

The average score on the hebephilia index was $-0.10(S D=0.86)$ and the average score on the pedophilia index was $-0.46(S D=0.95)$. Skewness and kurtosis were within acceptable ranges for all phallometric category scores and the phallometric indices, $|2|$ and $|7|$, respectively (Fields, 2013). In relation to self-reported sexual interest, 6\% of offenders admitted to pedophilic sexual interest, and $20 \%$ of offenders admitted to hebephilic sexual interest. For sexual behaviour, $13 \%$ had three or more young child victims, and $18 \%$ had three or more young teen victims.

When combining the two measures of sexual interest to develop a categorical variable for the MANOVAs, $32 \%$ were hebephiles, $14 \%$ were pedophiles, $19 \%$ were pedohebephiles (i.e., sexual interest in both prepubescent and pubescent children), and $36 \%$ were teleiophiles. Using this approach, there were 1179 men who were not classified because they had one or two child victims and denied sexual interest in children. As such, their sexual interest was ambiguous and they were excluded from analyses. Additionally, the group of pedohebephiles emerged because the initial sexual interest and sexual behaviour variables that were used to create the categorical variable were not mutually exclusive. They were not retained as their own group as it was expected that they could be further classified into discrete groups based on predominant sexual interest. For these offenders, their self-reported interests were examined, and they were classified based on their strongest reported sexual interest; however, $6 \%$ reported equivalent sexual interest in prepubescent and pubescent children. For the remaining $6 \%$ of pedohebephiles, the greatest number of child or young teen victims was used to classify offenders. For example, in cases of equivalent sexual interest in pubescent and prepubescent children, the offender was classified as a pedophile if he had a greater number of child victims than young teen victims. At this point, 
only $1 \%$ or 11 pedohebephiles were unable to be classified and were excluded from analyses. Therefore, there was a total sample of 1048 for MANOVA analyses: 44\% were hebephiles, $20 \%$ were pedophiles, and $36 \%$ were teleiophiles.

\section{Convergent Validity}

Intercorrelations were examined as a means to assess convergent validity among the various indicators of pedophilia and hebephilia (see Table 2). The correlations provided strong evidence of convergent validity for both pedophilia and hebephilia. Specifically, for hebephilia a medium sized association was observed for self-report and sexual arousal. There was a small but statistically significant effect for the correlation between the sexual arousal and behavioural indicators of hebephilia.

Of significant interest was convergence between the various indicators of pedophilia and hebephilia. In particular, there was a large effect for the correlation between the pedophilia and hebephila indices and for the correlation between pedophilic and hebephilic self-reported sexual interest. Further, different types of indicators of pedophilia and hebephilia were correlated with each other, suggesting that the overlap extends beyond method covariance. For example, the correlation between self-reported hebephilia and the pedophilia index was a medium effect $r=$ .29. Overall, the results suggested that there is considerable convergence between pedophilia and hebephilia, independent of method covariance. 
Table 2.

Intercorrelations between Indicators of Erotic-Age Interest

\begin{tabular}{|c|c|c|c|c|c|}
\hline & Pedophilia & Hebephilia & $\begin{array}{l}\text { Self-report } \\
\text { pedophilia }\end{array}$ & $\begin{array}{l}\text { Self-report } \\
\text { hebephilia }\end{array}$ & $\begin{array}{c}\text { Child } \\
\text { victims }\end{array}$ \\
\hline Pedophilia & - & - & - & - & - \\
\hline \multicolumn{6}{|l|}{ Index } \\
\hline Hebephilia & $.84 * * *$ & - & - & - & - \\
\hline \multicolumn{6}{|l|}{ Index } \\
\hline Self-report & $.31 * * *$ & $.23 * * *$ & - & - & - \\
\hline \multicolumn{6}{|l|}{ pedophilia } \\
\hline Self-report & $.29 * * *$ & $.30 * * *$ & $.66^{* * *}$ & - & - \\
\hline \multicolumn{6}{|l|}{ hebephilia } \\
\hline Child & $.28 * * *$ & $.22 * * *$ & $.47 * * *$ & $.31 * * *$ & - \\
\hline \multicolumn{6}{|l|}{ Victims } \\
\hline Young Teen & $.15^{* * *}$ & $.13 * * *$ & $.22 * * *$ & $.31 * * *$ & $.29 * * *$ \\
\hline
\end{tabular}

Note. Pearson correlations were computed on the continuous variables for each of the indicators. $* * * p<.001$ 
In order to further examine the relationship between the various measures of pedophilia and hebephilia, a principal component analysis was conducted on the six indicators of sexual interest. An oblique rotation (direct oblim) was used as it was expected that the factors would be correlated to some degree. The KMO Measure of Sampling Adequacy was .62, which indicated that the sample size was sufficient for PCA. The Bartlett's Test of Sphericitiy was significant $\chi^{2}$ $(15)=5224.18, p<.001$, the correlations in the R matrix were not an identity matrix, and PCA was appropriate.

Based on Kaiser's criterion of extracting factors with eigenvalues of one and above, two factors were extracted which together explained $66.74 \%$ of the variance. The scree plot was consistent with Kaiser's criterion and justified the retention of two components. Table 3 shows the factor loadings after rotation. Both the behavioural and self-report indicators of pedophilia and hebephilia loaded on factor 1 , whereas both sexual arousal indicators loaded on factor 2 . Factor 1 appears to be a pedophebephilia factor as self-report and sexual behaviour in particular load on to this factor. Factor 2 appears to be a teleiophilia factor best indicated by how a person responds to children, relative to adults during phallometric testing. Furthermore, the PCA suggests that beyond method covariance, there is considerable convergence between pedophilia and hebephilia as both indicators of sexual arousal loaded onto factor 2 ; however, the other indicators of sexual interest also loaded on to this factor to a lesser degree. 
Table 3.

Factor Loadings for the Six Indicators of Erotic-Age Interest

\begin{tabular}{lcc}
\hline Indicators & Factor 1 & Factor 2 \\
\hline Pedophilia index & .33 & $-\mathbf{9 6}$ \\
Hebephilia index & .28 & -.96 \\
Self-report pedophilia & $\mathbf{. 8 4}$ & -.29 \\
Self-report hebephilia & $\mathbf{. 8 1}$ & -.31 \\
Child victims & $\mathbf{. 6 9}$ & -.26 \\
Young teen victims & $\mathbf{. 5 7}$ & -.10 \\
\hline Eigenvalues & 2.71 & 1.30 \\
$\%$ of Variance & $45.17 \%$ & $21.58 \%$ \\
\hline
\end{tabular}

Note. A principal component analysis was conducted on the continuous indicator variables.

Factor loadings above .40 are in boldface.

Exclusivity of Erotic-Age Interests

For both the pedophilia and hebephilia indices, all of the AUCs were significant at the $p$ $<.001$ level (see Table 4). Interestingly, both of the indicators of pedophilia $(Z=5.98, p<.001)$ and hebephilia $(Z=2.88, p=.003)$ had higher classification accuracy when predicting pedophilic sexual interest compared to hebephilic sexual interest. This finding did not generalize to the behavioural indicators of sexual interest (for hebephilia $Z=1.7, p=.087$ and pedophilia $Z=$ $1.65, p=.098)$. Nonetheless, the AUCs were always higher for the indicator that they were expected to predict (e.g., pedophilia index produced a higher AUC than the hebephilia index for the prediction of pedophilic sexual interest), with the exception of $3+$ young teen victims. Even 
though they were higher it is important to note that the differences between the pedophilia and hebphilia indices were not significant.

Despite these findings, both the pedophilia and hebephilia indices predicted all self-report and sexual behaviour variables. The AUCs were in the medium effect size range, with the exception of a small effect for the prediction of $3+$ young teen victims for the hebephilia index. Further, the effects were large for the prediction of pedophilic self-reported sexual interest. Table 4.

AUCs (SE) for the Prediction of Sexual Arousal Patterns from Self-Report and Behavioural Indicators of Sexual Interest

Hebephilia index $\quad$ Pedophilia index $\quad$ Z

\begin{tabular}{llll}
\hline Self-report pedophilia & $.77(.02)$ & $.83(.02)$ & 0.03
\end{tabular}

Self-report hebephilia

$.69(.01)$

$.67(.02)$

0.10

$3+$ child victims

$.66(.02)$

$.69(.02)$

0.02

$3+$ young teen victims

$.61(.02)$

$.64(.02)$

0.02

Note. AUCs were all significant at the $p<.001$ level. The columns represent the continuous predictor variables, whereas the rows represent the dichotomous outcome variables. Z scores 
were computed that compared the AUCs produced for the pedophilia and hebephilia indices. None of the $\mathrm{Z}$ scores were significant.

A MANOVA was conducted in order to examine whether the groups (i.e., groups based on combination of self-reported interest and sexual behaviour) differed on the combination of the sexual arousal to the different phallometric response scores. Using Wilks statistic, there was a significant medium effect, $\Lambda=.77, F(12,2080)=23.67, p<.001, \eta_{p}{ }^{2}=.12$ that suggested the groups differed on the combination of sexual arousal to the various stimulus categories (see Figure 3). All of the follow-up univariate ANOVAS were significant (all $p<.001$, but phallometric response to male adults $p=.001$ ). The effect sizes varied and from small to medium with sexual arousal to adult women producing the largest effect size $\left(\eta_{p}{ }^{2}=.12\right)$, followed by prepubescent girls $\left(\eta_{p}^{2}=.09\right)$, prepubescent boys $\left(\eta_{p}^{2}=.06\right)$, pubescent girls $\left(\eta_{p}^{2}=.04\right)$, pubescent boys $\left(\eta_{p}^{2}=.03\right)$, and adult men $\left(\eta_{p}^{2}=.01\right)$.

Upon further examination, contrasts suggested that the hebephiles had a greater response to pubescent girls than the pedophiles $(p=.015, d=0.19)$ and pedophiles had a greater response to prepubescent boys than the hebephiles $(p=.016, d=-0.18)$; however, both of these effect sizes were small. The teleiophiles and hebephiles differed on all of the stimulus categories. For the difference between the hebephilies and teleiophiles, the effect sizes were medium to large: sexual arousal to adult women $(p<.001, d=-0.75)$, prepubescent girls $(p<.001, d=0.61)$, prepubescent boys $(p<.001, d=0.49)$, pubescent girls $(p<.001, d=0.46)$, and pubescent boys $(p<.001, d=0.35)$, with the exception of sexual arousal to male adults which produced a small effect size $(p=.011, d=-0.13)$. Overall, there were a greater number of significant differences in the large range when comparing the hebephiles with the teleiophiles, than when comparing the hebephiles and pedophiles. 
Figure 3. Sexual Arousal (Z Scores) to Different Phallometric Stimuli. Standard error bars are included on the figure. On the $\mathrm{x}$-axis: Adult $\mathrm{W}=$ Adult Women, $\mathrm{Pub} \mathrm{G}=$ Pubescent Girl, Prepub G= Prepubescent Girl, Prepub B = Prepubescent Boy, Pub = Pubescent Boy, and Adult $\mathrm{M}=$ Adult Men.

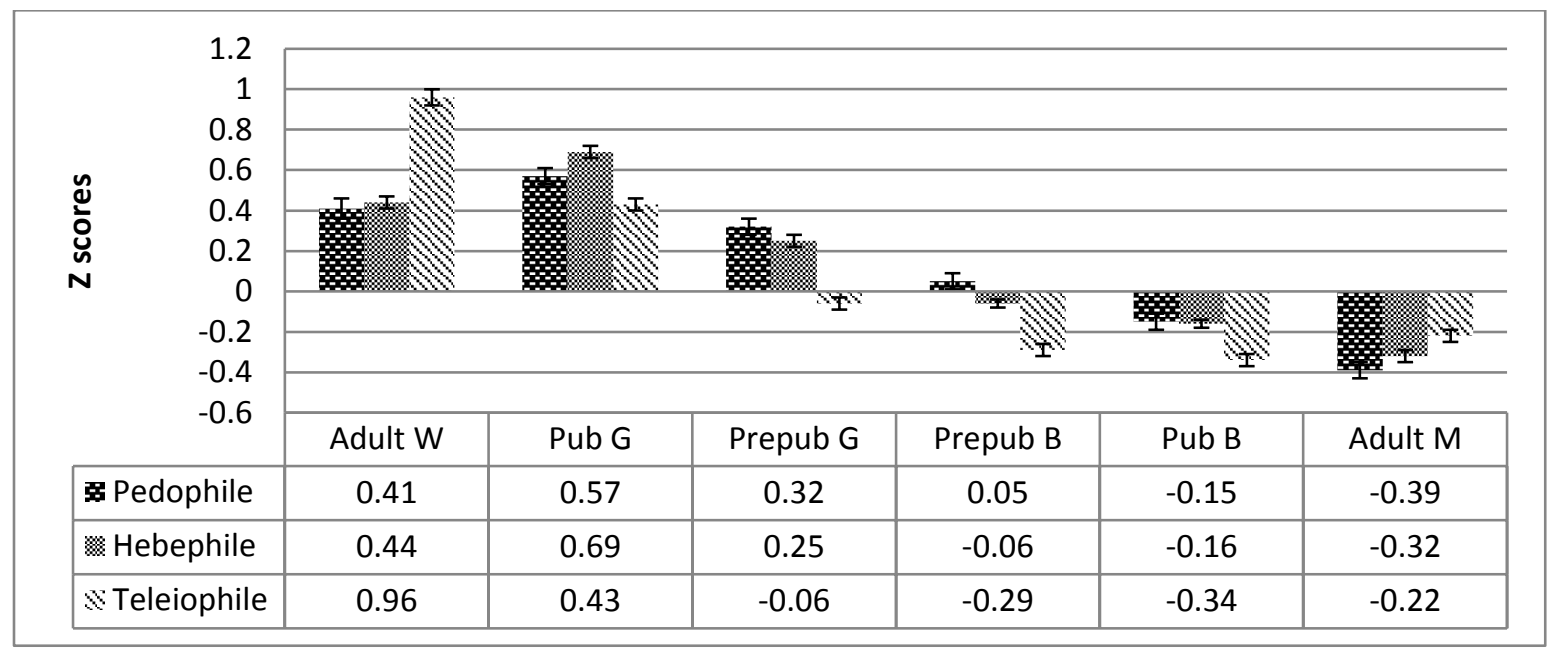

Separate MANOVAS were then conducted for sexual interest in males and sexual interest in females. For these analyses, only the phallometric score that corresponded to the person's gender interest were examined. ${ }^{3}$ For sexual interest in females $(n=975)$, the results suggested that the phallometric scores in combination differentiated the four groups $\Lambda=.82, F(6,1940)=$ $34.43, p<.001, \eta_{\mathrm{p}}{ }^{2}=.10$, producing a medium effect size. Univariate ANOVAs were used to further examine these group differences, and all of the follow-up ANOVAs were significant $(p<$ $.001)$. All effect sizes were medium: sexual arousal to female adults $\left(\eta_{p}{ }^{2}=.12\right)$, prepubescent children $\left(\eta_{p}^{2}=.10\right)$, and pubescent children $\left(\eta_{p}^{2}=.06\right)$. Contrasts suggested that hebephiles had significantly higher levels of sexual arousal to pubescent girls compared to pedophiles, but the effect size was small ( $p=.005, d=0.23)$. Further, compared with the teleiophiles, they had a

\footnotetext{
${ }^{3}$ Gender was not entered as a covariate into analyses as it was not a continuous variable. Further, cell sizes would have been too small for some groups (e.g., there were few offenders who reported a sexual interest in adult men).
} 
higher response to prepubescent $(p<.001, d=0.66)$ and pubescent girls $(p<.001, d=0.57)$, and a lower response to adult women $(p<.001, d=-0.70)$. All of the effect sizes were large when comparing the hebephiles and the teleiophiles.

The sample size was significantly reduced $(n=177)$ for sexual interest in males, because few individuals self-reported a moderate to strong sexual interest in males. Nonetheless, the results suggested there was a significant medium effect, $\Lambda=.76, F(6,344)=8.66, p<.001, \eta_{p}^{2}=$ .13. Univariate effects were in the large range for sexual arousal to prepubescent boys $(p<.001$,

$\left.\eta_{p}{ }^{2}=.14\right)$ and adult men $\left(p<.001, \eta_{p}^{2}=.16\right)$ and in the small range for sexual arousal to pubescent boys $\left(p=.013, \eta_{p}^{2}=.05\right)$. The hebephiles differed significantly from the teleiophiles and large effect sizes were observed on all of the sexual arousal variables. Compared to the teleiophiles, hebephiles had a higher response to prepubescent $(p<.001, d=1.23)$ and pubescent boys $(p<.001, d=0.52)$, and a lower response to adult stimuli $(\mathrm{p}=.020, d=-1.15)$. The only difference between the pedophiles and the hebephiles, was the hebephiles had a higher response to pubescent boys ( $p=.026, d=0.42)$, which was a small effect.

\section{Capturing Patterns of Non-Exclusivity}

For analyses pertaining to non-exclusive sexual interest, a MANOVA was conducted on the absolute difference scores between adjacent stimuli (i.e., the dependent variables). Absolute values were examined because the z-scores could be positive or negative. A large absolute difference score signified greater differentiation between sexual arousal to the two sets of stimuli, suggestive of greater exclusivity in sexual arousal. A small absolute difference score signified less differentiation between responses to the two sets of stimuli, suggestive of less exclusivity in sexual arousal to the two stimulus categories. The absolute difference scores were within acceptable levels for skewness |2| and kurtosis |7| (Fields, 2013). MANOVAs were carried 
out for the full sample (including both male and female interest) and separately by gender interest.

The overall MANOVA (see Table 5) suggested there was a significant group difference on the combination of difference scores, $\Lambda=.96, F(10,2082)=4.88, p<.001, \eta_{p}{ }^{2}=.02$, but the effect size was small. The significant MANOVA was followed by univariate ANOVAs. The absolute difference scores differentiated the groups, but effect sizes were small: arousal to adult women versus pubescent girls $\left(p=.019, \eta_{p}{ }^{2}=.01\right)$, arousal to prepubescent girls versus prepubescent boys $\left(p<.001, \eta_{p}{ }^{2}=.02\right)$, arousal to prepubescent versus pubescent boys $(p=.024$, $\left.\eta_{p}^{2}=.01\right)$ and arousal to pubescent boys versus adult men $\left(p=.008, \eta_{p}^{2}=.01\right)$.

A series of contrasts were used to examine significant group differences. There was only one significant difference between the pedophiles and the hebephiles. Specifically, the hebephiles were less exclusive than the pedophiles in their sexual arousal to pubescent and prepubescent boys $(p=.008, d=-0.25)$. Compared to the teleiophiles, the hebephiles had less exclusivity between adult women and pubescent girls $(p=.017, d=-0.16)$. This is in contrast to the difference score for prepubescent girls versus boys $(p<.001, d=0.34)$ and for adult men and pubescent boys ( $p=.020, d=0.16$ ), where the teleiophiles had less exclusivity compared with the hebephiles. All effect sizes were in the small range.

Table 5 .

Phallometric Difference Z scores (SD) for the Full Sample by Erotic-Age Interest

\begin{tabular}{llll}
\hline Difference Score & Hebephile & Pedophile & Telophile
\end{tabular}

\begin{tabular}{llll}
\hline Adult women versus & $.71(.51)$ & $\mathbf{. 6 8}(.51)$ & $.80(.64)$ \\
pubescent girl & & &
\end{tabular}




\begin{tabular}{llll}
\hline Pubescent versus & $.55(.43)$ & $\mathbf{. 5 1}(.39)$ & $.58(.43)$ \\
prepubescent girl & & & \\
Prepubescent girl & $.71(.50)$ & $.69(.57)$ & $\mathbf{. 5 5}(.43)$ \\
versus boy & & & \\
Prepubescent versus & $\mathbf{. 3 8}(.33)$ & $.46(.38)$ & $.39(.33)$ \\
pubescent boy & & & \\
Pubescent boy versus & $.51(.46)$ & $.55(.45)$ & $\mathbf{. 4 4}(.40)$ \\
adult men & &
\end{tabular}

Note. Numbers of boldface indicate the lowest score for that category, which indicates less differentiation between the two sets of stimuli. The difference scores are based on the differences in $\mathrm{z}$ scores between the stated stimulus categories.

The same analytic approach was then conducted separately by gender interest. This time, however, only the difference scores that corresponded to the gender interest were included. The exception to this was that the difference score for prepubescent boys and girls was included in both sets of analyses, as there is evidence to suggest that pedophiles are more likely to be nonexclusive in their gender interest than others (e.g., Blanchard et al., 2012).

When conducting the MANOVA for sexual interest in males, $A=.87, F(6,344)=4.06$, $p=.001, \eta_{p}{ }^{2}=.07$, results were significant (top panel of Table 6). Follow-up ANOVAs suggested there was a significant difference on the contrast between pubescent versus prepubescent boys $\left(p=.001, \eta_{p}{ }^{2}=.07\right)$, with the hebephiles showing less exclusivity compared with the pedophiles $(p<.001, d=-0.63)$. Further, for the absolute difference score between sexual arousal to pubescent boys and adult men $\left(p=.033, \eta_{p}{ }^{2}=.04\right)$, the pedophiles were less 
exclusive than the hebephiles $(p=.015, d=0.52)$. All of the effect sizes for sexual interest in males were in the medium range.

When the MANOVA was conducted for sexual interest in females (bottom panel of Table 6) the phallometric differences scores differentiated the groups $\Lambda=.97, F(6,1940)=5.27$, $p<.001, \eta_{p}{ }^{2}=.02$. The difference score between the pubescent girls and adult women $(p=.027$, $\left.\eta_{\mathrm{p}}{ }^{2}=.01\right)$ and between the prepubescent girls and boys $\left(p<.001, \eta_{p}{ }^{2}=.02\right)$ were significant. Contrasts revealed that the only significant difference was between the hebephiles and the teleiophiles. For the difference score between pubescent girls and adult women, there was a small effect where hebephiles were less exclusive than the teleiophiles $(p=.021, d=-0.17)$. For the difference score between the prepubescent girls and boys, the teleiophiles had less exclusivity than the hebephiles $(p<.001, d=0.34)$.

Table 6.

Phallometric Difference Z Scores Separated by Erotic-Gender Interest

Hebephile $\quad$ Pedophile $\quad$ Teleiophile

\section{Sexual interest in}

males

Prepubescent girls

$.65(.48)$

$.68(.59)$

$.43(.41)$

versus boys

Prepubescent versus

$.42(.38)$

$.72(.54)$

$.52(.43)$

pubescent boys 
Pubescent boys versus

$.86(.59)$

$.59(.43)$

$.92(.63)$

adult men

\section{Sexual interest in}

\section{females}

Prepubescent girls

$.71(.51)$

$.68(.55)$

$.55(.43)$

versus boys

Prepubescent versus

$.50(.39)$

$.59(.43)$

pubescent girls

Pubescent girls versus

$.71(.51)$

$.69(.51)$

$.81(.64)$

adult women

Note. Numbers in boldface indicate the lowest score for that category, which indicates less differentiation between the two sets of stimuli. The top panel refers to analyses that were restricted to sexual interest in males and the bottom panel refers to analyses that were restricted to offenders with a sexual interest in females.

\section{Discussion}

When examining a heterogeneous sample of sexual offenders including both admitters and deniers, there was evidence of convergent validity for the various measures of pedophilia and hebephilia. Despite findings of convergent validity, the various measures of hebephilia and pedophilia showed substantial overlap throughout all analyses. Further, the pattern of convergence between pedophilia and hebephilia is supported by the very few differences that emerged when comparing the pedophiles and hebephiles on sexual arousal to potentially erotic 
stimuli. This pattern is in contrast to the teleiophiles, as hebephiles had greater levels of sexual arousal to stimuli of children and less arousal to stimuli depicting fully mature adults.

The second hypothesis that hebephiles would be more likely to be non-exclusive in their sexual interest was not fully supported. When examining sexual interest in females, the only notable difference was that the teleiophiles were less exclusive than the hebephiles in sexual arousal to prepubescent boys compared to prepubescent girls. When examining sexual interest in males, the pedophiles were less exclusive than the hebephiles in their arousal to pubescent boys compared to adult men, whereas the hebephiles were less exclusive than the pedophiles in their response to pubescent versus prepubescent boys.

\section{Hebephilia as a Sexual Interest}

The present results are consistent with the wider body of literature that suggests there are men who are sexually interested in pubescent children and whose sexual arousal patterns are consistent with their self-report (e.g., Blanchard, Lykins, et al., 2009; Beier et al., 2009, 2013). The analyses provide strong evidence for convergent validity for hebephilia, as all indicators were positively and significantly correlated, with higher scores on the hebephilia index associated with self-report and sexual behaviour indicators of hebephilia. This provides additional support for the validity of hebephilia, even when including deniers, who have been excluded from previous research. Additionally, consistent with meta-analytic data (e.g., Chivers et al., 2010) the intercorrelations suggest there is a positive and significant relationship between self-reported sexual interest and sexual arousal, even when including deniers in analyses.

The AUC analyses provided further evidence that higher scores on the hebephilia index were associated with self-report and behavioural indicators of hebephilia. Overall, large effect sizes were observed for the prediction of the various indicators of pedophilia and hebephilia. The 
only exception to this was the prediction of $3+$ young teen victims. There was only a medium effect observed for the prediction of $3+$ young teen victims by the hebephilia index. This suggests that sexual offending against young teen victims might not correspond as strongly to hebephilia as other indicators of sexual interest. This is somewhat in line with previous research that has suggested that offenders with adolescent victims are more likely to have victims from other age ranges and that other factors such as opportunistic offending might partially explain offending against young teen victims (e.g., Guay, Proulx, Cusson, \& Ouimet, 2001; Heil, Ahlmeyer, \& Simons, 2003).

Finally, analyses that directly compared hebephiles to pedophiles and teleiophiles on sexual arousal to potentially erotic stimuli suggested that hebephiles significantly differed from teleiophiles. Hebephiles had greater response to depictions of children and lower levels of response to depictions of adults than teleiophiles. There were not many differences between pedophiles and hebephiles in sexual arousal to potentially erotic stimuli and when differences emerged they tended to be in the small effect range. Further, pedophilia and hebephilia loaded on to the same factor in the PCA analysis, whereas teleiophilia loaded on to another factor. This is consistent with research that has found that hebephiles are similar to pedophiles, both of whom differ from teleiophiles on a variety of correlates (e.g., intelligence; Cantor et al., 2004). Taken together, these results suggest that hebephiles are distinct from teleiophiles.

\section{Overlap between Pedophilia and Hebephilia}

A consistent finding across all of the analyses was significant overlap between pedophilia and hebephilia. Large effects were observed for the correlations between the various indicators of the two constructs. It is of note that the effect sizes were large when examining the same type of indicator for the two constructs (e.g., self-reported sexual interest in prepubescent and 
pubescent children) and medium when examining different types of indicators for the two constructs (e.g., self-reported sexual interest in pubescent children and the pedophilia index). It is likely that method covariance partially explains the large correlations between the same types of indicators; however, the correlation between pedophilia and hebephilia when examining the different types of indicators suggests that method covariance is not the sole explanation. In further support of the overlap independent of method covariance, the phallometric indices predicted all of the indicators of pedophilic and hebephilic sexual interest. Finally, very few differences emerged when contrasting the pedophiles and hebephiles on sexual arousal to various stimuli and they loaded on to the same factor in the principal component anyalsis. Thus, pedophiles and hebephiles are more similar than they are different, which supports the recommendation of Blanchard and colleagues (2009) who argued that pedophilia be expanded to include hebephilia. Their proposal included a specifier for pedophebephilia (i.e., sexual interest in both prepubescent and pubescent children), which seems particularly warranted given the overlap between pedophilia and hebephilia.

The overlap between pedophilia and hebephilia is further supported by the PCA where self-report and sexual behaviour indicators loaded onto the same factor, whereas sexual arousal indicators loaded onto the second factor. It is unclear why the self-report and sexual behaviour indicators loaded on to the same factor; however, one hypothesis is that men who admitted to sexual interest in children might have been more inclined to admit to sexually offending against child victims. Further, sexual interest in multifaceted and it may be that sexual arousal and selfreport tap into one aspect of sexual interest, whereas sexual arousal taps into another aspect of sexual interest. Another observation from the PCA that supports the overlap between the two constructs is that pedophilia and hebephilia (self-report and behaviour) loaded on to the same 
factor, whereas teleiophilia (as indicated by loadings of phallometric scores that signified greater interest in adults relative to children) loaded on to the second factor.

Interestingly, there have also been findings that suggest hebephiles are more likely than pedophiles and teleiophiles to be non-exclusive in their sexual interest (e.g., Beier et al., 2013). The idea of non-exclusivity is consistent with the DSM (APA, 2013) and is further supported by studies that have shown non-exclusivity in phallometric profiles and self-report (e.g., Barbaree \& Marshall, 1989; Beier et al., 2013; Blanchard et al., 2012). The concept of non-exclusivity is partially supported, due to the overlap between pedophilia and hebephilia. Nonetheless, this pattern of overlap does not explicitly address whether non-exclusivity is more applicable to hebephilia. Thus, explicit analyses on the difference scores were crucial in examining whether non-exclusivity was more pertinent to hebephilia.

The difference score analyses only provided partial support for the finding that nonexclusivity is greatest in hebephiles (e.g., Beier et al., 2009, 2013). Specifically, non-exclusivity among hebephiles was entirely dependent on the set of stimuli that were contrasted. The only comparison that provided support for this hypothesis was that hebephiles were less exclusive than pedophiles in their sexual arousal to pubescent and prepubescent boys, when specifically examining androphilic sexual interest. This is not surprising as Blanchard, Lykins, and colleagues (2009) reported less exclusivity in arousal patterns among those who self-reported sexual interest in pubescent boys. Additionally, there were very few differences between the hebephiles and teleiophiles on exclusivity in sexual arousal. This is consistent with findings that suggest there is a steep gradient in phallometric responding for teleiophiles who tend to have greater levels of exclusivity (Beier et al., 2009, 2013; Blanchard et al., 2012; Lykins et al., 2010a). 
Despite findings in support of non-exclusivity in hebephiles, there were also findings that were contrary to the hypothesis. In three instances, teleiophiles and pedophiles were less exclusive than hebephiiles. Interestingly it appeared that the farther the difference scores were from the preferred set of stimuli, the greater the level of non-exclusivity in sexual arousal. Specifically, the teleiophiles were less exclusive compared to the hebephiles, when contrasting the response to prepubescent girls and boys. Further, pedophiles were less exclusive than hebephiles when comparing sexual arousal to pubescent boys and adult men. This could either mean that prepubescent girls and boys would be of equal interest to teleiophiles and pubescent boys and adult men would be of equal interest to pedophiles or that phallometric profiles flatten out the farther the criterion stimuli are from preferred stimuli (Blanchard et al., 2012). Blanchard and colleagues (2012) argued that there might be a floor effect if the stimulus being viewed is significantly different from the individual's most preferred stimulus and would require higher levels of sexual arousal to detect meaningful levels of differentiation.

The findings on non-exclusivity have two implications. First, it might be more useful to consider non-exclusivity in regards to specific sets of stimuli as opposed to an overall measurement, as overall measurement would mask important differences between different classes of stimuli. Additionally, there is a need for future research on how best to capture nonexclusivity and to examine its implications for the wider field of clinical sexology and clinical forensic psychology. Further, the findings in the present study are not entirely consistent with Beier and colleagues $(2009,2013)$ who found that non-exclusivity is more common in hebephiles. Nonetheless, Beier and colleagues did not specifically examine patterns of overlap as it pertained to hebephilia, and it is possible that non-exclusivity is particular to the overlap between the pedophilia and hebephilia. 


\section{Implications for the Construct of Hebephilia}

The results of the present study provide support for Blanchard, Lykins, and colleagues (2009) argument that pedophilia be expanded to explicitly include hebephilia in the diagnostic criteria. The present results suggest hebephilia has convergent validity and significantly overlaps with pedophilia. Although the present study does not address functional impairment (i.e., the second aspect of whether a condition is a mental disorder), there is additional support for the inclusion of hebephilia alongside pedophilia, based on studies that have found self-reported hebephiles can be distressed by their sexual interest (e.g., Beier et al., 2009, 2013). Nonetheless, many might express concern over this recommendation, especially those who are concerned about pathologizing normative sexual interests. Although this study does not directly address whether hebephilia would meet the threshold of harmful dysfunction as outlined by Wakefield (1992), it does suggest pedophilia and hebephilia are more similar than they are different. As such, it would likely not provide the entire clinical picture if hebephilia were excluded from the diagnostic manual. For example, if an individual appears at a clinic with sexual interest in both prepubescent and pubescent children, assigning a label of pedophilia does not address that the individual also has significant interest in pubescent children, which would also be illegal if acted on. Even though the DSM is not concerned with whether something is legal, the fact that if acted on it would likely result in legal problems means that it could be classified objectively as impairment. Nonetheless, there is a need for additional research on the construct of hebephilia and whether it meets the criterion of a mental health disorder set out by Wakefield (1992). This research will be important for guiding further revisions of the DSM and the upcoming ICD revisions. 


\section{Limitations and Future Research Directions}

Prior to discussing future research directions, a number of limitations need to be addressed. In the present study, the sample was quite large, but power was lost when examining male sexual interest separately. For example, there were only nine individuals sexually interested in prepubescent boys. To a similar extent, the issue of sample size also applies to the categorical sexual interest variable that was used in the MANOVA analyses. Approximately half of the sample were not entered into analyses, as they denied sexual interest in children and had fewer than three child victims. Given the importance of conducting analyses on homogenous groups that would be pedophilic, hebephilic, or teleiophilic, the choice was made to exclude these ambiguous cases from the MANOVA analyses. Similarly, with the categorical variable we attempted to classify men who were pedohebephilic by considering their stronger sexual interest, which essentially eliminated the group of pedohebephiles from analyses. Nevertheless, the majority of men who were initially classified as pedohebephiles had a predominant sexual interest, which was used for classification purposes. Finally, the present study did not include depictions of older adolescents in phallometric stimuli and an examination of ephebophilia was not possible, including the impact this would have had on analyses pertaining to non-exclusivity.

Future research should examine the underlying structure of erotic-age interest. More advanced modeling approaches such as taxometric analysis may be useful in this regard (e.g., Mackaronis et al., 2011; Schmidt et al., 2013), utilizing other measures of sexual interest such as phallometry. Further, it would also be important to examine whether the exclusive and nonexclusive distinction can be adequately captured and has any clinical utility. This distinction would likely have clinical utility as men with non-exclusive sexual interest would likely have an expanded victim pool, which may translate into a greater number of victims and higher levels of 
recidivism. Finally as previously stated, there is a need for additional research or consensus in the literature on whether hebephilia is pathological and meets Wakefield's (1992) definition of a mental disorder that guides the DSM. Similarly, it is important to examine whether the expansion of pedophilia to include hebephilia would have on forensic practice in applied settings (e.g., impact on rates of men who are subject to civil commitment).

\section{Summary of Study 1}

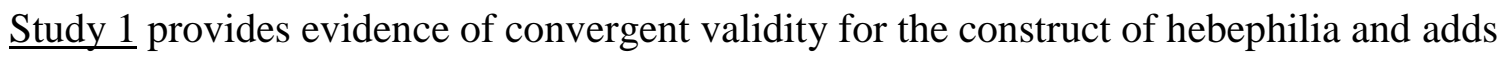
to a growing body of literature that provides support for the validity of the construct. The patterns of sexual arousal for hebephiles during phallometric testing were as expected and the hebephilia index predicted self-report and behavioural indicators of hebephilia. The present study expands on previous work by including deniers in all analyses and examining patterns of nonexclusivity in phallometric data. Interestingly, the correlations and non-exclusivity analyses suggest there is considerable overlap between pedophilia and hebephilia as evidenced by strong correlations between the two constructs. Further, hebephilia predicted indicators of pedophilic sexual interest (e.g., sexual offending against three or more child victims) and similarly pedophilia predicted indicators of hebephilic sexual interest (e.g., sexual interest in pubescent children).

With the establishment of convergent validity, both $\underline{\text { Study } 2}$ and $\underline{\text { Study } 3}$ specifically examine the application of these various methods of assessing hebephilia and their association with sexual offending. Even though there is evidence to suggest that hebephilia is a valid construct, there is a need for further research on how it might relate to sexual offending victim choice. This would be helpful in providing information on the concurrent validity of the construct if hebephilia was associated with selection of victims who were young teens (i.e., 
between the ages of 11 to 14, which would approximate to Tanner Stages 2 or 3 of sexual development). Additionally, the way the overlap between hebephilia and pedophilia translates into selection of victims who are multiple age ranges is of particular interest. 


\section{Chapter 3}

\section{Study 2: Hebephilia and Victim Age Selection}

As previously discussed in Chapter 1, hebephilia was not included in DSM-5 as a standalone diagnosis, partially due to concerns regarding the validity of the construct. There is a need for further research on hebephilia in order to examine its validity. One way to examine the validity of hebephilia is to examine its relationship with past sexual offences against children. In many countries, acting on hebephilic sexual interests is illegal because the age of consent for sexual activity is 14,16 , or higher, and most pubescent children would be between the ages of 11 and 14. For example, in Canada the age of consent for sexual activity was raised from age 14 to age 16 (see the Criminal Code of Canada for further information). Nevertheless, given findings pertaining to the exclusivity of sexual interest it is possible that hebephiles would be more likely than pedophiles or teleiophiles to select victims of different age ranges. The present study examined the relationship between hebephilia and sexual victim age choice.

\section{Hebephilia and Sexual Victim Ages}

Despite the extensive research on pedophilia and sexual offending, there has been limited research conducted on hebephilia (operationalized as a sexual interest) and sexual offending. Nevertheless, the relationship between other erotic-age interests and sexual victim age is well established. For example, it has been argued that approximately 50 to $60 \%$ of child molesters are pedophilic (Seto, 2008), and there has been significant research effort to examine how pedophilia translates into victim choice. There is a need for additional research that specifically examines the relationship between hebephilia and victim selection.

Given previous findings that hebephiles are less likely to be exclusive in their sexual interest (Beier et al., 2009) and the considerable overlap between pedophilia and hebephilia (see 
chapter 2), it is possible that hebephiles might be more likely to have victims of different age ranges, referred to as victim age polymorphism (i.e., having victims in different age categories; Guay, Proulx, Cusson, \& Ouimet, 2001). There are at least two reasons to expect that hebephiles may be more likely to be polymorphic. First, typically assessors are only aware of victim ages, not about the Tanner Stage of sexual development of sexual offense victims. Someone with hebephilia may be polymorphic because he has offended against a precociously developing nine year old who is already showing signs of pubertal onset or as a 15 year old who has not begun puberty. The second reason comes from the sexual response gradient hypothesis (Blanchard et al., 2012; Seto et al., 1999), which proposes that men should show their greatest sexual arousal to their most preferred stimulus category, but should still show some sexual arousal to their next preferred category, and so forth down the gradient. Accordingly, men interested in adult women typically show their greatest sexual response to adult women, followed by older adolescent girls, young pubescent girls, and then female prepubescent children. The slope from preferred stimulus to least preferred stimulus is generally steep (Lykins et al., 2010a). Hebephiles could show a sexual response gradient in both directions, that is, from pubescent children to prepubescent children or to older adolescents and there slope between preferred and least preferred stimulus would not be as steep. This is in contrast with pedophiles who would be expected to have a steep unidirectional gradient, from prepubescent children to pubescent children to older adolescents to adults, whereas teleiophiles would be expected to have a unidirectional gradient in the reverse direction. If the sexual response gradient hypothesis is valid, hebephiles would again be expected to be more likely to exhibit victim age polymorphism in their offence series. 


\section{Present Study}

Given the gaps in the literature on hebephilia and sexual offending, the present study was conducted in order to establish the concurrent validity of hebephilia. In order to address validity, the present study examined whether hebephilia as assessed in a clinical setting corresponded to past sexual victim age selection and victim age polymorphism. If hebephilia is associated with past sexual offending against children, this would suggest it is crucial to assess for hebephilia among offenders with child victims. Thus, the presence of hebephilia may partially explain why an offender is targeting children. Further, these results may add further credence to the construct of hebephilia and impact future iterations of the DSM and the ICD.

In the present study, hebephilia was assessed separately via self-report and phallometric assessment. Herein the term self-report refers to responses that were given during an interview pertaining to the individual's sexual interest. The term sexual arousal is used to describe the results of the phallometric assessment. Although in theory sexual interest and sexual arousal correspond (e.g., $r=.66$; Chivers et al., 2010), sexual offenders are often motivated to deny sexual interest in children resulting in low correspondence between the two constructs. As such, sexual interest and arousal are examined separately in all analyses.

In the present study it is hypothesized that sexual arousal and self-reported hebephilic interest would be associated with selecting young teen victims (age range of 11 to 14). Similarly, pedophilic interest would correspond to victim selection for children. The second hypothesis is that hebephilia would be associated with having victims of other age ranges (i.e., victim age polymorphism). 


\section{Method}

\section{Sample}

$\underline{\text { Study } 2}$ is based on the same sample of 2,238 male sexual offenders from Study 1 who were referred to the SBC (see chapter 2 for more information). Even though the sample and measures are the same for the two studies (i.e., measures were not re-administered), the study

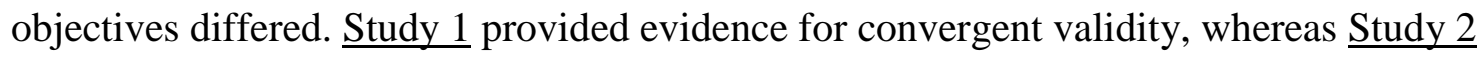
examined the relationship between hebephilia and sexual victim age selection. As such, $\underline{\text { Study } 2}$ examines concurrent validity as it applies to hebephilia.

\section{Measures}

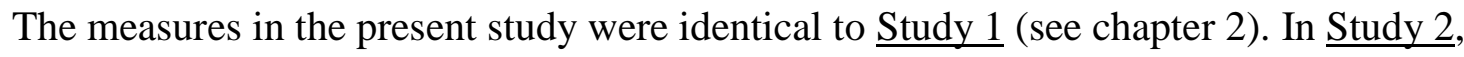
sexual arousal (phallometric assessment) and self-report (interview data) were independent variables and past sexual behaviour (admissions or criminal charges) was the dependent variable. It is of note that both the sexual arousal and self-report variables were continuous and included both those who admitted and denied sexual interest in children. Even though the same measures and data used are the same across the studies, the procedure for the creation of the variables differed slightly in $\underline{\text { Study } 2}$.

\section{Procedure}

Sexual arousal. A pedophilia and a hebephilia index were calculated based on patterns of sexual arousal. These indices were also calculated separately for both male and female stimuli and were continuous for all analyses. Despite the high correlation between the two constructs in Study 1 , the indices will not be combined as the focus of the dissertation is on examining hebephilia separately from pedophilia. Further, despite strong positive correlations between the two constructs, there may still be crucial differences between them in relation to victim choice. 
Self-report. Information gathered from interviews on self-reported erotic age interest was treated as another measure of sexual interest. The self-report sexual interest variables were also examined separately, based on erotic-gender interest. These ratings were kept continuous for all analyses and were reverse coded so that a score of 5 indicated the strongest sexual interest in that specific category.

Victim choice. Interview and file information were used to classify offenders based on their sexual offending history into the following categories: offences against children (10 and younger), offences against young teens (11 to 14), offences against older teens (15 to 16), and offences against adults (17 and older). The gender of the victim for each victim age group was also taken into account. The age cut-offs were chosen in order to provide a best approximation of the Tanner Stages (see chapter 1).

The determination of polymorphism was based on the offender having victims from two or more age categories. It is important to note that older teens and adults were included as one category. Older adolescent victims or those in Tanner Stage 4 more closely resemble adults who have reached sexual maturity. Further, sexual interest in older adolescents is not necessarily sexually atypical (Hames \& Blanchard, 2012); however, this does not mean that sexual behaviour with older adolescents is socially acceptable.

\section{Data Analysis}

Between-group significance testing was conducted for all analyses. Linear regressions were conducted separately for sexual interest and sexual arousal variables in order to assess the association of erotic-age interest and various variables pertaining to victim selection. Separate linear regression was conducted for each of the victim age categories. In analyses pertaining to polymorphism, only those with two or more victims were entered into analyses $(n=1,335$ or 
$60 \%$ of the sample). All polymorphism analyses were conducted using logistic regression as polymorphism was a dichotomous variable.

Measures of effect size were considered in the interpretation of all results, as they provide a measure of the relative magnitude of the effect. Effect sizes were important to consider due to the large sample size, which makes it easier to detect statistical significance. In the present study, a small effect size is $R^{2}=.01$, a medium effect is $R^{2}=.06$, and large effect is $R^{2}=.14$ (Cohen, 1988; Fritz et al., 2012). Further, for correlations a small effect is $r<.20$, a medium effect is .20 to .30 and a large effect is greater than .30 (Hemphill, 2003). Standardized betas are interpreted as a measure of effect size for the regression analyses (Hemphill, 2003). Medium to large effects will be the primary focus of the discussion as these effects are more substantive.

\section{Results}

\section{Descriptive Information}

The average score on the hebephilia index was $-0.10(S D=0.86)$, and the average score on the pedophilia index was $-0.46(S D=0.95)$. When separated by gender interest, the average male hebephilia index score was $0.05(S D=0.62)$, the average female hebephilia index score was $-0.10(S D=.89)$, the average male pedophilia index score was $0.15(S D=0.58)$, and the average female pedophilia index score was $-0.57(S D=0.99)$. These variables were within the acceptable range for skewness $|2|$ and kurtosis $|7|$ (Fields, 2013).

The average rating for hebephilic sexual interest was $1.58(S D=1.21)$ and the average rating for pedophilic sexual interest score was $0.78(S D=3.80)$. When examined separately by gender, the average female hebephilic sexual interest score was $1.46(S D=1.08)$, the male hebephilic sexual interest score was $1.18(S D=0.73)$, the female pedophilic sexual interest average score was $0.67(S D=4.46)$ and the male pedophilic sexual interest score was $1.09(S D=$ 
0.50). All of the self-report sexual interest variables were outside of acceptable ranges for skewness and kurtosis, with the exception of self-reported hebephilic sexual interest.

Nonetheless, this did not violate the linear regression assumptions, as self-report variables were used as predictor variables only.

Descriptive information for victim choice variables are presented in Table 7. It is important to note that a number of men were referred due to exhibitionism, voyeurism, or frotterusim and had a particularly high number of victims. There were also a number of contact sexual offenders with high victim counts. As such, all victim choice variables were collapsed into variables with a range of 0 to $10+$ victims, which attempts to minimize the undue influence those with high victim counts would have on the results. Further, given the low frequency of male older teen and adult victims, these two variables were collapsed for all analyses. All of the victim variables were outside of the acceptable ranges for skewness $|2|$ and kurtosis $|7|$ (Fields, 2013). ${ }^{4}$

Table 7.

Descriptive Information on Sexual Behaviour

Dependent Variables $\quad$ Mean $(S D) \quad$ \% who had one or more
victims

\begin{tabular}{llc}
\hline Child victims & $1.17(1.83)$ & $55 \%$ \\
Young teen victims & $0.92(1.75)$ & $46 \%$
\end{tabular}

\footnotetext{
${ }^{4}$ Based on skewness and kurtosis values and that the outcome variable was victim counts, a series of binomial regressions were also conducted in order to address any potential violations of linear regression. Results for the binomial regression were consistent with the results of the linear regression.
} 
Older teen victims

Adult victims

Boy child victims

Girl child victims

Boy young teen victims

Girl young teen victims

Boy older teen victims

Girl older teen victims

Adult men victims

Adult women victims
$0.43(1.50)$

$1.05(2.52)$

$0.33(1.07)$

$0.87(1.57)$

$0.28(1.14)$

$0.66(1.45)$

$0.09(0.72)$

$0.33(1.33)$

$0.07(0.57)$

$0.99(2.49)$
$17 \%$

$29 \%$

$18 \%$

$45 \%$

$11 \%$

$37 \%$

$4 \%$

$14 \%$

$3 \%$

$27 \%$

Note. Victim information was determined from official file information and self-report. Victim information was a count variable that could range from zero to ten.

\section{Erotic-Age Interest and Sexual Victim Choice}

A series of linear regressions were utilized to examine the role of erotic-age interest on sexual victim choice. These analyses were conducted for both sexual arousal and self-reported sexual interest in relation to hebephilia and pedophilia. Separate linear regressions were conducted for each victim choice category. Results are presented in Table 8.

Hebephilia. As hypothesized, having higher scores on the hebephilia index was associated with having a greater number of young teen victims. Additionally, the hebephilia index was positively associated with having child and older teen victims. The opposite pattern 
was found for adult victims, as a greater number of adult victims were significantly associated with a lower score on the hebephilia index (i.e., greater sexual interest in adults compared to pubescent children). The only large effect was for child victims, the effect for young teen victims was medium, and the remainder of the effect sizes were small.

Similarly, all of the analyses pertaining to self-reported hebephilia were significant and largely consistent with the findings for hebephilic sexual arousal. A greater interest in pubescent children was associated with having a greater number of young teen victims, as well as a greater number of child and older teen victims. The only difference was that self-reported hebephilia was associated with having a greater number of victims in each age category, including adult victims. Further, effect sizes were in the large range for child and young teen victims, medium for older teen victims, and small for adult victims.

Pedophilia. The results for the pedophilia index were identical to the results for the hebephilia index. As expected, a higher score on the pedophilic index was associated with having a greater number of child victims, which was a large effect. Further, higher scores on the pedophilia index were associated with having a greater number of young teen and older teen victims, but these effects were medium and small, respectively. A higher score on the pedophilic index was associated with having significantly fewer adult victims and the effect size was medium.

A nearly identical pattern emerged for self-reported pedophilia where a higher score was associated with a greater number of child victims, young teen victims, and older teen victims. The only difference was there was no significant association with the number of adult victims and that effect sizes were large for child and young teen victims and medium for older teen victims. 
Table 8 .

Erotic-Age Interest and Sexual Victim Choice

\begin{tabular}{lccc}
\hline Victim choice category & $B(S E)$ & Standard & $F$ \\
& Coefficients & & $R^{2}$
\end{tabular}

\begin{tabular}{|c|c|c|c|c|}
\hline \multicolumn{5}{|l|}{ Hebephilia index } \\
\hline Child victims & $.48(.04)$ & .22 & $117.45^{* * *}$ & .05 \\
\hline Young teen victims & $.27(.04)$ & .13 & $39.45^{* * *}$ & .02 \\
\hline Older teen victims & $.12(.04)$ & .07 & $3.15 * *$ & .004 \\
\hline Adult victims & $-.29(.06)$ & -.10 & $22.24 * * *$ & .01 \\
\hline \multicolumn{5}{|c|}{ Self-reported hebephilia } \\
\hline Child victims & $.47(.03)$ & .31 & $236.84 * * *$ & .10 \\
\hline Young teen victims & $.45(.03)$ & .31 & $231.31 * * *$ & .09 \\
\hline Older teen victims & $.28(.03)$ & .23 & $118.93 * * *$ & .05 \\
\hline Adult victims & $.12(.04)$ & .06 & $7.46^{* * *}$ & .003 \\
\hline \multicolumn{5}{|l|}{ Pedophilic Index } \\
\hline Child victims & $.53(.04)$ & .28 & $183.51 * * *$ & .08 \\
\hline Young teen victims & $.28(.04)$ & .15 & $51.70 * * *$ & .02 \\
\hline
\end{tabular}




\begin{tabular}{lcccc}
\hline Older teen victims & $.08(.03)$ & .05 & $5.06^{*}$ & .04 \\
Adult victims & $-.27(.06)$ & -.10 & $23.60^{* * *}$ & .10 \\
& & & &
\end{tabular}

Self-reported pedophilia

Child victims

$1.10(.04)$

.47

$617.32 * * *$

.22

Young teen victims

$.49(.05)$

.22

$112.12^{* * *}$

.05

Older teen victims

$.27(.04)$

.14

$44.27 * * *$

.02

Adult victims

$.05(.02)$

.07

0.50

.00

Note. Each of the victim categories represented their own separate linear regression. Results are grouped by indicator.

$* * * p<.001, * * p<.01, * p<.05$

\section{Sexual Victim Age Selection: Impact of Gender Interest}

Hebephilic sexual arousal. A higher score on the male and female hebephilia indices were associated with a greater number of boy child victims and boy young teen victims (see Table 9). Effect sizes were medium for sexual interest in pubescent boys, but small for sexual interest in pubescent girls. Although the regression was significant for older teen/adult men victims, the predictors were not significant.

For female victims, both the male and female hebephilia indices were associated with a having greater number of girl child victims and produced small and medium effects, respectively. This is in contrast to young teen victims, as only the female hebephilic index was significantly associated with having a greater number of girl young teen victims (small effect). 
For female adult victims, there was a positive and medium sized effect with the female hebephilic index.

Table 9.

Hebephilic Index by Gender and Sexual Victim Choice

\begin{tabular}{|c|c|c|c|c|}
\hline Victim choice category & B (SE) & $\begin{array}{c}\text { Standard } \\
\text { Coefficients }\end{array}$ & $F$ and $t$ & $R^{2}$ \\
\hline Boy child & & & $39.97 * * *$ & .03 \\
\hline B hebephilic index & $.25(.04)$ & .15 & $6.75^{* * * *}$ & \\
\hline G hebephilic index & $.09(.03)$ & .08 & $3.50^{* * *}$ & \\
\hline Boy Young Teen & & & $35.26 * * *$ & .03 \\
\hline B hebephilic index & $.27(.04)$ & .15 & $6.81^{* * *}$ & \\
\hline G hebephilic index & $.08(.03)$ & .07 & $3.09 * *$ & \\
\hline Older Teen/ Adult Men & & & $3.59^{*}$ & .00 \\
\hline B hebephilic index & $.05(.03)$ & .03 & 1.45 & \\
\hline G hebephilic index & $.04(.02)$ & .04 & 1.82 & \\
\hline Girl Child & & & $37.73 * * *$ & .03 \\
\hline B hebephilic index & $.17(.06)$ & .07 & $3.07 * *$ & \\
\hline G hebephilic index & $.27(.04)$ & .15 & $7.12^{* * * *}$ & \\
\hline
\end{tabular}




\begin{tabular}{lcccc}
\hline Girl Young Teen & & & $5.25^{* *}$ & .01 \\
B hebephilic index & $.00(.05)$ & .00 & -0.03 & \\
G hebephilic index & $.11(.04)$ & .04 & $3.15^{* *}$ & \\
\hline Older Teen Girl & & & .1 .11 & .001 \\
B hebephilic index & $.03(.05)$ & .02 & 0.73 & \\
G hebephilic index & $.04(.03)$ & .02 & 1.08 & \\
\hline Adult Women & & & & \\
B hebephilic index & $-.10(.09)$ & -.03 & -1.15 & \\
G hebephilic index & $-.28(.06)$ & -.10 & $-4.59^{* * *}$ & \\
\hline
\end{tabular}

Note. In the table B stands for boy and G stands for girl. Each of the columns represents a separate linear regression.

$* * * p<.001, * * p<.01, * p<.05$

Self-reported hebephilic sexual interests. When examining self-reported hebephilia all of the regressions were significant (see Table 9). Self-reported interest in pubescent boys was significantly associated with having a greater number of boy child victims, boy young teen victims, and older teen/adult men victims. All of the effect sizes for sexual interest in pubescent boys were in the large range. For all of the results, self-reported interest in pubescent girls was negatively associated with male victim selection and produced small effects.

For female victims, greater self-reported interest in pubescent girls was associated with a greater number of girl child, girl young teen, older teen girl victims, and adult women victims. 
Effect sizes were large for girl child and young teen victims, medium for girl older teen victim, and small for adult victims. For all of these analyses, there was a negative association with male hebephilic interest.

Table 10.

Self-Reported Hebephilia and Sexual Victim Choice

\begin{tabular}{|c|c|c|c|c|}
\hline Victim choice category & Unstandardized & Standard & $F$ and $t$ & $R^{2}$ \\
\hline & Coefficients & Coefficients & & \\
\hline Boy child & & & $223.64 * * *$ & .17 \\
\hline B hebephilic interest & $.60(.03)$ & .41 & $20.97 * * *$ & \\
\hline G hebephilic interest & $-.01(.02)$ & -.01 & -0.51 & \\
\hline Boy young teen & & & $312.61 * * *$ & .22 \\
\hline B hebephilic interest & $.73(.03)$ & .47 & $24.97 * * *$ & \\
\hline G hebephilic interest & $-.05(.02)$ & -.05 & $-2.55 *$ & \\
\hline Older teen/adult men & & & $66.64 * * *$ & .06 \\
\hline B hebephilic interest & $.30(.03)$ & .24 & $11.51 * * *$ & \\
\hline $\mathrm{G}$ hebephilic interest & $-.04(.02)$ & -.05 & $-2.48 *$ & \\
\hline Girl child & & & 110.03 & .09 \\
\hline B hebephilic interest & $-.09(.04)$ & -.04 & $-2.06^{*}$ & \\
\hline
\end{tabular}




\begin{tabular}{|c|c|c|c|c|}
\hline G hebephilic interest & $.45(.03)$ & .30 & $14.83 * * *$ & \\
\hline Girl young teen & & & $100.37 * * *$ & .08 \\
\hline B hebephilic interest & $-.15(.04)$ & -.07 & $-3.62 * * *$ & \\
\hline G hebephilic interest & $.39(.03)$ & .29 & $14.09 * * *$ & \\
\hline Girl older teen & & & $64.10 * * *$ & .05 \\
\hline B hebephilic interest & $-.13(.04)$ & -.07 & $-3.45 * *$ & \\
\hline G hebephilic interest & $.29(.03)$ & .23 & $11.19 * * *$ & \\
\hline Adult women & & & $21.58 * * *$ & .14 \\
\hline B hebephilic interest & $-.32(.07)$ & -.09 & $-4.38 * * *$ & \\
\hline $\mathrm{G}$ hebephilic interest & $.27(.05)$ & .12 & $5.52 * * *$ & \\
\hline
\end{tabular}

Note. Each of the columns represents a separate linear regression. In the table B stands for boy and $\mathrm{G}$ stands for girl.

$* * * p<.001, * * p<.01, * p<.05$

Pedophilic sexual arousal. Sexual arousal to prepubescent boys and girls was significantly associated with having a greater number of boy child and young teen victims and the effects were medium and small, respectively (see Table 11). For older teen/adult men victims, a higher score on the male pedophilia index was associated with fewer male older teen/adult victims and the effect size was small; however, there was an unexpected positive association for the female pedophilia index, which produced a small effect. 
A slightly different pattern emerged for female victims. A higher score on the male and female pedophilia index was associated with having a greater number of girl child victims and effect sizes were small and medium, respectively. Nonetheless, only the female pedophilic index was associated with having a greater number of girl young teen victims, which was a small effect. Finally, a higher score on the female pedophilia index was associated with having fewer adult women victims, which was a medium effect. The regression for older teen girl victims was not significant.

Table 11.

Pedophilic Sexual Arousal and Sexual Victim Choice

$\begin{array}{lcccc}\text { Victim choice category } & \text { Unstandardized } & \text { Standard } & F \text { or } t & R^{2} \\ & \text { Coefficients } & \text { Coefficients } & & \end{array}$

\begin{tabular}{|c|c|c|c|c|}
\hline Boy child & & & $66.77 * * *$ & .06 \\
\hline B pedophilia index & $.25(.03)$ & .16 & $7.25 * * *$ & \\
\hline G pedophilia index & $.15(.02)$ & .13 & $6.06 * * *$ & \\
\hline Boy young teen & & & $9.87 * * *$ & .01 \\
\hline B pedophilia index & $.15(.04)$ & .09 & $4.03 * * *$ & \\
\hline G pedophilia index & $.16(.03)$ & .14 & $6.11 * * *$ & \\
\hline Older teen/adult men & & & $14.40 * * *$ & .02 \\
\hline B pedophilia index & $-.11(.03)$ & -.08 & $-3.55 * * *$ & \\
\hline
\end{tabular}




\begin{tabular}{|c|c|c|c|c|}
\hline G pedophilia index & $.10(.02)$ & .11 & $5.04 * * *$ & \\
\hline Girl child & & & $41.14 * * *$ & .04 \\
\hline B pedophilia index & $.15(.05)$ & .07 & $3.00 * *$ & \\
\hline G pedophilia index & $.26(.04)$ & .16 & $7.26^{* * *}$ & \\
\hline Girl young teen & & & $8.90^{* * * *}$ & .01 \\
\hline B pedophilia index & $-.00(.05)$ & -.02 & -0.08 & \\
\hline G pedophilia index & $.07(.03)$ & .05 & $2.22 *$ & \\
\hline Girl older teen & & & $11.33^{* * * *}$ & .01 \\
\hline B pedophilia index & $-.03(.04)$ & -.02 & -0.68 & \\
\hline G pedophilia index & $.05(.03)$ & .04 & 1.68 & \\
\hline Adult women & & & 1.25 & .001 \\
\hline B pedophilia index & $-.14(.08)$ & -.04 & -1.70 & \\
\hline G pedophilia index & $-.20(.06)$ & -.08 & $-3.53 * * *$ & \\
\hline
\end{tabular}

Note. Each of the columns represents a separate linear regression. In the table B stands for boy and $\mathrm{G}$ stands for girl.

$* * * p<.001, * * p<.01, * p<.05$

Self-reported pedophilic sexual interest. A similar pattern emerged for pedophilic selfreported sexual interest (Table 12). For male victims, there was a significant association between self-reported sexual interest in prepubescent boys and a greater number of boy child 
victims, boy young teen victims, and older teen/adult men victims. Effect sizes were large for boy child and young teen victims and small for older teen/adult men victims.

Similarly, there was a significant association between female self-reported pedophilic sexual interest and having a greater number of girl child victims, girl young teen victims, older teen girl victims, and adult women victims. Effect sizes were large for the association with child victims and teen victims and small for the association with adult female victims. Male pedophilic sexual interest was negatively associated with all female sexual victim choice variables.

Table 12.

Self-Reported Pedophilia and Sexual Victim Choice

\begin{tabular}{lcccc}
\hline Victim choice category & Unstandardized & Standard & $F$ or $t$ & $R^{2}$ \\
& Coefficients & Coefficients & & \\
& Coents & &
\end{tabular}

\begin{tabular}{|c|c|c|c|c|}
\hline Boy child & & & $407.67 * * *$ & .27 \\
\hline G pedophilic interest & $-.02(.03)$ & -.02 & -0.78 & \\
\hline B pedophilic interest & $1.13(.04)$ & .52 & $27.56 * * *$ & \\
\hline Boy young teen & & & 120.95 & .10 \\
\hline G pedophilic interest & $-.07(.04)$ & -.04 & $-2.10 *$ & \\
\hline B pedophilic interest & $.74(.05)$ & .32 & $15.36 * * *$ & \\
\hline Older teen/adult men & & & $4.46^{* *}$ & .004 \\
\hline G pedophilic interest & $-.02(.03)$ & -.01 & -0.58 & \\
\hline
\end{tabular}




\begin{tabular}{|c|c|c|c|c|}
\hline B pedophilic interest & $.12(.04)$ & .07 & $2.97 * *$ & \\
\hline Girl child victim & & & 224.57 & .17 \\
\hline $\mathrm{G}$ pedophilic interest & $.98(.05)$ & .42 & $20.83 * * *$ & \\
\hline B pedophilic interest & $-.14(.06)$ & -.05 & $-2.23 *$ & \\
\hline Girl young teen victim & & & $56.41^{* * * *}$ & .05 \\
\hline G pedophilic interest & $.49(.05)$ & .23 & $10.62 * * *$ & \\
\hline B pedophilic interest & $-.21(.06)$ & -.07 & $-3.29 * *$ & \\
\hline Girl older teen victim & & & $38.98 * * *$ & .03 \\
\hline G pedophilic interest & $.38(.04)$ & .19 & $8.72^{* * *}$ & \\
\hline B pedophilic interest & $-.22(.06)$ & -.08 & $-3.83 * * *$ & \\
\hline Adult women & & & $7.42 * * *$ & .01 \\
\hline G pedophilic interest & $.25(.08)$ & .07 & $3.02 * *$ & \\
\hline B pedophilic interest & $-.35(.11)$ & -.07 & $-3.16^{* *}$ & \\
\hline
\end{tabular}

Note. Each of the columns represents a separate linear regression. In the table B stands for boy and $\mathrm{G}$ stands for girl.

$* * * p<.001, * * p<.01, * p<.05$

\section{Victim Age Polymorphism}

Of the total sample, $33 \%$ of offenders were polymorphic, meaning they had one or more victims in the following age categories: child, young teen, or older teen/adult. All subsequent 
analyses were restricted to those offenders who had two or more victims. When analyses were restricted in this manner, $56 \%$ of offenders were polymorphic. Logistic regression analyses were conducted in order to examine the association of pedophilia and hebephilia and victim age polymorphism (see Table 13).

For hebephilia, all of the analyses were significant. There was a significant association between polymorphism and hebephilia as measured by sexual arousal and self-report. This relationship was consistent for both sexual interest in pubescent boys and girls. Similarly, both the pedophilic index and pedophilic self-reported interest were associated with polymorphism; however, the relationship was not consistently found for sexual interest in prepubescent boys and girls. All of the effect sizes were small for all of the analyses.

Table 13.

Erotic-Age-Gender Interest and Victim Age Polymorphism

\begin{tabular}{|c|c|c|c|c|c|}
\hline Indicator & $\mathrm{B}(\mathrm{SE})$ & Wald & $\begin{array}{c}\text { Odd's Ratio } \\
{[95 \% \mathrm{CI}]}\end{array}$ & $\chi^{2}$ & $R^{2}$ \\
\hline
\end{tabular}

\begin{tabular}{|c|c|c|c|c|c|}
\hline Hebephilic Index & & & & $23.45 * * *$ & .02 \\
\hline B Hebephilic Index & $.18(.09)$ & $3.86 *$ & $1.20[1.00,1.43]$ & & \\
\hline G Hebephilic Index & $.24(.06)$ & $14.12 * * *$ & $1.27[1.12,1.44]$ & & \\
\hline Hebephilic Interest & & & & $44.58 * * *$ & .04 \\
\hline B Hebephilic Interest & $.33(.08)$ & $16.95 * * *$ & $1.38[1.19,1.62]$ & & \\
\hline G Hebephilic Interest & $.21(.05)$ & $17.64 * * *$ & $1.24[1.12,1.37]$ & & \\
\hline
\end{tabular}


Pedophilia Index

B Pedophilic Index

G Pedophilic Index

Pedophilic Interest

B Pedophilic Interest

G Pedophilic Interest

$.13(.08)$
$21.64 * * *$

.02

$.16(.06) \quad 3.56 \quad 1.18[0.99,1.39]$

$.19(.06)$

$10.16 * *$

$1.21[1.08,1.37]$

Note. Each of the columns represents a separate linear regression. In the table B stands for boy and $\mathrm{G}$ stands for girl.

$* * * \mathrm{p}<.001, * * \mathrm{p}<.01, * \mathrm{p}<.05$

\section{Discussion}

The present study is the first to explicitly examine hebephilia and its relationship to sexual victim choice and polymorphism. Given the strong and positive correlation between pedophilia and hebephilia reported in Study 1, it was not surprising that hebephilia and pedophilia had similar sexual victim choice correlates. More specifically, hebephilia and pedophilia consistently produced medium to large effects in their association with sexual offending against children under the age of 15 . In many of the analyses, there was an association between sexual interest in pubescent children and having a greater number of older teen victims, but effects were in the small to medium range. Further, pedophilia and hebephilia were associated with an increased likelihood for victim age polymorphism; however, the effect sizes were small and the relationship was not as consistently found for pedophilia. Further, when examining the 
standardized betas, a trend emerged where the hebephiles displayed less age specificity in victim age selection.

\section{Hebephilia and Sexual Offending Victim Age}

Erotic-age interest generally mapped onto sexual age victim choice pertaining to the age range that would most closely parallel the corresponding Tanner Stages. Pedophilia and hebephilia produced medium to large effects in their association with victims under the age of 15. Victims under the age of 15 would most likely be victims in Tanner Stages 1 through 3 of sexual development (see chapter 1, table 1). Effect sizes were generally in the medium to large range when examining the association between sexual interest in children and the number of victims under the age of 15 . Therefore, the present results provide evidence for concurrent validity for both hebephilia and pedophilia.

Gender interest was an important determinant in the selection of young teen victims. Sexual interest in prepubescent or pubescent boys and girls (measured by sexual arousal) was associated with a greater number of male young teen victims. This is in contrast to victim selection for female young teen victims where there was only an association with sexual arousal to prepubescent or pubescent girls. A different pattern was observed for child victims, as both pedophilia and hebephilia were associated with having a higher number of girl and boy child victims, regardless of gender interest. Further when examining the standardized betas, sexual arousal to prepubescent children produced similar beta weights for child and young teen victims, regardless of gender interest. This is in contrast to hebephilia, where the beta weights suggested greater gender specificity in victim selection.

The importance of gender interest in the relationship between erotic-age interest and sexual victim age choice is not surprising when one considers the Tanner Stages of sexual development 
(Tanner, 1978). It has been found that for young child victims, offenders are more likely to victimize both boys and girls than offenders with older victims (e.g., Levenson, Becker, \& Morin, 2008). Further, pedophiles were more likely to have boy victims or victims of both genders than non-pedophiles (Freund \& Barbaree, 1989; Freund \& Watson, 1991; Levenson et al., 2008; Seto, 2008; Seto \& Lalumiére, 2001) and to have interest in both boys and girls (e.g., Blanchard et al., 2012). These findings are consistent with the present results and suggest that pedophiles may be less gender specific in their victim selection (e.g., Blanchard et al., 2012). These findings can partially be understood by considering that children under the age of 11 would likely be in Tanner Stage 1 of sexual development, which is marked by an absence of secondary sex characteristic development. As children progress through puberty (i.e., Tanner Stages 2 or 3 around the ages of 11 to 14) secondary sex characteristics begin to emerge and there is greater differentiation between boys and girls in their appearance (Tanner, 1978). Taken together, this suggests that gender interest may play more of a role in victim selection for teenage victims and for those with hebephilia.

Interestingly, sexual interest in prepubescent boys and girls was associated with a greater number of male young teen victims, but only sexual interest in prepubescent or pubescent girls was associated with having a greater number of young teen girl victims. In considering this result it is important to keep in mind that victim age is an imperfect proxy of sexual development. (e.g., a 13 year-old victim may resemble a prototypical 16 year-old, or vice versa). This may be especially problematic for classifying victims as young teen victims, as the age at which children reach puberty has been decreased over the past century (e.g., Herman-Giddens et al., 2011). The issue of capturing pubertal development based on victim age is more complex for females who progress through puberty at a younger age than males (Herman-Giddens et al., 1997; Herman 
Giddens, Wang, \& Koch, 2001). This may potentially explain why differences emerged for male young teen and not female young teen victims; it is likely that many of the female young teen victims were further along in their development than the male young teen victims. Unfortunately, we were unable to investigate this possibility as there is no available information on the developmental status of victims, a common issue in research on victim age.

For some of the analyses there were unexpected positive associations between sexual interest in children and older teen and adult victims; however, these effects tended to be small in magnitude suggesting they were not substantive. Further, these results were not consistently observed across the various indicators of erotic-age interest. For example, the hebephilia index was negatively associated with adult victims, but there was a positive association between selfreport hebephilia and adult victims. There are a number of important considerations in interpreting these findings. First, sexual interest is not always synonymous with sexual offending victim choice (e.g., Seto, 2008). This is further complicated by classification based on victim age as opposed to sexual development. Further, there may be a subset of men who are relatively indiscriminate in their victim selection as other paraphilic sexual interests (e.g., sexual interest in coercive sex) would override victim selection based on victim characteristics. These offenders may be more likely to offend indiscriminately against various victim types, regardless of eroticage interest.

\section{Victim Age Polymorphism}

The hypothesis pertaining to victim age polymorphism was only partially supported, as both pedophilia and hebephilia were associated with victim age polymorphism, but the effects were small in magnitude. Nevertheless, it is of note that these findings were found for both sexual interest in pubescent boys and girls, but the relationship was not consistently found when 
examined separately by gender interest for pedophilia. It was unclear why the results were not consistent for pedophilia and this should be explored in future research. Nonetheless, these findings highlight the relatively robust finding that sexual interest in pubescent children is associated with polymorphism and that this relationship is more consistent for hebephilia than pedophilia. This is further supported by the observed pattern when examining the standardized betas that were produced for the linear regressions.

An interesting pattern emerged when examining the standardized beta weights. Specifically, for pedophilia, the beta weights suggested a steep and negative relationship in their association with victim age: the largest effects were often found for child victims, medium effects for young teen victims, and small effects for older teen and adult victims. That is, as the victims moved farther from the preferred target, the magnitude of the effect decreased. This is in contrast to hebephilia, where the standardized betas were much more consistent across the various victim age categories. Thus, the gradient was not as steep for hebephiles, as the beta weights were often similar for child and young teen victims, slightly less for older teens, and smaller for adult victims. Thus, the standardized betas suggested a trend that was consistent with the sexual response gradient hypothesis (Seto et al., 1999). The sexual response gradient would predict that pedophiles may move up their gradient to a young or even an older teen victim, but an adult victim would be significantly different from their most preferred victim type. This is in contrast to hebephiles who may be more likely to move up their gradient to select older adolescent or even adult victims if a preferred victim was unavailable or down their gradient to a child victim.

The present results are somewhat consistent with previous research that has found that hebephiles were less likely to be exclusive in their sexual interest in pubescent children in comparison to other erotic-age interests (Beier et al., 2009, 2013), which could translate into 
victim selection (Michaud \& Proulx, 2009). Researchers who have found that polymorphic offenders are more likely to have adolescent victims, have postulated that adolescent victims may serve as a viable option when the offender's preferred victim type is not accessible (Guay et al., 2001; Lussier, Leclerc, Healey, Proulx, 2007). The idea of the sexual surrogate is consistent with the sexual response gradient, as the argument is that these offenders will move up or down their gradient if their preferred victim type is unavailable (Heil et al., 2003).

It is important to note that erotic-age interest and the sexual response gradient do not provide a complete explanation for victim age polymorphism. This point is further illustrated by the small effects that were observed when conducting analyses specific to victim age polymorphism. This suggests that other factors would explain additional variance in victim age polymorphism. Various models have been proposed to explain victim age polymorphism in sexual offending (Guay et al., 2001). Explanations for polymorphism include findings that these offenders are more psychopathic and the idea that polymorphism may be driven by a need for sexual sensation seeking (Porter et al., 2000; Skorvan, Huss, \& Scalora, 2010).

Nonetheless, there are two possible explanations that have not been fully explored in the literature on polymorphism. First, there is wide heterogeneity in sexual development in children, particularly around pubescence. It is possible that polymorphism is a mere reflection of the fuzzy boundaries of sexual development, because victim age is an imperfect proxy of sexual development. This is further bolstered by findings that suggest that offenders with adolescent victims are more likely to be polymorphic (Guay et al., 2001; Lussier et al., 2007). Another possible explanation is that those with indiscriminate arousal patterns identified in previous phallometric studies (Barbaree \& Marshall, 1989) are more likely to be polymorphic. In support of this hypothesis, Michaud and Proulx (2009) found that an indiscriminate arousal pattern 
during phallometric testing (i.e., non-exclusive sexual interest) was associated with victim age polymorphism. Nonetheless, their phallometric stimuli conflated violence and sexual interest in children. Future research should be conducted on the factors that contribute to polymorphism, including an assessment of underlying sexual interests among polymorphic offenders. This is an important area of future research given the high risk these offenders pose to the community (e.g., Parent, Guay, \& Knight, 2011).

\section{Implications}

Clinical Sexology. The present set of results needs to be contextualized within the wider debate pertaining to the inclusion of hebephilia in the $D S M-5$. Overall, the present study suggests that both pedophilia and hebephilia, whether measured as a self-reported sexual interest or via sexual arousal patterns, have similar correlates in terms of sexual victim age. Further, the high degree of overlap between pedophilia and hebephilia may explain previous studies in which pedophilia and hebephilia were not differentiated on various neuropsychological correlates (e.g., IQ; Cantor et al. 2004, 2005) or in functional brain imaging research (e.g., Cantor et al., 2008, 2012). Thus it is argued that the present study results fit into a wider body of literature that suggests there is significant overlap among these two constructs and they may be difficult to tease apart. This provides support for the recommendation of Ray Blanchard and colleagues $(2009,2010)$ to expand the diagnosis of pedophilia to pedohebephilia with a specifier that would indicate the focus of the erotic-age interest. Further, the specifier would include pedohebephiles (i.e., those men who are interested in both prepubescent and pubescent children), which is particularly supported by the high degree of overlap between the two constructs.

Clinical Forensic Psychology. The present study has a number of implications for the assessment and treatment of sexual offenders. Most importantly it is crucial to determine if 
hebephilia is present during sexual offender assessments, especially given the relationship between hebephilia and sexual offending against those who are under the age of consent. This should include a comprehensive assessment that includes a clinical interview with questions about sexual interest in pubescent children, a review of file information, including sexual offense history, and if available specialized techniques such as phallometric assessment. Phallometric assessment should only be used in the detection of hebephilia if stimuli specific to Tanner Stage 2 or 3 are included, as hebephiles may appear non-deviant in the absence of pubescent stimuli (Harris et al., 1992). Further, as is typical in the broader field of clinical assessment, support for the presence of hebephilia would be strongest if there was convergence between measures of erotic-age interest.

Given that pedophilia is addressed in current sexual offender treatment programs, there is no reason why hebephilia could not also be addressed. Beier et al. $(2009,2013)$ reported there exist genuinely hebephilic men distressed by their sexual interest in pubescent children who are motivated to seek treatment. This was the basis of the Dunkelfeld program developed for nonoffending pedophiles and hebephilies. In their program patients learned to build victim empathy, to employ cognitive strategies aimed at problematic perceptions, and learned strategies that they could use to prevent the occurrence of child sexual abuse. In many cases sex drive reducing medication were also available and used to help patients control their sexual impulses (Amelung, Kuhle, Konrad, Pauls, \& Beier, 2012). This is one example of a program that specifically targets hebephilic sexual interest; it is currently being evaluated.

\section{Limitations}

A number of limitations should be considered when interpreting the results of the present study. First, victims were categorized based on age as opposed to developmental status. This 
may be particularly problematic when examining hebephilia as preferred victims would likely be between the ages of 11 to 14 where the boundaries of puberty are quite fuzzy (e.g., HermanGiddens et al., 1997; Herman-Giddens, Wang, \& Koch, 2001). Further, the categorization of victims by age as opposed to sexual development could have impacted the findings pertaining to polymorphism. For example, even though hebephilia was associated with having a greater number of child victims, it may be that hebephiles are selecting older child victims who have already begun to go through puberty and resemble children in Tanner Stage 2 or 3 of sexual development (i.e., consistent with their sexual interest). Nonetheless, the use of victim age as a proxy of Tanner Stages is an inherent limitation in much of the research on victim age selection in sexual offending. A possible way to address this limitation in future research would be to ask offenders about the level of sexual development of their victims or to request referrals include information about the development status of victims (e.g., did the 12-year old victim appear pubescent or post-pubescent).

Further, the present study used only one objective measure of sexual interest, thus relying heavily on phallometric assessment. Self-report was also used as an additional measure of erotic-gender interest; however, it is limited by social desirability. In a forensic context, men would likely be motivated to be dishonest about sexual interest in prepubescent and pubescent children, because it would be associated with negative consequences. Notwithstanding this limitation, the effect sizes for self-reported sexual interest in children were often larger than the sexual arousal indicators suggesting that for those men who do admit to sexual interest in children this may be a stronger predictor of victim selection. An alternative explanation for the larger effects for self-report could be that men who admit their sexual interest in children were also more likely to admit to having additional sexual offences, as the sexual victim choice 
variables also included admissions. The limitations in both of these assessment methods are partially mitigated by the inclusion of both as indicators of sexual interest in the present study and that there was often convergence among the different findings.

Finally, the study was exclusive to sexual offenders and did not include a comparison group of men without a history of sexual offenses. As such, the association between sexual offending against children and hebephilia would likely be weaker in the general population, where some hebephilic individuals may not have committed any known sexual offenses against children (and some men who have committed sexual offenses against young teens are not hebephilic). Future research should examine the prevalence of hebephilia in the general population and assess its relationship with sexual offending against children. This is particularly imperative given debates surrounding whether hebephilia is pathological in nature.

\section{Future Research Directions}

There are a number of avenues that should be explored in future research with the main focus on examining any differences between pedophilia and hebephilia. First and foremost, future research should examine whether pedophilia can be distinguished from hebephilia via different assessment methods. For example, there is increasing interest in the utility of other indirect measures of sexual interest, such as the Implicit Association Tests in determining sexual interest in children (e.g., Brown, Gray, \& Snowden, 2009). A question that remains to be addressed is whether these measures are sensitive enough to distinguish between pedophilia and hebephilia. Further in understanding the overlap, it would be useful to examine if pedophilia and hebephilia have a similar etiology as suggested in research on the neurodevelopmental hypothesis (e.g., Cantor et al., 2008) or whether there are differences in etiology. 
A second line of research should examine whether the separation of pedophilia and hebephilia has clinical utility. In the context of clinical forensic psychology, this would partially be addressed by examining whether there are differences in recidivism rates. The determination of pedophilia has clinical utility in clinical-forensic psychology as it suggests an offender is in need of further intervention to manage their higher risk to the community, as there has been substantial research that has suggested sexual interest in children is an important predictor of recidivism (e.g., Hanson \& Morton-Bourgon, 2005). It is likely that hebephilia would have clinical utility, given its relationship with sexual offending against children, which suggests it would be worthwhile to address in treatment. Nonetheless, there is a need for research that specifically examines its relationship with recidivism. A related area of inquiry would be to examine whether pedophiles and hebephiles differ in terms of treatment responsiveness. For example, if hebephiles are less exclusive in their sexual interest towards pubescent children as found in previous studies (e.g., Beier et al., 2009), it may be easier to shift their sexual interest to age appropriate peers, which may in turn reduce rates of recidivism.

\section{Summary of Study 2}

$\underline{\text { Study } 2}$ examined the concurrent validity of hebephilia by examining its relationship to sexual victim age choice and victim age polymorphism. Results suggest hebephilia and pedophilia had similar associations with victim age selection. Similar to pedophilia, hebephilia was associated with having a greater number of victims under the age of 15 . Finally, polymorphism analyses suggested that both pedophilia and hebephilia were associated with having a greater likelihood of polymorphism. Taken together, these results highlight the importance of assessing hebephilic sexual interest during sexual offender assessment. 
Despite the importance of research on concurrent validity, there is a need for specific research that examines whether hebephilia has predictive validity in the prediction of sexual recidivism. This is crucial as risk to recidivism often guides the management of sexual offenders in the community and previous research has suggested that sexual interest in children is an important predictor of recidivism (e.g., Mann, Hanson, and Thornton, 2010). 


\section{Chapter 4}

\section{Study 3: Is hebephilia related to sexual recidivism among identified adult male sex offenders?}

Paraphilias encompass anomalous sexual interests to non-consenting individuals, objects, or situations (American Psychiatric Association, 2013). In some instances, acting on these interests could constitute illegal behaviour (e.g., acting on pedophilia by accessing child pornography or having sexual contacts with children). Paraphilic sexual interests have received significant attention as meta-analyses have found that they are one of the most robust predictors of sexual recidivism in both adolescent and adult sexual offenders (e.g., Hanson \& Bussiére, 1998; Hanson \& Morton-Bourgon, 2004, 2005; Mann et al., 2010; McCann \& Lussier, 2008). The importance of paraphilic sexual interests has led Mann and colleagues (2010) to conclude that it is a psychologically meaningful causal risk factor, because it fits into contemporary psychological theories of sexual offending and is viewed as an etiological factor in sexual offending.

\section{Sexual Interest in Children and Recidivism}

Despite these findings, paraphilic sexual interest is a broad term. As such, researchers have focused on elucidating the relationship between different paraphilias and recidivism. One paraphilia that has received significant attention is pedophilia. Large meta-analyses have confirmed the important role of sexual interest in children in the prediction of sexual recidivism (Hanson \& Bussiére, 1998; Hanson \& Morton-Bourgon, 2005; Mann et al., 2010). The relationship between sexual interest in children and sexual recidivism is particularly robust when it is assessed using phallometry (e.g., Hanson \& Morton-Bourgon, 2005; Laws, 2009; Mann et al., 2010). Specifically, the relationship between phallometrically assessed sexual interest in 
children and sexual recidivism produces a small effect size $(d=0.24,95 \% C I$ 0.16- 0.47 ; Hanson \& Morton-Bourgon, 2004).

The majority of research examining the association between sexual interest in children and sexual recidivism has been focused on pedophilia. Despite these meta-analytic findings, there have been discrepancies in individual studies. Some studies have reported that pedophilia as assessed by phallometry did not predict sexual recidivism (e.g., Kingston, Firestone, Wexler, \& Bradford, 2008; Looman, Morphett, \& Abracen, 2012; Wilson, Abarcen, Looman, Picheca, \& Fergurson, 2011), whereas others have found that it predicts sexual recidivism (e.g., Firestone et al., 2000; Moulden, Firestone, Kingston, \& Bradford, 2009; Olver \& Wong, 2006; Quinsey, Rice, \& Harris, 1995). In general, studies that have found a relationship between phallometrically assessed pedophilia and sexual recidivism have tended to include assault stimuli in their depictions of prepubescent children (e.g., Moulden et al., 2009) or the sample has been comprised of relatively high-risk sexual offenders (e.g., Olver \& Wong, 2006). This signifies the importance of including a heterogeneous sample and considering potential factors that might impact this relationship.

\section{Hebephilia and Recidivism}

In consideration of potential factors that might moderate the relationship between sexual interest in children and recidivism, there have been very few studies that have distinguished between sexual arousal to prepubescent (pedophilia) or pubescent children (hebephilia). This is of note as sexual interest in children could encompass sexual interest in prepubescent children and/or sexual interest in pubescent children. Thus, it is possible that previous studies that have not found a relationship between pedophilia and sexual recidivism may be due to the relative proportions of pedophiles and hebephiles in their offender samples. For example, if pedophilia 
but not hebephilia was predictive of recidivism, analyses could be underpowered if there were a higher number of hebephiles than pedophiles in the sample. Research on whether it is pedophilia, hebephilia, or both that predicts sexual recidivism would clarify the importance of considering hebephilia in the management of sexual offenders (e.g., risk assessment).

There have been three studies to date that have looked at the predictive validity of hebephilia in relation to sexual recidivism. In a study of child pornography offenders, selfreported hebephilic interest was an important risk factor in predicting future contact sexual recidivism, but not self-reported pedophilic interest (Eke \& Seto, 2011). Interestingly, this study could suggest that hebephilic sexual interest may be a stronger predictor of sexual recidivism in non-contact offenders than pedophilia. This relationship has not been replicated, and it is important to examine it in a sample of contact sexual offenders using more objective measures than self-report.

To date there are only two studies that have been conducted that have looked at the contribution of hebephilia to sexual recidivism in contact sexual offenders using indirect measures like phallometric responding. Canales, Olver, and Wong (2009) and Proulx, Pellerin, Paradis, McKibben, Aubut, and Ouimet (1997) both found that sexual arousal to pubescent children was predictive of sexual recidivism. Nonetheless, in these studies the sample was comprised of relatively high risk samples of men in federal facilities serving a sentence of two years or more. As such, it is unclear if findings from relatively high risk samples of male sexual offenders would generalize to a more representative sample of sexual offenders. Further, there are other ways to infer hebephilic sexual interest such as self-report (i.e., admissions) and sexual offending victim information (e.g., having a greater number of pubescent victims). It remains to be examined if these other methods of examining hebephilia would predict sexual recidivism. 


\section{Present Study}

The present study examines whether hebephilia is predictive of sexual recidivism in a sample of male adult sexual offenders who vary in their risk to reoffend. Further, it considers interest in the prediction of non-contact sexual offending (e.g., Seto, 2013) by examining the predictive validity of both pedophilia and hebephilia in relation to both contact and non-contact sexual re-offending. Finally, it examines the contribution of various potential indicators of hebephilia in the prediction of sexual recidivism: sexual arousal (measured by phallometric assessment), self-reported sexual interest, and sexual behaviour (measured by victim age).

The present study is guided by the hypothesis that pedophilia and hebephilia will be significant predictors of sexual recidivism. Further, it is expected that both pedophilia and hebephilia will be predictive of sexual recidivism taking time in secure custody into account and controlling for baseline risk level. The inclusion of analyses that look at the predictive utility of pedophilia and hebephilia above and beyond baseline risk level are important, as they provide a way to assess whether the inclusion of additional factors in addition to existing risk assessment metrics, might improve predictive accuracy.

\section{Method}

\section{Sample}

The sample was comprised of men who presented to the SBC at CAMH. The sample was initially comprised of 3,343 men who had presented to the clinic (see chapter 2 for descriptive information). Criminal record information was ordered for 1,117 offenders who met the following inclusion criteria: complete assessment information, valid phallometric profiles, assessed between 1995 and 2006 (in order to allow for adequate follow-up time), at least one 
victim of any age, offender was over the age of 18 , and the offender provided consent to the use of his data in research studies. The criminal records were received on September 27, 2013.

Of the 1,117 offenders, 273 offenders did not have a criminal record according to the organization that maintains the national database. These cases were further analyzed in order to understand why these records were not returned. It was determined that in $65 \%$ of the cases the records should have been returned as they had a charge or conviction at the time of the assessment. The remaining 35\% of cases should not have been returned for various reasons (e.g., $2 \%$ committed their index offence as a young offender and the offence would not be on their record unless they continued to offend as an adult).

In total, 844 criminal records were coded and included in the existing database. To be included in the analyses for the present study, offenders had to have one child victim 14 and under and to have one charge or conviction for a sexual offence. The final sample included 656 male sexual offenders. The average age at assessment for the sample was $41.06(S D=13.01)$. The majority ( $46 \%$ ) were referred by probation or parole, $20 \%$ were referred by their lawyers, $31 \%$ were referred by an institution (e.g., prison), and $2 \%$ were self-referred, but still had a charge/conviction as per inclusion criteria. In total, $76 \%$ had been in a significant intimate relationship (i.e., common-law or marriage) and $91 \%$ had no known psychiatric history (excluding paraphilia diagnoses). In terms of their ethnicity, $82 \%$ were Caucasian, $5 \%$ were Black, 3\% were East Indian or Pakistani, 3\% were Aboriginal, and 3\% were Asian, with 5\% indicated they were of another ethnicity. The average score on the Static-99R (i.e., risk assessment measure used to categorize offenders based on probability to reoffend that is further described below) was $1.75(S D=2.41)$ and the total follow-up time was 120.56 months $(S D=$ 27.56). 


\section{Measures}

Recidivism. Recidivism data were collected through the Canadian Police Information Centre (CPIC) maintained by the Royal Canadian Mounted Police. CPIC includes information on criminal charges and convictions that occur anywhere in Canada. It also includes information on whether the file has been referred to an immigration court or if the individual died.

Recidivism was gathered by counting criminal code convictions and charges in order to allow for the creation of composite variables including both charges and conviction.

Composite variables were created to include three main categories of recidivism for the present study. Sexual recidivism included any new sexual charge that occurred after the assessment date. Further, sexual recidivism was separated into non-contact sexual recidivism (e.g., child pornography) and contact sexual recidivism (e.g., sexual interference). Violent recidivism was coded if the offender committed a violent crime (e.g., robbery, assault). Two violent recidivism variables were included in the analysis. The first violent recidivism variable only included non-sexual violent offences. Additionally, a violent recidivism variable was created that combined non-sexual violent offences and sexual contact offences. The decision to include a violent recidivism variable combining both non-sexually violent and sexual contact offences is due to research that suggests that some non-sexually violent offences can be sexually motivated. Often, violent offences are not clearly indicated as sexual offences because of plea bargaining or prosecutorial discretion, such as when an attempted sexual assault is charged as an assault (Rice, Harris, Lang, \& Cormier, 2006).

Sexual Interest. As previously discussed (see chapter 2), volumetric phallometry was used to examine sexual arousal. Both a pedophilia index and a hebephilia index were computed. In addition to patterns of sexual arousal, self-reported sexual interests and victim information 
were also examined as a proxy of hebephilia. In the case of self-reported sexual interests, all offenders were asked about their sexual interest in prepubescent and pubescent children. Further, victim information was also utilized as previous studies have found the greater the number of child victims the more likely one is to have a sexual interest in children (e.g., Blanchard et al., 2001). All of these variables were examined separately as individual indicators of pedophilic and hebephilic sexual interest. The indicators of sexual interest were also analyzed separately by gender interest. It is of note that all of the indicators were continuous and included both those who admitted and denied sexual interest in children.

Baseline-risk level. In the field of sexual offender risk assessment, there are a wide range of tools that predict sexual recidivism. One of the most commonly used risk assessment tools, with a considerable amount of research support, is the Static-99 (e.g., Archer, BuffingtonVollum., Sterdny, \& Handle, 2006; Hanson \& Morton-Bourgon, 2009; Hanson \& Thornton, 2000; Helmus, Hanson, Thornton, Babchishin, \& Harris, 2012; McGrath et al., 2010). The Static-99 consists of 10 items: age of the offender; the presence of an intimate relationship; index or past violent; past sexual offence; prior sentencing dates; the presence of non-contact sexual offences; male victim; unrelated victim; and stranger victim. In the present study, the Static-99R was used, with the only difference from the Static-99 being that adjusted age weights are used for the first item (Helmus, Thornton, Hanson, \& Babchishin, 2012).

In the present study, the Static-99R was scored on all sexual offenders who had a sexual offence conviction or charge. The Static-99R was scored based on CPIC data and clinical files and was consistent with the coding manual.

Opportunity to reoffend. A composite score for time in secure custody was calculated by subtracting the total number of months spent in secure custody since the date of the CAMH 
assessment until the end of the follow-up period for each category of recidivism. The date for the end of the follow-up period was determined for each category of recidivism as being the date of first reoffence if the offender reoffended, or if there was no recidivism the date of death or the date the records were received. Time in secure custody included any time that the offender spent in a secure custodial setting (e.g., time in forensic hospital, time in remand, time in prison). Time in secure custody was subtracted from the total follow-up time, in order to calculate each offender's opportunity to reoffend for each type of offence, herein referred to as street time. As such, this variable not only accounted for the exact follow-up time, but the actual opportunity in months the offender had to reoffend.

\section{Procedure}

A comprehensive manual on how to code information from clinical files and criminal records was developed in order to guide the data collection process (Appendix B). A recent graduate of an undergraduate psychology program with forensic research experience was trained to assist with data entry. The research training process was extensive with the research assistant reading the Static-99R manual and scoring practice cases, familiarizing themselves with the coding manual and practicing data entry with the principal investigator. After it was determined by the primary investigator that the RA was coding as dictated by the coding manual, meetings were set up on as needed basis in order to resolve any coding issues. The RA and the author discussed any coding discrepancies or difficulties that they encountered and resolved them by consensus. If a consensus could not be reached, a supervisor (M. Seto) was consulted in order to resolve the issue, given his extensive clinical and forensic experience.

In order to establish that the data was coded consistently, inter-rater reliability was independently calculated for $10 \%$ of the cases (i.e., 90 cases). These were calculated using intra- 
class correlations (ICC) using a mixed effects absolute agreement model. As can be seen in Table 14, all of the ICC values were above 0.90 with the exception of the violent recidivism variables. For violent charges and convictions, the ICC correlations were .70 and .69, respectively.

Table 14.

Intraclass Correlations

\begin{tabular}{ll}
\hline Variable & ICC \\
\hline Assessment date & 0.99 \\
Date of index offence & 1.00 \\
Sanction associated with index offence & 0.93 \\
Total sanction time & 0.98 \\
S99R total score & 0.96 \\
Sexual contact convictions recidivism & 0.93 \\
Sexual contact charges recidivism & 0.95 \\
Sexual non-contact convictions & 1.00 \\
Sexual non-contact charges & 0.98 \\
Time in secure custody until first sexual conviction & 0.95 \\
Time in secure custody until first sexual charge & 0.95 \\
Violent conviction recidivism & 0.75 \\
Time in secure custody until first violent conviction & 0.98 \\
Violent charge recidivism & 0.92 \\
Time in secure custody until first violent charge & 0.99
\end{tabular}

Note. ICCs were calculated using a mixed effects absolute agreement model. 


\section{Data Analysis}

The predictor variables (phallometric indices, self-report, and victim information) were kept continuous for all analyses. The outcome variables were the different types of recidivism, coded dichotomously (yes/no). A series of ROC analyses were conducted in order to establish the classification accuracy of pedophilia and hebephilia in predicting various types of recidivism. Further, a series of Cox regressions with the different types of recidivism were then conducted. Cox regressions are appropriate as opportunity to reoffend (i.e., street time) is controlled, an important factor to consider when examining recidivism. Further, Static-99R scores were controlled for separately in analyses, in order to establish the effect that phallometric responding has above and beyond other factors that have been identified as important predictors of recidivism in the literature.

Measures of effect size were considered in the interpretation of all results. The present study relied heavily on AUCs and Cox regression analyses. For AUCS, a small effect is .56, a medium effect is .64, and a large effect is .71 (Rice \& Harris, 2005). Cox regressions produce odds ratios, which can be interpreted as an effect size. For odd's ratios a small effect is 1.5 , a medium effect is 3.5 and a large effect is 9.0 (Cohen, 1988). Medium to large effects will be the primary focus of the discussion.

Power analysis. Prior to data collection, a power analysis was conducted using $\mathrm{G}^{*}$ Power. Hanson and Morton-Bourgon (2005) reported a sexual recidivism rate of $14 \%$ after an average follow-up of five to six years, and produced a Cohen's D score of 0.24 between phallometrically assessed sexual deviancy and recidivism. At the time of data collection, it was estimated that the average follow-up time for the present study would be ten years. Given that $20 \%$ of offenders would be expected to sexually reoffend at ten year follow-up (Hanson, Morton, 
\& Harris, 2003), it was estimated that 205 offenders in the sample would reoffend. Based on the effect size reported by Hanson and Morton-Bourgon (2005), an estimated 196 offenders would need to reoffend in order to be adequately powered at .80 . Therefore, with a base rate of $20 \%$ the proposed study was determined to be adequately powered.

\section{Results}

\section{Descriptive Information}

In the sample, $10 \%$ reoffended sexually with a contact sexual offence and $4 \%$ reoffended sexually with a non-contact offence. There was a total sexual recidivism rate of $12 \%$ over a 10year follow-up. The non-sexual violent recidivism rate was $12 \%$. When sexual contact recidivism was included with non-sexually violent offending, the total violent recidivism rate was $20 \%$.The total non-violent recidivism rate was $29 \%$. There was an overall recidivism rate of $34 \%$ during the follow-up period.

Descriptive information for the different indicators of hebephilic and pedophilic sexual interest are presented in Table 15. For all of the sexual arousal variables, skewness and kurtosis values were within the acceptable range of $|2|$ and $|7|$, respectively (Fields, 2013). All of the sexual interest variables, with the exception of self-reported hebephilea and self-reported sexual interest in pubescent girls, were outside of the acceptable range for skewness and kurtosis. Further, all sexual behaviour indicators of pedophilia and hebephilia were outside of the acceptable range for skewness and kurtosis. Nonetheless, because the various indicators of pedophilia and hebephilia were entered as predictor variables, there were no violations of assumptions for either ROC analyses or Cox regressions. 
Table 15.

Descriptive Information

\begin{tabular}{|c|c|}
\hline Variable & Mean (SD) \\
\hline \multicolumn{2}{|l|}{ Sexual arousal } \\
\hline Hebephilia index & $-0.02(0.80)$ \\
\hline Pedophilia index & $-0.36(0.91)$ \\
\hline B hebephilia index & $0.05(0.62)$ \\
\hline G hebephilia index & $-0.01(0.84)$ \\
\hline B pedophilia index & $0.15(0.69)$ \\
\hline G pedophilia index & $-0.46(0.96)$ \\
\hline \multicolumn{2}{|l|}{ Self-Report } \\
\hline Hebephilia & $1.66(1.24)$ \\
\hline Pedophilia & $1.24(0.78)$ \\
\hline B hebephilia & $1.20(0.77)$ \\
\hline G hebephilia & $1.52(1.11)$ \\
\hline B pedophilia & $1.10(0.51)$ \\
\hline G pedophilia & $1.18(0.67)$ \\
\hline \multicolumn{2}{|l|}{ Sexual behaviour } \\
\hline Young teen victims & $1.12(1.76)$ \\
\hline Child victims & $1.38(1.81)$ \\
\hline B young teen victims & $0.32(1.11)$ \\
\hline $\mathrm{G}$ young teen victims & $0.81(1.48)$ \\
\hline B child victims & $0.40(1.15)$ \\
\hline
\end{tabular}


Note. B represents boy and $\mathrm{G}$ represents girl. For phallometric indices a positive score indicates greater response to deviant stimuli compared to non-deviant stimuli, whereas a negative score indicates a greater response to non-deviant stimuli compared to deviant stimuli. A score of 0 on these indices would represent equivalent levels of arousal to deviant and non-deviant stimuli. Self-reported sexual interest is on a 5-point scale and victim variables represent victim counts that range from 0 to 10 .

\section{Predictive Validity of Erotic Age Interests}

Sexual arousal. Contrary to hypotheses, all of the AUC values fell short of statistical significance and hovered around chance levels of prediction when examining the pedophilia and hebephilia indices (see Table 16). The highest AUCs were produced for sexual non-contact recidivism, with the pedophilia index producing an $A U C=.59, p=.125$ and the hebephilia index producing an $A U C=.61, p=.068$. Both of the AUC values were in the small effect range. Results were similar when examining the AUCs for the pedophilia and hebephilia indices separated by gender (see Table 15); however, it was found that a higher score on the female pedophilia index predicted significantly less violent recidivism. 
Table 16.

AUCs [95\% CI] for Sexual Arousal and Recidivism

\begin{tabular}{|c|c|c|c|c|c|c|}
\hline & $\begin{array}{l}\text { Hebephilia } \\
\text { Index }\end{array}$ & $\begin{array}{l}\text { Pedophilia } \\
\text { Index }\end{array}$ & $\begin{array}{c}\text { B } \\
\text { Hebephilia } \\
\text { Index }\end{array}$ & $\begin{array}{c}\mathrm{G} \\
\text { Hebephilia } \\
\text { Index }\end{array}$ & $\begin{array}{c}\text { B } \\
\text { Pedophilia } \\
\text { Index }\end{array}$ & $\begin{array}{c}\mathrm{G} \\
\text { Pedophilia } \\
\text { Index }\end{array}$ \\
\hline $\begin{array}{l}\text { Sexual } \\
\text { contact }\end{array}$ & $\begin{array}{c}.48 \\
{[.41, .56]}\end{array}$ & $\begin{array}{c}.51 \\
{[.44, .59]}\end{array}$ & $\begin{array}{c}.48 \\
{[.45, .60]}\end{array}$ & $\begin{array}{c}.48 \\
{[.41, .56]}\end{array}$ & $\begin{array}{c}.53 \\
{[.45, .60]}\end{array}$ & $\begin{array}{c}.48 \\
{[.41, .56]}\end{array}$ \\
\hline $\begin{array}{l}\text { Sexual non- } \\
\text { contact }\end{array}$ & $\begin{array}{c}.61 \\
{[.50, .72]}\end{array}$ & $\begin{array}{c}.59 \\
{[.47, .71]}\end{array}$ & $\begin{array}{c}.47 \\
{[.35, .59]}\end{array}$ & $\begin{array}{c}.61 \\
{[.50, .73]}\end{array}$ & $\begin{array}{c}.51 \\
{[.39, .62]}\end{array}$ & $\begin{array}{c}.61 \\
{[.48, .73]}\end{array}$ \\
\hline Total sexual & $\begin{array}{c}.51 \\
{[.44, .58]}\end{array}$ & $\begin{array}{c}.54 \\
{[.47, .61]}\end{array}$ & $\begin{array}{c}.47 \\
{[.40, .54]}\end{array}$ & $\begin{array}{c}.51 \\
{[.44, .58]}\end{array}$ & $\begin{array}{c}.52 \\
{[.45, .59]}\end{array}$ & $\begin{array}{c}.52 \\
{[.45, .59]}\end{array}$ \\
\hline $\begin{array}{l}\text { Total } \\
\text { violent }\end{array}$ & $\begin{array}{c}.46 \\
{[.38, .53]}\end{array}$ & $\begin{array}{c}.43 \\
{[.36, .51]}\end{array}$ & $\begin{array}{c}.53 \\
{[.47, .60]}\end{array}$ & $\begin{array}{c}.44 \\
{[.36, .51]}\end{array}$ & $\begin{array}{c}.55 \\
{[.49, .61]}\end{array}$ & $\begin{array}{c}.43^{*} \\
{[.36, .51]}\end{array}$ \\
\hline $\begin{array}{l}\text { Violent + } \\
\text { sexual } \\
\text { contact }\end{array}$ & $\begin{array}{c}.47 \\
{[.41, .53]}\end{array}$ & $\begin{array}{c}.47 \\
{[.41, .53]}\end{array}$ & $\begin{array}{c}.50 \\
{[.45, .56]}\end{array}$ & $\begin{array}{c}.47 \\
{[.41, .53]}\end{array}$ & $\begin{array}{c}.53 \\
{[.48, .59]}\end{array}$ & $\begin{array}{c}.46 \\
{[.40, .52]}\end{array}$ \\
\hline
\end{tabular}

Note. $\mathrm{B}=$ boy and $\mathrm{G}=$ girl. All AUC values fell short of significant with the exception of one

AUC

$* p<.05$

Self-report. For self-reported sexual interest, a greater interest in pubescent children was predictive of sexual non-contact recidivism and produced a medium effect size (see Table 17). When examining the results further by separating sexual interest by gender, only self-reported sexual interest in female pubescent children was associated with higher levels of sexual noncontact recidivism, but the effect size was small. This differed from pedophilic self-reported 
sexual interest, which did not contribute to the prediction of recidivism, even when analyzed separately by gender interest.

Table 17.

AUCs [95\%CI] for Self-Reported Sexual Interest

\begin{tabular}{|c|c|c|c|c|c|c|}
\hline & $\begin{array}{l}\text { Hebephilic } \\
\text { Interest }\end{array}$ & $\begin{array}{l}\text { Pedophilic } \\
\text { Interest }\end{array}$ & $\begin{array}{c}\text { B } \\
\text { Hebephilia }\end{array}$ & $\begin{array}{c}\mathrm{G} \\
\text { Hebephilia }\end{array}$ & $\begin{array}{c}\text { B } \\
\text { Pedophilia }\end{array}$ & $\begin{array}{c}\mathrm{G} \\
\text { Pedophilia }\end{array}$ \\
\hline $\begin{array}{l}\text { Sexual } \\
\text { contact }\end{array}$ & $\begin{array}{c}.50 \\
{[.43, .57]}\end{array}$ & $\begin{array}{c}.51 \\
{[.44, .59]}\end{array}$ & $\begin{array}{c}.49 \\
{[.42, .56]}\end{array}$ & $\begin{array}{c}.50 \\
{[.43, .58]}\end{array}$ & $\begin{array}{c}.51[ \\
.43, .58]\end{array}$ & $\begin{array}{c}.50 \\
{[.42, .57]}\end{array}$ \\
\hline $\begin{array}{c}\text { Sexual } \\
\text { non-contact }\end{array}$ & $\begin{array}{c}.67^{* * *} \\
{[.55, .78]}\end{array}$ & $\begin{array}{c}.58 \\
{[.45, .70]}\end{array}$ & $\begin{array}{c}.57 \\
{[.44, .70]}\end{array}$ & $\begin{array}{c}.62 * \\
{[.50, .74]}\end{array}$ & $\begin{array}{c}.56 \\
{[.44, .69]}\end{array}$ & $\begin{array}{c}.54 \\
{[.42, .66]}\end{array}$ \\
\hline $\begin{array}{c}\text { Total } \\
\text { sexual }\end{array}$ & $\begin{array}{c}.54 \\
{[.47, .61]}\end{array}$ & $\begin{array}{c}.54 \\
{[.47, .61]}\end{array}$ & $\begin{array}{c}.52 \\
{[.45, .59]}\end{array}$ & $\begin{array}{c}53 \\
{[.46, .60]}\end{array}$ & $\begin{array}{c}.52 \\
{[.45, .59]}\end{array}$ & $\begin{array}{c}.52 \\
{[.45, .59]}\end{array}$ \\
\hline $\begin{array}{c}\text { Total } \\
\text { violent }\end{array}$ & $\begin{array}{c}.45 \\
{[.38, .51]}\end{array}$ & $\begin{array}{c}.48 \\
{[.41, .55]}\end{array}$ & $\begin{array}{c}.48 \\
{[.41, .54]}\end{array}$ & $\begin{array}{c}.44 \\
{[.38, .51]}\end{array}$ & $\begin{array}{c}.51 \\
{[.44, .58]}\end{array}$ & $\begin{array}{c}.48 \\
{[.41, .54]}\end{array}$ \\
\hline $\begin{array}{c}\text { Violent + } \\
\text { sexual } \\
\text { contact }\end{array}$ & $\begin{array}{c}.48 \\
{[.42, .53]}\end{array}$ & $\begin{array}{c}.50 \\
{[.44, .56]}\end{array}$ & $\begin{array}{c}.49 \\
{[.44, .55]}\end{array}$ & $\begin{array}{c}.48 \\
{[.43, .54]}\end{array}$ & $\begin{array}{c}.51 \\
{[.45, .56]}\end{array}$ & $\begin{array}{c}.49 \\
{[.43, .55]}\end{array}$ \\
\hline
\end{tabular}

Note. $\mathrm{B}=$ boy and $\mathrm{G}=$ girl.

$* \mathrm{p}<.05 * * \mathrm{p}<.01$

Sexual behaviour. When examining sexual victim selection (see Table 18) a similar pattern to sexual arousal was observed. Both child and young teen victims were not associated with recidivism in any of the analyses. When examining victim selection by gender, none of the AUCs were significant. 
Table 18.

AUCs [95\%CI] for Sexual Behaviour

\begin{tabular}{|c|c|c|c|c|c|c|}
\hline & $\begin{array}{l}\text { Young } \\
\text { Teen } \\
\text { Victims }\end{array}$ & $\begin{array}{c}\text { Child } \\
\text { Victims }\end{array}$ & $\begin{array}{l}\text { G Young } \\
\text { Teen } \\
\text { Victims }\end{array}$ & $\begin{array}{l}\text { B Young } \\
\text { Teen } \\
\text { Victims }\end{array}$ & $\begin{array}{l}\text { G Child } \\
\text { Victims }\end{array}$ & $\begin{array}{l}\text { B Child } \\
\text { Victims }\end{array}$ \\
\hline \multirow[t]{2}{*}{ Sexual contact } & .55 & .54 & .54 & .50 & .54 & .50 \\
\hline & {$[.48, .63]$} & {$[.47, .62]$} & {$[.47, .62]$} & {$[.43, .58]$} & {$[.47, .61]$} & {$[.43, .58]$} \\
\hline \multirow{2}{*}{$\begin{array}{l}\text { Sexual non- } \\
\text { contact }\end{array}$} & .61 & .48 & .56 & .53 & .43 & .50 \\
\hline & {$[.49, .74]$} & {$[.36, .59]$} & {$[.44, .69]$} & {$[.41, .65]$} & {$[.32, .55]$} & {$[.39, .62]$} \\
\hline \multirow[t]{2}{*}{ Total sexual } & .55 & .54 & .56 & .53 & .43 & .50 \\
\hline & {$[.48, .62]$} & {$[.47, .61]$} & {$[.44, .69]$} & {$[.41, .65]$} & {$[.32, .55]$} & {$[39, .62]$} \\
\hline \multirow[t]{2}{*}{ Total violent } & $.43^{*}$ & .53 & .44 & .48 & .56 & .49 \\
\hline & {$[.46, .49]$} & {$[.46, .59]$} & {$[.37, .50]$} & {$[.41, .55]$} & {$[.49, .62]$} & {$[.42, .56]$} \\
\hline \multirow{2}{*}{$\begin{array}{l}\text { Violent }+ \\
\text { sexual contact }\end{array}$} & .48 & .54 & .48 & .50 & .56 & .49 \\
\hline & {$[.43, .54]$} & {$[.49, .60]$} & {$[.42, .54]$} & {$[.44, .55]$} & {$[.50, .61]$} & {$[.43, .55]$} \\
\hline
\end{tabular}

Note. $\mathrm{B}=$ boy and $\mathrm{G}=$ girl. None of the $\mathrm{AUC}$ values were significant.

Predictive Validity when Taking into Account Street Time

Hebephilia. For the hebephilia index (see Table 19), all beta weights were positive in their association with sexual recidivism; however, the only significant association was with sexual non-contact recidivism. For both violent recidivism variables the beta weights were negative and non-significant. A similar pattern was also found for self-reported hebephilic sexual interest with the only significant association for sexual non-contact recidivism; however, there was a negative and significant association with violent (including sexual contact) recidivism. 
Finally, for young teen victims the only significant association was with sexual non-contact recidivism. For all of the analyses the effect sizes were small.

Table 19.

Cox Regressions for Hebephilia

\begin{tabular}{|c|c|c|c|}
\hline Indicator & $\chi^{2}$ & $\mathrm{~B}(\mathrm{SE})$ & $\begin{array}{l}\text { Hazard Ratio } \\
{[95 \% \mathrm{CI}]}\end{array}$ \\
\hline
\end{tabular}

\section{Hebephilia Index}

$\begin{array}{lccc}\text { Sexual Contact } & 0.02 & .02(.16) & 1.02[.75,1.39] \\ \text { Sexual Non-Contact } & 5.54^{*} & .64(.27) & 1.89[1.12,3.20] \\ \text { Sexual Total } & 0.92 & .14(.14) & 1.15[.87,1.52] \\ \text { Violent Total } & 2.79 & -.24(.14) & 0.79[.60,1.04] \\ \text { Violent + Contact Sexual } & 0.71 & -.10(.11) & 0.91[.73,1.13]\end{array}$

\section{Hebephilia Self-Reported}

Interest

\begin{tabular}{lccc} 
Sexual Contact & 0.04 & $.02(.09)$ & $1.02[0.84,1.23]$ \\
Sexual Non-Contact & $12.92^{* * *}$ & $.41(.12)$ & $1.50[1.19,1.91]$ \\
Sexual Total & 2.81 & $.13(.08)$ & $1.14[0.98,1.34]$ \\
Violent Total & $5.20^{*}$ & $-.27(.12)$ & $0.76[0.60,0.97]$ \\
Violent + Contact Sexual & 1.29 & $-.09(.08)$ & $0.92[0.79,1.07]$ \\
\hline Young Teen Victims & & & \\
& & & $1.09[0.98,1.21]$ \\
Sexual Contact & 2.89 & $.09(.05)$ & $1.23[1.08,1.39]$ \\
Sexual Non-Contact & $10.91^{* * *}$ & $.20(.07)$ & $1.09[0.98,1.20]$ \\
Sexual Total & 2.65 & $.08(.05)$ & $0.84[0.69,1.01]$ \\
Violent Total & 3.52 & $-.18(.10)$ &
\end{tabular}


Violent + Contact Sexual $\quad 0.11 \quad-.02(.05) \quad 0.98[0.89,1.09]$

Note. Results are grouped by indicator and each column represents a separate Cox regression.

$* * * p<.001, * * p<.01, * p<.05$

Pedophilia. For the pedophilia index (see Table 20), beta weights were only positive for sexual recidivism. Nonetheless, the only sexual recidivism variable significantly associated with pedophilia was sexual non-contact recidivism which was a medium effect. An identical pattern was found for self-reported pedophilic interest, which was only predictive of sexual non-contact recidivism, but the effect size was small. The pattern of results differed for the total number of child victims, as this variable was not predictive of any type of recidivism.

Table 20.

Cox regressions for Pedophilia Index

\begin{tabular}{llcl}
\hline Indicator & $\chi^{2}$ & B (SE B) & Hazard Ratio [95\%CI] \\
\hline Pedophilia Index & & & \\
Sexual Contact & 0.98 & $.14(.14)$ & $1.14[.88,1.50]$ \\
Sexual Non-Contact & $4.74^{*}$ & $.49(.22)$ & $4.73[1.05,2.53]$ \\
Sexual Total & 3.65 & $.24(.13)$ & $1.27[0.99,1.63]$ \\
Violent Total & 3.77 & $-.25(.13)$ & $0.78[0.61,1.00]$ \\
Violent + Contact Sexual & 0.21 & $-.05(.10)$ & $0.96[0.78,1.16]$ \\
\hline Self-reported Pedophilic & & & \\
Interest & & $.08(.15)$ & $1.08[0.81,1.44]$ \\
Sexual Contact & 0.43 & $.36(.17)$ & $1.43[1.03,1.98]$ \\
Sexual Non-Contact & $5.09 *$ & $.20(.11)$ & $1.22[0.98,1.53]$ \\
Sexual Total & 3.15 & $-.17(.18)$ & $0.85[0.59,1.21]$ \\
Violent Total & 0.84 & & \\
\end{tabular}




\section{Child Victims}

Sexual Contact

2.60

$.10(.06)$

$1.10[0.98,1.24]$

Sexual Non-Contact

0.00

$.01(.12)$

$1.01[0.80,1.27]$

Sexual Total

2.13

$.08(.06)$

$1.09[0.97,1.21]$

Violent Total

0.01

$.01(.07)$

$1.01[0.88,1.14]$

Violent + Contact Sexual

1.11

$.05(.05)$

$1.05[0.96,1.15]$

Note. Results are grouped by indicator and each column represents a separate Cox regression. $* * * p<.001, * * p<.01, * p<.05$

Results by gender for hebephilia: Results were further analyzed separately by gender (see Table 21). For the male hebephilia index none of the results were significant. For the female hebephilia index the only significant result was for sexual non-contact recidivism. Violent recidivism was negatively associated with higher scores on the female hebephilia index. Results were not significant for any of the remaining recidivism categories. For male hebephilic self-reported sexual interest, there was a significant positive association with sexual non-contact recidivism. For female hebephilic self-reported sexual interest there was a significant positive association with sexual non-contact recidivism and a significant negative association with violent recidivism. Finally, for sexual behaviour none of the recidivism variables were associated with a greater number of young teen boy victims. This differed for young teen girl victims, as there was significant association with sexual non-contact recidivism. All of the significant effects produced effect sizes in the small range. 
Table 21.

Cox Regressions for Hebephilia by Gender

\begin{tabular}{|c|c|c|c|}
\hline Indicator & $\chi^{2}$ & $\mathrm{~B}(\mathrm{SE})$ & Hazard Ratio $[95 \% \mathrm{CI}]$ \\
\hline \multicolumn{4}{|l|}{ Male Hebephilia Index } \\
\hline Sexual Contact & 1.67 & $-.02(.20)$ & $0.98[0.66,1.44]$ \\
\hline Sexual Non-Contact & 0.10 & $-.10(.33)$ & $0.90[0.48,1.71]$ \\
\hline Sexual Total & 0.22 & $-.09(.18)$ & $0.92[0.64,1.31]$ \\
\hline Violent Total & 0.87 & $.17(.18)$ & $1.19[0.79,1.38]$ \\
\hline Violent + Contact Sexual & 0.08 & $.04(.14)$ & $1.04[0.79,1.38]$ \\
\hline \multicolumn{4}{|l|}{$\begin{array}{l}\text { Female Hebephilia } \\
\text { Index }\end{array}$} \\
\hline Sexual Contact & 0.00 & $-.01(.15)$ & $1.00[0.75,1.33]$ \\
\hline Sexual Non-Contact & $5.74 *$ & $.62(.26)$ & $1.86[1.17,3.11]$ \\
\hline Sexual Total & 0.50 & $.13(.08)$ & $1.14[0.98,1.34]$ \\
\hline Violent Total & $5.04 *$ & $-.30(.14)$ & $0.74[0.57,0.96]$ \\
\hline Violent + Contact Sexual & 1.27 & $-.12(.11)$ & $0.89[0.72,1.09]$ \\
\hline \multicolumn{4}{|l|}{$\begin{array}{l}\text { Male Hebephilic Self- } \\
\text { Reported Interest }\end{array}$} \\
\hline Sexual Contact & 0.01 & $.01(.16)$ & $1.01[0.74,1.38]$ \\
\hline Sexual Non-Contact & $8.95 * *$ & $.40(.15)$ & $1.50[1.12,1.99]$ \\
\hline Sexual Total & 2.31 & $.17(.11)$ & $1.19[.95,1.49]$ \\
\hline Violent Total & 0.22 & $-.08(.16)$ & $0.93[0.67,1.28]$ \\
\hline Violent + Contact Sexual & 0.18 & $-.05(.13)$ & $0.95[0.74,1.21]$ \\
\hline \multicolumn{4}{|l|}{$\begin{array}{l}\text { Female Hebephilic Self- } \\
\text { Reported Interest }\end{array}$} \\
\hline Sexual Contact & 0.20 & $-.02(.11)$ & $0.98[0.79,1.23]$ \\
\hline
\end{tabular}




\begin{tabular}{llcl} 
Sexual Non-Contact & $5.98^{* *}$ & $.32(.14)$ & $1.40[1.06,1.80]$ \\
Sexual Total & 0.77 & $.08(.09)$ & $1.09[0.90,1.30]$ \\
Violent Total & $5.72^{*}$ & $-.35(.15)$ & $0.70[0.52, .95]$ \\
Violent + Contact Sexual & 1.44 & $-.11(.09)$ & $0.90[0.75,1.07]$ \\
\hline Young Teen Boys & & & \\
Sexual Contact & 1.71 & $.10(.08)$ & $1.10[0.95,1.30]$ \\
Sexual Non-Contact & 1.44 & $.14(.12)$ & $1.15[0.91,1.45]$ \\
Sexual Total & 1.97 & $.10(.07)$ & $1.11[0.96,1.28]$ \\
Violent Total & 0.25 & $-.06(.12)$ & $0.94[0.74,1.19]$ \\
Violent + Contact Sexual & 0.12 & $.02(.07)$ & $1.02[0.89,1.18]$ \\
\hline Young Teen Girls & & $.07(.07)$ & $1.07[0.94,1.22]$ \\
Sexual Contact & 1.00 & $.20(.07)$ & $1.22[1.06,1.41]$ \\
Sexual Non-Contact & $8.97 * *$ & $.06(.06)$ & $1.06[0.94,1.20]$ \\
Sexual Total & 0.89 & $-.24(.13)$ & $0.78[0.61,1.01]$ \\
Violent Total & 3.50 & $-.04(.06)$ & $0.96[0.85,1.09]$ \\
Violent + Contact Sexual & 0.39 & & \\
\hline
\end{tabular}

Note. Results are grouped by indicator and each column represents a separate Cox regression. $* * * p<.001, * * p<.01, * p<.05$

Results by gender for pedophilia. For the male pedophilic index (see Table 22) none of the results were significant. For the female pedophilic index the only significant positive association was with sexual non-contact recidivism. Further, violent recidivism was negatively associated with higher scores on the female pedophilia index. For the male pedophilic selfreported sexual interest there was a significant association with sexual non-contact recidivism and sexual total recidivism. None of the results were significant for female self-reported 
pedophilic sexual interest. Finally, for sexual behaviour, there was an association with sexual contact and total sexual recidivism and a higher number of child boy victims. There was no significant association with sexual non-contact recidivism. For girl child victims there was no association with any of the recidivism variables. All of the effect sizes were small.

Table 22 .

Cox regressions for Pedophilia Separated by Gender

\begin{tabular}{|c|c|c|c|}
\hline Indicator & $\chi^{2}$ & B (SE) & Hazard Ratio [95\%CI] \\
\hline \multicolumn{4}{|l|}{ Male Pedophilia Index } \\
\hline Sexual Contact & 0.01 & $.23(.18)$ & $1.26[0.89,1.79]$ \\
\hline Sexual Non-Contact & 0.80 & $.08(.29)$ & $1.09[0.61,1.92]$ \\
\hline Sexual Total & 1.13 & . $17(.16)$ & $1.19[0.86,1.64]$ \\
\hline Violent Total & 2.67 & $.27(.16)$ & $1.30[0.95,1.79]$ \\
\hline Violent + Contact Sexual & 2.89 & $.22(.13)$ & $1.24[0.97,1.60]$ \\
\hline \multicolumn{4}{|l|}{ Female Pedophilia Index } \\
\hline Sexual Contact & 0.02 & $-.02(.13)$ & $0.96[0.76,1.26]$ \\
\hline Sexual Non-Contact & $5.95^{*}$ & $.52(.22)$ & $1.69[1.11,2.58]$ \\
\hline Sexual Total & 1.02 & $.12(.12)$ & $1.13[0.89,1.42]$ \\
\hline Violent Total & $4.77 *$ & $-.26(.12)$ & $0.77[0.61,0.97]$ \\
\hline Violent + Contact Sexual & 1.82 & $-.13(.09)$ & $0.88[0.73,1.06]$ \\
\hline \multicolumn{4}{|l|}{$\begin{array}{l}\text { Male Pedophilic Self- } \\
\text { Reported Interest }\end{array}$} \\
\hline Sexual Contact & 2.11 & $.27(.19)$ & $1.30[0.90,1.88]$ \\
\hline Sexual Non-Contact & $8.26 * *$ & $.52(.20)^{* *}$ & $1.68[1.14,2.49]$ \\
\hline Sexual Total & $6.76^{* *}$ & $.37(.15)^{*}$ & $1.45[1.08,1.93]$ \\
\hline Violent Total & 0.05 & $.05(.22)$ & $1.05[0.68,1.61]$ \\
\hline
\end{tabular}




\section{Female Pedophilic Self- Reported Interest}

Violent + Contact Sexual 1.09

$.17(.16)$

$1.18[0.86,1.62]$

\begin{tabular}{|c|c|c|c|}
\hline Sexual Contact & 0.62 & $-.19(.24)$ & $0.83[0.52,1.33]$ \\
\hline Sexual Non-Contact & $1.43 *$ & $.32(.14)$ & $1.40[1.06,1.80]$ \\
\hline Sexual Total & 0.10 & $.05(.76)$ & $1.05[0.77,1.43]$ \\
\hline Violent Total & 1.67 & $-.34(.27)$ & $0.71[0.42,1.21]$ \\
\hline Violent + Contact Sexual & 1.65 & $-.22(.18)$ & $0.80[0.57,1.13]$ \\
\hline \multicolumn{4}{|l|}{ Male Child Victims } \\
\hline Sexual Contact & $5.31 *$ & $.17(.08)^{*}$ & $1.19[1.02,1.38]$ \\
\hline Sexual Non-Contact & 0.08 & $.05(.17)$ & $1.05[0.76,1.45]$ \\
\hline Sexual Total & $6.76^{* *}$ & $.18(.07)^{* *}$ & $1.19[1.04,1.37]$ \\
\hline Violent Total & 0.77 & $-.12(.14)$ & $0.89[0.67,1.16]$ \\
\hline Violent + Contact Sexual & 1.20 & $.08(.07)$ & $1.08[0.94,1.25]$ \\
\hline \multicolumn{4}{|l|}{ Female Child Victims } \\
\hline Sexual Contact & 0.03 & $.02(.08)$ & $1.02[0.86,1.19]$ \\
\hline Sexual Non-Contact & 0.04 & $-.03(.15)$ & $0.97[0.72,1.30]$ \\
\hline Sexual Total & 0.04 & $-.02(.08)$ & $0.98[0.84,1.15]$ \\
\hline Violent Total & 0.42 & $.05(.07)$ & $1.05[0.91,1.20]$ \\
\hline Violent + Contact Sexual & 0.15 & $.02(.06)$ & $1.02[0.91,1.14]$ \\
\hline
\end{tabular}

Note. Results are grouped by indicator and each column represents a separate Cox regression.

$* * * p<.001, * * p<.01, * p<.05$

\section{Predictive Validity when Controlling for S99R}

A series of Cox regressions were conducted to examine whether the pedophilia or hebephilia indices predicted recidivism above and beyond the Static-99R. For these analyses, the 
Static-99R score was entered in the first step and the hebephilia (see Table 23) or pedophilia index (see Table 24) was entered in the second step. As expected, the Static-99R was significantly associated with a higher level of recidivism for all recidivism outcomes, including sexual and non-sexual outcomes. Both the pedophilia and hebephilia index did not add above and beyond the Static-99R in predicting sexual recidivism and violent (including sexual contact) recidivism. Interestingly, both the pedophilia and hebephilia indices added negative predictive power above and beyond the Static-99R to the prediction of violent only recidivism. All effect sizes were small for the analyses.

Table 23

Cox regressions for Hebephilia controlling for S99R

\begin{tabular}{lll}
\hline Indicator & B (SE B) & Hazard Ratio \\
& {$[95 \% \mathrm{CI}]$} & $\chi^{2}$
\end{tabular}

Sexual Contact

Block 1

$19.93^{* * * *}$

S99R $.19(.04)^{* * *} \quad 1.21[1.11,1.30]$

Block 2 0.97

S99R $.19(.04)^{* * *}$ $1.21[1.12,1.31]$

Hebephilia index $-.13(.13)$ $0.88[0.68,1.14]$

Sexual non-contact

Block 1

$27.55^{* * * *}$

S99R $.33(.06)^{* * *}$ $1.39[1.24,1.57]$

Block 2

S99R $.33(.06)$ $1.39[1.24,1.57]$

Hebephilia index $.29(.20)$ $1.34[0.90,2.00]$ 


\begin{tabular}{lccc}
\hline Sexual Total & & & \\
Block 1 & & & $38.87^{* * * *}$ \\
S99R & $.23(.04)^{* * *}$ & $1.26[1.18,1.34]$ & \\
Block 2 & & & 0.21 \\
S99R & $.23(.04)^{* * *}$ & $1.26[1.18,1.34]$ & \\
Hebephilia index & $-.05(.22)$ & $0.95[0.76,1.19]$ &
\end{tabular}

\begin{tabular}{llll}
\hline Violent Total & & & \\
Block 1 & & & $47.48^{* * * *}$ \\
S99R & $.26(.04)^{* * *}$ & $1.29[1.21,1.39]$ & \\
Block 2 & & & $11.00^{* * *}$ \\
S99R & $.26(.04)^{* * *}$ & $1.29[1.21,1.39]$ & \\
Hebephilia index & $-.39(.12)^{* *}$ & $0.68[0.54,0.85]$ &
\end{tabular}

Violent (including

sexual contact)

Block 1

$43.72 * * *$

S99R

Block 2

$.20(.03)^{* * *} \quad 1.22[1.15,1.29]$

S99R

Hebephilia index

$.20(.03)^{* * *} \quad 1.23[1.16,1.30]$

$-.23(.09)^{*} \quad 0.94[0.76,1.16]$

Note. Each column represents a separate Cox regression. The phallometric index was entered on the first step and the Static-99R score was entered on the second step.

$* * * p<.001, * * p<.01, * p<.05$ 
Table 24.

Cox regressions for Pedophilia Index controlling for S99R

\begin{tabular}{|c|c|c|c|}
\hline Indicator & $\mathrm{B}(\mathrm{SE} B)$ & $\begin{array}{l}\text { Hazard Ratio } \\
{[95 \% \mathrm{CI}]}\end{array}$ & $\chi^{2}$ \\
\hline \multicolumn{4}{|l|}{ Sexual Contact } \\
\hline Block 1 & & & $19.93 * * *$ \\
\hline S99R & $.19(.04)^{* * *}$ & $1.21[1.13,1.30]$ & \\
\hline Block 2 & $\mathbb{I}$ & & 0.87 \\
\hline S99R & $.19(.04)^{* * *}$ & $1.21[1.13,1.30]$ & \\
\hline Pedophilia index & $-.02(.11)$ & $0.98[0.79,1.23]$ & \\
\hline \multicolumn{4}{|l|}{ Sexual non-contact } \\
\hline Block 1 & & & $27.55 * * *$ \\
\hline S99R & $.33(.06)^{* * *}$ & $1.39[1.24,1.57]$ & \\
\hline Block 2 & & & 1.54 \\
\hline S99R & $.33(.06)^{* * *}$ & $1.38[1.23,1.55]$ & \\
\hline Pedophilia index & $.21(.17)$ & $1.24[0.88,1.72]$ & \\
\hline
\end{tabular}

Sexual Total

Block 1

$38.87 * * *$

S99R

$.23(.04)$

$1.26[1.18,1.35]$

Block 2

0.17

S99R

$.23(.04)$

$1.26[1.18,1.35]$

Pedophilia index

$.04(.10)$

$1.04[0.86,1.27]$

Violent Total

Block 1

47.48***

S99R

$.26(.04)^{* * *}$

$1.29[1.21,1.39]$ 


\begin{tabular}{lccc}
\hline Block 2 & & & $16.59^{* * *}$ \\
S99R & $.27(.04)^{* * *}$ & $1.30[1.22,1.40]$ & \\
Pedophilia index & $-.42(.11)^{* * *}$ & $0.65[0.53,0.81]$ & \\
\hline $\begin{array}{l}\text { Violent (including } \\
\text { sexual contact) }\end{array}$ & & & \\
Block 1 & & & $43.72^{* * *}$ \\
S99R & $.20(.03)^{* * *}$ & $.1 .22[1.15,1.29]$ & \\
Block 2 & & & $4.99^{*}$ \\
S99R & $.20(.03)^{* * *}$ & $0.96[0.95,0.98]$ & \\
Pedophilia index & $-.19(.08)^{*}$ & $0.83[0.71,0.98]$ & \\
& & \\
\hline
\end{tabular}

Note. Each column represents a separate Cox regression. The phallometric index was entered on the first step and the Static-99R score was entered on the second step.

$* * * p<.001, * * p<.01, * p<.05$

\section{Discussion}

In summary, ROC curve analyses suggested that hebephilia was predictive of sexual noncontact recidivism, but only when the offender admitted to the sexual interest. When controlling for opportunity to reoffend, hebephilia was consistently associated with an increased risk for sexual non-contact recidivism, but the effects were small. Interestingly, upon further examination, it was only sexual interest in pubescent girls that contributed to this relationship. A similar pattern emerged in relation to pedophilia, where pedophilia was associated with higher levels of sexual non-contact recidivism. When examined separately by gender, sexual arousal to prepubescent girls and self-reported pedophilic interest (both girls and boys) was associated with increased risk for sexual non-contact recidivism. The only variable to predict sexual contact and 
total sexual recidivism rates was having a higher number of boy child victims, but only after controlling for street time. All of these effects were small, with the exception of a medium effect for the association between the pedophilia index and sexual non-contact recidivism. Finally, both the pedophilia and hebephilia indices were not associated with sexual recidivism above baseline risk levels; however, they added negative predictive power in the prediction of violent recidivism.

\section{Sexual Interest in Children and Recidivism}

The present results were surprising, given meta-analyses that highlight the important role of sexual interest in children in the prediction of sexual recidivism (e.g., Hanson \& MortonBourgon, 2005). Despite previous meta-analytic findings, there has been individual variation across studies that have looked at pedophilia and its relationship with sexual recidivism (e.g., Wilson et al., 2011; Quinsey et al., 1995). Further, there have been fewer studies that have examined hebephilia, but both found support for this predictor in a sample of contact sexual offenders (Canales et al., 2009; Proulx et al., 1997). It is important to note that the studies that found this relationship have examined hebephilia in relatively homogenous samples of high-risk offenders serving a sentence in a federal penitentiary. Although discrepancies in individual studies would not be unexpected due to sampling error, it could suggest there are important moderators that need to be taken into account when considering whether sexual interest in children is predictive of sexual recidivism.

One potential explanation for the present results comes from studies that have emerged from a hospital clinic similar to the present sample (e.g., Moulden et al., 2009). The sample in these past studies come from a large Sexual Behaviour Clinic in Canada with a heterogeneous population, as opposed to samples comprised of federal offenders or other high-risk samples, 
where there is a selection bias. In studies with a more heterogeneous sample, researchers found that the pedophilia assault index (i.e., greater response to sexual stimuli depicting both children and sexual violence, relative to response to sexually violent stimuli involving adults), but not the pedophilia index, predicted sexual recidivism (Firestone et al., 2000; Moulden et al., 2009). This is crucial as it suggests that it may potentially be the co-occurrence of sexual sadism or a sexual interest in coercive sex (i.e., biastophilia) and sexual interest in children that might be important in the prediction of sexual recidivism. As such, it may be the inclusion of assault stimuli in depictions of children during phallometric testing that predicts recidivism in samples of relatively low risk offenders. Unfortunately, there is no way to examine this in the present study as the stimuli were only designed to assess sexual interest in prepubescent or pubescent children and the audiotaped stimuli were specific to encounters with children that were not accompanied by force.

Methodological issues also need to be considered as factors contributing to the results. First, the sample was on average 41 years of age at the time of the assessment (i.e., the start of the follow-up period). A well-established finding in the criminology literature is that criminality decreases across the lifespan (e.g., Farrington, 1986). Further, for older sexual offenders decreased levels of testosterone would contribute to reductions in sexual arousal and concurrent sexual functioning problems. This would in turn contribute to lower rates of sexual recidivism (Barbaree \& Blanchard, 2008). Thus, the average age prior to the start of the follow-up periods suggests that these offenders would likely be at a lower risk to reoffend during the follow-up period. Further, with a Static-99R score of 2 (average score in the present sample was 1.75), approximately $12 \%$ of sexual offenders in the Static-99R normative sample reoffended sexually within a ten-year period (Phenix, Helmus, \& Hanson, 2012). Power was calculated based on an 
expected recidivism rate of $20 \%$; however, recidivism rates were more in line with the estimates provided in the Static-99R manual for a non-routine (i.e., preselected for treatment) sample. As such, it is likely that the present study was underpowered. Nonetheless, this does not explain that sexual interest in children was associated with sexual non-contact recidivism, which had the lowest base rate. Another methodological consideration is that for some offenders in the sample there was a considerable period of time that passed between their index offence and the assessment date. As such, for some offenders they were already risk free in the community for a long period of time prior to the assessment, which marked the start of the follow-up period. This is important as the longer one is risk free in the community the less likely they are to recidivate (Harris \& Rice, 2007).

Finally, many of these offenders went through psychological treatment with some receiving sex drive reducing medication, in addition the psychological treatment. The research literature has suggested a small but significant effect for psychological and pharmacological treatment on reducing sexual recidivism (e.g., Hanson et al., 2000). It is also possible that the higher risk offenders (arguably those with paraphilias) were more closely monitored by community agencies, such as probation and parole offices. Management strategies of high risk offenders have been shown to have an impact on reducing recidivism (e.g., Circles of Support and Accountability; e.g., Duwe, 2013). Unfortunately, these factors were not coded in the present study as they were not consistently recorded in clinical files. The impact of clinical management of sexual offenders with paraphilias on recidivism rates is an important area of inquiry and should be explored in future research.

The only association with sexual contact recidivism was for behavioural indicators of pedophilia (i.e., sexual offending against boys; see Seto \& Lalumiére, 2001). Specifically, a 
greater number of boy child victims was associated with sexual contact recidivism and selfreported sexual interest in prepubescent boys was associated with sexual recidivism overall. This finding is consistent with the literature which has found an association between boy child victims and/or a sexual interest in prepubescent boys and recidivism (e.g., Hanson \& Bussiére, 1998).

The present results highlight the importance of pedophilia and hebephilia in the prediction of non-contact sexual recidivism. Given the low base rate of sexual non-contact recidivism, it was surprising that the various indicators only predicted sexual non-contact recidivism. The importance of sexual interest in children in the prediction of sexual non-contact recidivism was replicated across the various indicators adding further credence to the results. It is of note that many men who were assessed at CAMH were non-contact offenders only (e.g., exhibitionism, voyeurism). It was not possible to eliminate sexual offenders with only noncontact offences (with the exception of child pornography only offenders) or provide an estimate for the number of them in the sample, as non-contact victims were included in official victim counts in the pre-existing database. Therefore, the prediction of sexual non-contact recidivism could partially be explained by the inclusion of non-contact sexual offenders, as non-contact offending history may predict future non-contact sexual offending, but not future contact sexual offending.

Interestingly it was sexual interest in prepubescent and pubescent girls as opposed to prepubescent or pubescent boys, which emerged as important in predicting sexual non-contact recidivism. These findings suggest that there is something unique about the contribution of sexual interest in prepubescent and pubescent girls in the prediction of non-contact sexual recidivism. In the present study, the majority of non-contact sexual recidivism constituted child 
pornography or child luring cases. Child pornography is often viewed as highly consistent with sexual interests (Seto, Cantor, \& Blanchard, 2006). As the majority of child pornography depicts female children, this may suggest that the individuals who view it have a sexual interest in young girls (Seto, 2013). These results speak to the important of considering sexual contact and sexual non-contact recidivism separately in studies of sexual recidivism. Further, they add to existing research that signifies the importance of sexual interest in pubescent children as a risk factor for non-contact sexual recidivism (e.g., Eke \& Seto, 2011; Seto, \& Eke, 2015).

\section{Implications}

Clinical Sexology. The present results need to be interpreted within the wider debate about the validity of hebephilia as a construct. There has been considerable debate about hebephilia since Blanchard and colleagues (2009) concluded that the diagnosis of pedophilia should be expanded to include hebephilia. The present results suggest that hebephilia has predictive validity for sexual non-contact recidivism after taking opportunity to reoffend into account. It is important to note that despite these findings, two previous independent research teams have found that the hebephilia index was predictive of sexual recidivism, adding some further credence to the argument that hebephilia has predictive validity (Canales et al., 2009; Proulx et al., 1997).

Given the high degree of overlap between pedophilia and hebephilia (discussed in $\underline{\text { Study }}$ $\underline{1}$ and Study 2), it was not surprising that both pedophilia and hebephilia have a similar pattern in

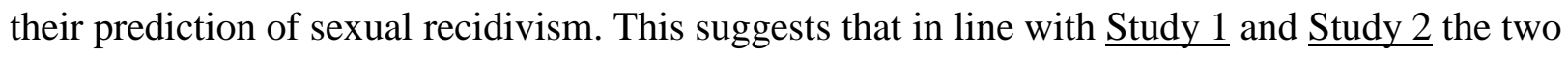
constructs have similar correlates, which has also been found in other research that has looked at differences in cognitive functioning, neuroimaging, and developmental history (e.g., Blanchard et al., 2002, Cantor et al., 2004, 2005, 2006, 2008, 2012). As such, the argument by Blanchard 
and colleagues (2009) that pedophilia should be extended to include hebephilia is consistent with the research, especially due to the high degree of overlap between pedophilia and hebephilia and their similar correlates.

Clinical Forensic Psychology. Just as clinicians consider pedophilia during the assessment of sexual offenders, it is also important to consider hebephilia, given its predictive validity in relation to sexual non-contact recidivism. Nonetheless, even if the present results did not suggest a relationship between hebephilia and sexual non-contact recidivism, it is still important for clinicians to consider it during a comprehensive assessment for two reasons. First, hebephilia has been found to be associated with a greater number of child and teen victims and offenders could likely not act on this sexual interest in a legal manner (see Study 2). As such, offenders may benefit from treatment programs that teach them how to manage sexual interest in pubescent children so that they do not commit a sexual offence by acting on their sexual interest. There is emerging evidence from Germany that men with sexual interest in prepubescent or pubescent children are distressed by this sexual interest and motivated to seek treatment in order to curb their sexual interests and reduce their risk to the community (Beier et al., 2009). Thus, it may be an appropriate treatment target that could also impact rates of non-contract sexual recidivism. Finally, previous studies have found a relationship between hebephilia and overall rates of sexual recidivism (Canales et al., 2009; Eke \& Seto, 2011; Proulx et al., 1997), which suggests that it may be an important risk factor.

These results should not be interpreted to suggest that it is not important to consider pedophilia in risk assessment of sexual offenders, as there is robust meta-analytic data to suggest it is an important risk factor (Hanson \& Morton-Bourgon, 2005; Mann et al., 2010). The present results suggest that both pedophilia and hebephilia are important to consider as they are 
associated with higher rates of sexual non-contact recidivism and that behavioural indicators of sexual interest in pubescent boys predicts sexual contact and total sexual recidivism. Although non-contact sexual recidivism is arguably of lesser concern than risk for contact sexual recidivism (i.e., does the offender repeat the same behaviour or escalate his offending), noncontact sexual recidivism still contributes to significant harm and societal concern. Further, it is of increasing interest among professionals interested in the prediction of risk in non-contact sexual offenders and the best way to effectively intervene with this population. Additionally, it was found that sexual interest in boy prepubescent children and a greater number of boy child victims was associated with overall rates of sexual recidivism. Taken with the extant literature in the area this suggests there is considerable support for considering pedophilia in the assessment of risk to the community. Additionally, there is support for the consideration of hebephilia in relation to sexual non-contact recidivism, and based on past research likely contact sexual recidivism (e.g., Canales et al., 2009; Proulx et al., 1997).

\section{Limitations and Future Research Directions}

Prior to discussing future research directions, there are a number of limitations that should be considered when interpreting these findings. First, the present study relied entirely on official records in order to code recidivism, which are subject to the large dark figure of sexual crime. As such, these official records underestimate the true nature of sexual recidivism and sexual crime in general. Further, when coding recidivism from criminal records it was not clear from the entry what truly motivated the offence. For example, when coding some of the records for priors it became apparent that voyeurism would often appear on records as trespassing by night; however, when coding from official files there was no way to know whether ambiguous offences that occurred after the assessment date (i.e., assessment) were sexual in nature. Thus, 
they were entered based on the offence name regardless of whether the coders believed they were sexual in nature. Finally, recidivism in this study was examined as a binary outcome. Although this is common in the recidivism literature, this does not speak to issues pertaining to escalation, chronicity, frequency, the nature of the offender's past criminal career and severity. For example, do offenders continue to offend at the same level of severity, or does it escalate over time? These types of questions are important to consider and can only be answered through future research on recidivism with a more nuanced understanding of the criminal trajectories of sexual offenders (Lussier \& Davies, 2011).

Future research should clarify the role of hebephilia in sexual recidivism and consider important moderators of this relationship (e.g., inclusion of assault stimuli in phallometric data). In the present study a number of indicators of hebephilia were considered. Nonetheless, the authors did not have access to official diagnostic information (i.e., how the clinician interpreted this information to arrive at a diagnosis) and this should be considered in future research. Further, the questions of whether the consideration of other paraphilias in addition to hebephilia improves predictive accuracy should be addressed in future research. This is particularly important when one considers the considerable overlap between pedophilia and hebephilia. It is possible that those who show sexual interest in prepubescent and pubescent children would be a higher risk to reoffend as they would have greater opportunity given a wider potential victim pool. Finally, previous studies have found that the combination of antisociality and deviant sexual interest predict sexual recidivism (e.g., Hildebrand, de Ruiter, \& de Vogel, 2004). In the present study antisociality was not specifically assessed; however, it is possible that in combination with hebephilia it would be predictive of recidivism. 


\section{Chapter 5}

\section{Implications}

\section{Summary}

In summary, the dissertation examined erotic-age interests with a specific focus on the validity of hebephilia. Study 1 examined convergent validity expanding on previous research by including those who deny they have a sexual interest in children. Despite their exclusion in past research, results were consistent with research in the area (Beier et al., 2009, 2013; Blanchard, Lykins et al., 2009) that suggests there exist a group of men who are sexually interested in pubescent children as evidenced by phallometric responding, self-report, and sexual behaviour. Further, there is a high degree of non-exclusivity between pedophilia and hebephilia. Study 2 examined the relationship between hebephilia and sexual offending victim choice in order to establish concurrent validity. Both pedophilia and hebephilia had similar sexual victim age choice correlates and were associated with having a greater number of victims under the age 15 . Although both pedophilia and hebephilia were associated with a greater likelihood of victim age polymorphism, an examination of the standardized betas suggest that hebephiles were less age specific. Finally, Study 3 examined the predictive validity of hebephilia in sexual recidivism and found that like pedophilia, hebephilia was associated with an increased risk for sexual noncontact recidivism, but not sexual contact recidivism. Throughout the studies, pedophiles and hebephiles were often more similar than they were different, but hebephiles often differed from teleiophiles.

Despite the many similarities, there were also differences that emerged between pedophilia and hebephilia throughout the dissertation. In $\underline{\text { Study } 1}$, there were a number of differences in sexual arousal to the various phallometric categories. For example, hebephiles had 
a significantly higher response to pubescent children compared to pedophiles. In $\underline{\text { Study } 2,}$ hebephilia was more consistently found to be associated with victim age polymorphism than pedophilia. Finally, in $\underline{\text { Study } 3}$ behavioural indicator of pedophilia (i.e., greater number of boy child victims) were associated with an increased risk for sexual contact and total sexual recidivism, whereas indicators of hebephilia only predicted sexual non-contact recidivism. Despite the differences that emerged throughout the three studies, these differences were largely overshadowed by the high degree of overlap between the two constructs and their similar correlates.

Taken together the consistent theme throughout this dissertation is that both pedophilia and hebephilia are more similar than they are different. This is consistent with research on the neurodevelopmental perturbation hypothesis (see below) that has found a similar pattern of results for both pedophilia and hebephilia (e.g., Cantor et al., 2004, 2005, 2008, 2012). Based on past research and the results of the present dissertation, it is argued that pedophilia and hebephilia are overlapping constructs. This conclusion has important implications for both the field of clinical sexology and clinical forensic psychology.

\section{Implications for Clinical Sexology}

Chapter 1 began with an examination of the construct of hebephilia and briefly reviewed the debate that started after Blanchard, Lykins, and colleagues (2009) proposed that pedophilia be expanded to include hebephilia. Briefly, they recommended that pedophilia be renamed pedohebephilic disorder with the inclusion of specifier that would indicate the focus of the erotic-age interest (i.e., pedophilic type, hebephilic type, and pedohebephilic type). Both the extant literature and the results of the dissertation suggest that this initial proposal for the DSM is appropriate and consistent with research in the area. 
If pedophilia continues to be included as a mental health diagnosis, hebephilia should also be explicitly included in future revisions of the DSM and ICD. Given the high degree of overlap between the two, the conclusion of Blanchard, Lykins, and colleagues (2009) to rename the disorder Pedohebephilia with specifiers for the focus of erotic-age interest would be the most appropriate way to include hebephilia in future diagnostic manuals. The conclusion that hebephilia be included alongside pedophilia in diagnostic nomenclature is supported by two main findings from the dissertation. First, various aspects of validity were examined and hebephilia was found to have convergent, concurrent, and predictive validity; however, in some cases this was not as clearly demonstrated. This is an important finding given that some have argued that hebephilia is an invalid construct developed for unethical purposes (e.g., Franklin, 2009, 2010). Second, hebephilia and pedophilia are overlapping constructs with similar correlates, which suggest it is hard to consider one without consideration of the other. The pattern of similarities in the present dissertation is not unique and has been found in research on the neurodevelopmental hypothesis of pedophilia where both pedophilia and hebephilia have been suggested to have a similar etiology (Cantor et al., 2004, 2005, 2008; 2012).

Nonetheless whether hebephilia is maladaptive was not specifically addressed, which serves as a point of contention in the literature (e.g., Franklin, 2009, 2010; Green, 2010; Janessen, 2009; Rind \& Yuill, 2012). Many critiques of hebephilia have suggested that it is based on a North American view of acceptable sexual partners and that in many cultures men have sexual relations with pubescent children. In fact, according to these researchers hebephilia might have been evolutionarily adaptive (Franklin, 2009; Janssen, 2009; Green, 2010). It is of note that similar arguments have been made in relation to pedophilia (e.g., Green, 2002). Despite these arguments, recent reviews of the anthropological literature suggest that, even in those cultures 
where marriage between adults and pubescent children is permitted, it is often an economic or social consideration and sexual contact does not occur right away (Hames \& Blanchard, 2012; Ryniker, 2012). Further, there is evidence to suggest that men are more attracted to signs of physical maturity that are more consistent with Tanner Stage 5 of sexual development than signs of sexual immaturity (Buss, 1994; Kenrick \& Keefe, 1996). Despite these findings, the debate continues in the literature on whether hebephilia is evolutionarily adaptive. This is perhaps one of the more difficult areas to address and reach a consensus on in the literature.

A related issue is whether hebephilia would meet the threshold of a mental health disorder. The current diagnostic nomenclature is guided by an instrumental paper by Wakefield (1992). Wakefield argued that a condition is a mental disorder if it meets the criteria of harmful dysfunction. Specifically, harmful dysfunction “exists when the failure of a person's internal mechanisms to perform their functions as designed by nature impinges harmfully on the person's well-being as defined by social values and meaning" (p. 373). Thus the determination of whether a condition is a mental disorder requires two conditions. First and foremost there needs to be some type of dysfunction based on faulty internal mechanisms and second this dysfunction must have a negative impact on the individual's functioning. Given that this diagnosis guides the DSM and related diagnostic manuals, it is crucial to briefly discuss whether hebephilia would meet the criterion of harmful dysfunction as proposed by Wakefield (1992). It is of note that Wakefield (2012) argued that hebephilia should not be included in the DSM. Nonetheless, this argument was based on general confusion about the meaning of the term, a disregard for the wider literature on hebephilia, and confusion about the distinction between hebephilia and ephebophilia. 
As such, a more careful application of Wakefield's (1992) concept of harmful dysfunction is warranted. Perhaps the more contentious issue is whether hebephilia is dysfunctional, which is somewhat related to arguments pertaining to whether it is adaptive. Regardless of this debate, it is important to note that according to Wakefield (1992), a purely evolutionary argument is insufficient in and of itself in determining whether a condition meets the threshold to be a mental disorder. For example, Wakefield (1992) argued that even if a condition results in decreased reproductive benefits, this does not necessarily mean it has an impact on that person's well-being or functioning. Further, there are conditions that can result in serious harm, but do not impact reproductive functioning or evolutionary survival.

Therefore, it is crucial to set aside the debate on whether hebephilia is evolutionarily adaptive when considering whether hebephilia is a mental disorder. Instead of examining hebephilia from an evolutionary standpoint, it is more useful to examine it from the standpoint of etiology in order to assess the criterion of dysfunction. James Cantor and colleagues have spent the last decade conducting research on the neurodevelopmental perturbation hypothesis of pedophilia and hebephilia. This hypothesis was put forth by Cantor and colleagues (2002) in an empirical paper and argued that something goes awry during neurodevelopment that directly influences the development of sexual interest in children alongside other cognitive, physical, and behavioural deficits (Blanchard et al., 2002). There has been support for this neurodevelopmental dysfunction that has emerged from studies analyzing cognitive functioning (e.g., intelligence; Cantor et al., 2004; Suchy, Whittaker, Strassberg, Eastvold, 2009), physical markers (e.g., handedness; Cantor et al., 2005), and behavioural indicators (e.g., higher likelihood of head injuries prior to the age of 13; Blanchard et al., 2002). Further, recent neuroimaging research has suggested there are white matter differences in the brains of pedophiles and hebephiles that may 
influence the development of atypical sexual interest (e.g., Cantor et al., 2008, 2012). Thus, from a biological standpoint there is emerging support for the argument that both pedophilia and hebephilia would meet Wakefield's dysfunction criterion.

The second part of Wakefield's definition of a mental disorder is that the dysfunction results in harm to the individual. It is important to note that there are men with sexual interest in prepubescent and pubescent children who do not report distress or impairment from their behaviour. In fact, some of these men have gone so far as to argue that children can consent to sexual activity, and society is the problem, as they prohibit sexual contact between adults and children. Further, there are men with pedophilia and hebephilia who do not act on their sexual interest, which is why the distinction between sexual interest in children and sexual offending is crucial. This is where the distinction in DSM-5 between a paraphilia and a paraphilic disorder is of paramount importance. If the individual does not experience distress or impairment, the person would be said to have a paraphilia, but it would not be enough to classify that individual as having a paraphilic disorder.

Even if the individual is not distressed by his sexual interest, in cases where one acts on this sexual interest it would be associated with significant harm to the victim. It is of note that there are other disorders in the diagnostic nomenclature where others would be more likely to experience harm as a direct result of the conduct of the individual, which could result in legal sanctions (e.g., Antisocial Personality Disorder). Nevertheless, in cases where the individual is acting on their sexual interest in pubescent children, there could be resulting legal implications that would have negative impact on the offender's wellbeing. Nonetheless, the fact that they victimize others should not serve as the basis of the harm criterion; however, fear of getting caught and its associated negative consequences and/or persistent anxiety could meet the harm 
criterion. As previously discussed, pubescent children would likely be under the age of consent and acting on this sexual interest would have serious legal implications, such as incarceration. Even if the individual was not caught they may have significant levels of anxiety about the negative consequences associated with their sexual interest, which would qualify as harm. As such, it would be difficult to make the argument that having a sexual interest in prepubescent or pubescent children would not have a negative impact on the individual. Further, a prevention program in Germany that recruits pedophiles and hebephiles for free treatment has found that both pedophiles and hebephiles experience genuine distress about their sexual interest (Beier et al., 2009, 2013). As such, hebephilia could be viewed as resulting in significant harm to others if the interest was acted on, harm to self, and/or significant distress.

\section{Implications for Clinical Forensic Psychology}

The present dissertation has implications for the field of clinical forensic psychology specific to the area of sex offender assessment and management. Atypical sexual interests have been found to be important predictors of recidivism (Mann et al., 2010). In essence, predictors of recidivism are referred to as criminogenic needs and are targeted in treatment programs in order to reduce the likelihood of recidivism (Andrews et al., 1990; Andrews \& Bonta, 2010). The careful targeting of criminogenic needs in sexual offenders has been found to reduce levels of recidivism in sexual offenders (Hanson et al., 2009).

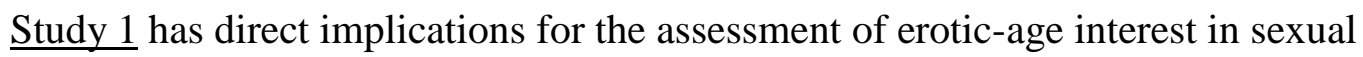
offenders. The results suggest that hebephilia can be reliably assessed through a careful and systematic assessment and that there is evidence for convergence of these measures. Just as it is paramount to assess for the presence of pedophilia in sexual offenders, it is also important to assess for the presence of hebephilia, especially given the high degree of overlap between the 
two constructs. Further, hebephilic sexual interest is associated with having a greater number of young teen victims; however, it is also associated with victim age polymorphism. This suggests that even if one has a younger child victim or an older teen victim, it is important to still inquire about sexual interest in prepubescent or pubescent children. The offence specific fallacy posits that sexual offenders confine themselves to one specific type of offence due to a specific sexual preference (Laws, 1994). This fallacy guides the study of sexual offenders influencing both the research that is conducted and the application of this research across various settings (e.g., police investigation; Laws, 1994; Robertillo \& Terry, 2007) and has the potential to be misleading. Based on these findings and similar research on victim age polymorphism, it is important to note that offenders may not always offend specific to their sexual interests. As such, it is important to assess sexual interest in children in child molesters, but also in offenders with older victims.

The assessment of sexual interest in children and other paraphilias is inherently complicated as it generally relies on accurate self-report. In both $\underline{\text { Study } 2}$ and $\underline{\text { Study } 3}$, selfreported sexual interest often times produced larger effects than sexual arousal or sexual behaviour indicators. This may lead some to question the utility of phallometric assessment. Although self-report is important, the majority of men who are sexually interested in children would be motivated to not disclose this information due to negative consequences and stigma. As such, there is a need for indirect methods of assessment, such as phallometric assessment, which has been viewed as the gold standard in the assessment of atypical sexual interests (Laws et al., 2000). Furthermore, in the assessment of sexual interest, multimodal assessment is crucial in arriving at a diagnosis (e.g., Banse et al., 2010; Laws et al., 2000). The assessment process should follow the pattern of careful assessment used with other mental health disorders. This includes a review of pertinent files, interview questions specific to sexual history and interests, 
review of victim information and police reports, questionnaires (e.g., Multiphasic Sex

Inventory), and objective measures such as phallometric assessment or other implicit assessment measures (e.g., IAT; Brown et al., 2009). Although previous research has found that convergence between measures is not necessary (Babchishin, Nunes, \& Kessous, 2014), in clinical psychology the greater the convergence across various measures the more confident one would be in rendering a diagnosis. It would seem crucial than in applied settings that evaluators look for convergence among various assessment measures, which is especially important as labelling of someone as pedophilic or hebephilic has serious consequences (e.g., increased risk rating, involvement of child protective services if children are in the home). As such, it is important to be cautious when assigning this label and avoiding false positives is likely more important than increasing the number of true positives.

Based on the results of Study 3 , it is important to address whether the assessment of pedophilia and hebephilia is important from a risk assessment standpoint. Both pedophilia and hebephilia had predictive validity in relation to sexual non-contact recidivism. Although this is arguably less serious than sexual contact recidivism, it still does suggest that sexual interest in both prepubescent and pubescent children puts one at increased risk for certain types of sexual recidivism. Further, there is considerable research to support the importance of considering pedophilia in relation to risk (e.g., Firestone et al., 2000) and to some extent hebephilia (Canales et al., 2009; Eke \& Seto, 2011; Proulx et al., 1997). Therefore, from a risk standpoint both pedophilia and hebephilia should be considered when conducting risk assessments and conceptualized as a criminogenic needs that need to be targeted appropriately in sexual offender treatment. Nonetheless, it is important to highlight that both pedophilia and hebephilia did not add incremental validity to the Static-99R in the prediction of sexual recidivism. Thus, the 
presence of pedophilia or hebephilia should not be used to adjust actuarially determined risk estimates. More specifically, clinical judgement based on whether an offender is pedophilic or hebephilic should not change the actuarial risk estimate produced by the Static-99R.

\section{Future Research Directions}

There are a number of future directions that should be pursued in order to further the research literature on hebephilia. Although this has been discussed in previous chapters, there are a few major areas that will be reiterated as important areas of future inquiry. First, it is crucial to conduct research on the impact including hebephilia in the DSM and other diagnostic manuals on diagnosis and applied practice. Similarly it is important to understand the impact explicitly including hebephilia in the $D S M$ would have on forensic practice. For example, one area of inquiry could examine the impact its inclusion would have on the number of men who are civilly committed, as a significant concern is the way the construct of hebephilia would be used in forensic practice (e.g., Wakefield, 2012)

Additionally, there is a need for more research on the etiology of both pedophilia and hebephilia. The neurodevelopmental perturbation hypothesis has been the only hypothesis that has directly addressed the etiology of hebephilia. Specifically, the overall finding is that both pedophilia and hebephilia could be due to a neurodevelopmental perturbation (e.g., Cantor et al., 2008, 2012). Nonetheless, if one considers the diathesis-stress perspective, it is possible that these biological differences serve as a predisposition for the development of sexual interest in children and various other factors influence whether this predisposition develops into a disorder. Thus, it is crucial to examine other factors in relation to the development of hebephilia and to assess whether the development of pedophilia and hebephilia is similar or differs on certain dimensions. One such factor may be childhood sexual abuse, as meta-analyses have found that 
sex offenders are more likely to experience sexual abuse than other offenders, and that those who offend against children have even higher rates of sexual abuse (Jespersen, Lalumiére, \& Seto, 2009; Seto \& Lalumiére, 2010). Further, there is some evidence to suggest there may be differences in environmental factors, as Greenberg, Bradford, and Curry (1993) found that pedophiles and hebephiles (whom they classified through sexual offending victim age choice) not only had high levels of sexual abuse, but the age they were when they were sexually abused corresponded with the age of their victims. This suggests that there may be subtle etiological differences between pedophilia and hebephilia that may impact the expression of their erotic age interest.

Future research should also directly examine whether erotic-age interest is a taxon, which has limited and conflicting research (e.g., Mackaronis et al., 2011; Schmidt et al., 2013). This research should utilize a variety of assessment tools, including phallometric assessment. This would address whether pedophiles and hebephiles are a distinct group and whether there is a strict cut-off point on these measures that would differentiate them from teleiophilie, or if the constructs exist a long a continuum. Further, researchers should examine the best way to assess non-exclusivity in erotic-age interest. It would also be important to assess whether nonexclusivity in sexual interest is meaningful from a risk and treatment perspective (e.g., are these individuals of higher risk and in need of higher intensity services).

Finally, the efficacy of treatment for hebephiles has not been examined. There is currently research underway in Germany evaluating their prevention program (e.g., Beier et al., 2009). This research can address whether treatment efficacy differs for pedophiles and hebephiles. Nonetheless, this is a highly specific prevention program with men who admit to and are motivated to seek treatment. When conducting sex offender treatment, providers are faced with 
additional complexities around high rates of denial and minimization of offending and atypical sexual interest. It remains unknown whether there are unique treatment needs for hebephiles and if they respond differently to treatment than pedophiles or teleiophiles. 


\section{Appendix A}

\section{Comparison of DSM criteria for Pedophilia}

$\begin{array}{lll}\text { Pedophilia }(D S M-I V-T R) & \text { Pedophilic Disorder (Proposed } & \text { Pedophilic Disorder } \\ & \text { for DSM-5 as of 2012) } & (D S M-5)\end{array}$

\begin{tabular}{|c|c|c|}
\hline A. Over a period of at least 6 & A. Over a period of at least 6 & A. Over a period of at \\
\hline months, recurrent, intense & months, an equal or greater & least 6 months, recurrent, \\
\hline sexually arousing fantasies, & sexual arousal from & intense sexually arousing \\
\hline sexual urges, or behaviours & prepubescent or early pubescent & fantasies, sexual urges, or \\
\hline involving sexual activity with a & children than from physically & behaviours involving \\
\hline prepubescent child or children & mature persons, as manifested by & sexual activity with a \\
\hline \multirow[t]{2}{*}{ (generally age 13 or younger) } & fantasies, urges, or behaviours. & prepubescent child or \\
\hline & & children (generally age 13 \\
\hline B. The person has acted on & B. The individual has acted on & or younger) \\
\hline these sexual urges, or the sexual & these sexual urges, or the sexual & \\
\hline urges or fantasies cause marked & urges or fantasies cause marked & B. The person has acted \\
\hline distress or interpersonal & distress or impairment in social, & on these sexual urges, or \\
\hline \multirow[t]{2}{*}{ difficulty } & occupational, or other important & the sexual urges or \\
\hline & areas of functioning. & fantasies cause marked \\
\hline C. The person is at least 16 & & distress or interpersonal \\
\hline years and at least 5 years older & C. The individual must be $a t$ & difficulty \\
\hline than the child or children in & least 18 years of age and at least & \\
\hline Criterion A. & 5 years older than the children in & C. The person is at least \\
\hline Note. Do not include an & Criterion A. & 16 years and at least 5 \\
\hline
\end{tabular}


individual in late adolescence

involved in an ongoing

relationship with a 12 - or 13 -

year-old

Specify if:

Sexually attracted to males

Sexually attracted to females

Sexually attracted to both

Specify if:

Limited to incest
Specify type:

Classic Type-Sexually

Attracted to

Prepubescent Children (Tanner

Stage 1)

Hebephilic Type-Sexually

Attracted to Early

Pubescent Children (Tanner

Stages 2-3)

Pedohebephilic Type-Sexually

Attracted to

Both

Specify types:

Exclusive type (attracted only to

children)

Nonexclusive type
Specify type:

Sexually Attracted to Males

Sexually Attracted to Females

Sexually Attracted to Both years older than the child

or children in Criterion A.

Note. Do not include an

individual in late

adolescence involved in

an ongoing relationship

with a 12- or 13-year-old

Specify whether:

Exclusive type (attracted

only to children)

Nonexclusive type

Specify if:

Sexually attracted to

males

Sexually attracted to

females

Sexually attracted to both

Specify if:

In a Controlled Environment Specify if:

In Remission (No Distress, Limited to incest

Impairment, or 
Recurring Behaviour for Five

Years and in an

Uncontrolled Environment) 
Appendix B

\section{CPIC Study Coding Manual}

\section{Principal Investigator: Skye Stephens}

January 8, 2014 


\section{Step 1: Access the database and CPIC records}

-Log in to the computer in Skye's office or the research room.

-Access the T-Drive: Complex Mental Illness-> SBC Treatment -> FMRI Study -> CPIC. In this file there will be the dataset and excel worksheet.

-In order to find where you should begin please consult the excel datasheet. You should start entering data where the last person left off. Only enter file information for those offenders with a 1 under record (this means we received a record from the RCMP). If they do not have a record you are unable to score them and should move on to the next offender in the database.

-Pull the CPIC and the file with Michael Kuban's report for the offender. Ensure that the name and DOB match on all of the information.

-You will likely need to search for the offender on CHARTMAXX in order to get more complete assessment info. CHARTMAXX search make sure you select "all" (as opposed to pre or post 2009). All of the offenders though were assessed prior to 2009 (they may have come back more than once for an assessment).

-If you are running inter-rater reliability checks please consult Appendix A.

-Do not look at phallometric results when entering the data. We want to try and remain blind to whether there is a paraphilia. This means you should only look at the top part of the report. -If you recognize the name of an individual for the CPIC study you are not to look at this individuals file and/or score them for the study. Please flag the name of this individual in the Excel worksheet and someone else will enter the information. You may move on to the next file after flagging this individual in the Excel database.

-Remember there is an FAQ document in the CPIC file folder with common questions. This will continue to be added to as we go along with data entry. 
-The purpose of scoring these files is to use CAMH assessment information to predict

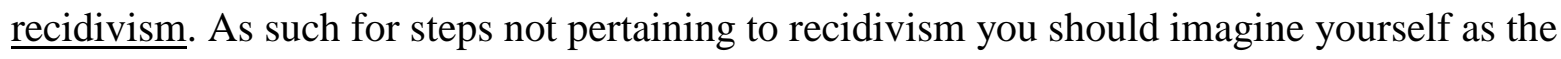
clinician on the date of the assessment when coding the information in steps 2 through 4 . It may be helpful to physically cover the CPIC after you locate the index if there is recidivism information in order not to contaminate your reading of the criminal record.

\section{Step 2: Score the Static-99R}

\section{Prior to scoring the Static-99R}

-Match the name on the file material to the name in the CPIC SPSS database. Double check it is the correct person by cross-referencing with the date of birth. Everything should be consistent before proceeding. If there is something that is not consistent do not enter this person into the dataset. Enter the problem in the excel worksheet and the PI will investigate further. At this point you can start back at step 1 after putting all file information back.

-Enter in your entry code.

Scorer:

$1=$ Skye

2= Kylie

-Enter the date of the phallometric assessment at CAMH. This is the date on the top of Michael Kuban's report (the date of the phallometric assessment). -Enter phallodate: yyyy/mos/day

-The index offence for our purposes is what resulted in their referral to CAMH (e.g., man on probation for sexual assault in 1996 comes to CAMH in 1997 the index on the record will be from 1996). The index offence will be stated in Michael's report (he usually reports this first and then goes on to report on their history of offending). Match the index offence(s) included on 
Michael's report to what is included on the individual's CPIC. If you cannot match the index offence as laid out in Michael's report to the CPIC note this in the excel datasheet and start back at step 1.

-In some cases there may be a sustained pattern of sex offending which is why the individual was referred to $\mathrm{CAMH}$. If the sustained pattern of offending led to different sentencing occasions take the latest index offence with a disposition date on the CPIC. For example, a person was assessed at CAMH in 2002 and committed a sex offence in $2000,1995,1992$. All of these offences were dealt with on separate sentencing occasions. In this case the sex offence from 2000 would be the index as it is the latest entry on the CPIC. If all of the index offences were dealt with on the same sentencing occasion (e.g.. all were dealt with in 2000) then this is an index cluster and together they comprise the index offence.

-If the individual has committed another criminal act between the index sexual offence and the assessment appointment at CAMH (e.g., drug offence while in prison) treat this as part of the index offence as we would have had this information at the time of the assessment. In other words it is not scored as recidivism.

-For the purposes of scoring the STATIC-99R the definition of an index offence only involves crimes where the individual has been charged or convicted. Sometimes you will encounter situations where the individual has only admitted offences, and there are no charges or convictions. For our purposes we cannot score the Static-99R on this individual as they are not a detected offender (leaving Static-99 items in dataset blank). The Static-99R should only be scored on detected offenders (e.g., at a minimum there was a charge associated with the index). 
The reason they were referred must have resulted in a charge or conviction in order for you to score the Static-99R.

-If they have only admitted sexual offences and there is no index offence as per the Static-99R note of this in the Excel worksheet. Skip the entry of Static-99R file information and proceed to step 4 (index victim information).

-For our purposes we can score the Static-99R on CP or non-contact sexual offenders as this is for research purposes as opposed to a clinical assessment.

\section{-Enter NatIndex:}

$0=1$ index

$1=$ index cluster

$2=$ Self-report offences only

3= No offending history

-Enter Distribution if to Immigration or it notes death

$0=$ none to report

$1=$ immigration

$2=$ death noted under distribution

-Enter distdate: Enter date of distribution for above yyyy/mos/day

-Enter the date of conviction for the index offence/index cluster as it appears on the CPIC sheet in the data set. This will appear in the first column of the CPIC under Date and Place of Disposition

-Enter Dateindex: yyyy/mos/day 
-Enter the most severe sanction that was given for the index offence/index cluster. If the individual was imprisoned for their offence this is always the most severe sanction. The sanction will be listed under Disposition on the CPIC in the third column.

\section{-Enter Sanction:}

0=Other sanction (e.g., NCR, fine)

1=Probation (this includes a suspended sentence- just enter the probation time)

2=House arrest/open custody/conditional sentence

3=Incarceration (include time served here)

- Enter sanctiontime: For the most severe sanction enter the number of months the individual was sentenced to (e.g., 5 years of incarceration is 60 months).

General Scoring Rules for the Static 99

-Please ensure that you have the coding manual in front of you as well as the CPIC study

manual. This CPIC manual is only a guide to each of the items and more information is in the coding manual if any points of confusion emerge. Please consult the Static-99R manual if you need more information or something has come up in coding the Static-99R that is confusing. If you're still unclear after reading the manual score it the best you can and mark the specific issue in the excel worksheet and consult the FAQ section. Also include how you addressed it in the scoring of the database. The PI will make sure to follow-up on this record. Points of disagreement will be dealt with by a consensus between the PI and RA. If a consensus cannot be reached the PI will consult with her supervisors on the best way to score the item.

-You must have a copy of the CPIC and Michael's report prior to scoring the Static-99R.

-Remember if you are unable to find the index offence (the offence for which they were referred to CAMH for) on the CPIC then flag the name in the Excel worksheet. Put all 
file material away and start back at step 1. For self-report or undetected offenders remember to skip to step 3 .

-If the report from Michael Kuban is missing check CHARTMAXX for an electronic copy of the report/psychological assessment. In the case of a missing report we can use a report on this system as we're just trying to find a report that will verify what the index offence is (why they were referred to CAMH). If you cannot find this information flag this in the Excel worksheet. Put file material away and start back at step 1.

-If you have exhausted all of the above you can put away the file information and return the CPIC. Please flag this person in the excel worksheet and start back at step 1

-In summary, in order to proceed to data entry you must have done the following: 1) Located all file information 2) Ensured that all information is consistent with the name and DOB on the CPIC 3) Have determined the index offence. If you are not able to meet 1-3 flag this in the Excel worksheet, return the file information to its correct place, and return to step 1. -You are to ignore anything after the index offence. Do not pay any attention to this when scoring the Static-99R (it may actually help to cover this info on the CPIC). Remember we wouldn't have had this information at the original assessment and the Static-99R is scored based on the index and any prior information we had on the offender.

Item 1: Age at release from index offence

-Material: CPIC

-Look at the individual's CPIC at the index offence and figure out the age of release by using their DOB.

-The date of release is the age when they have the first opportunity to reoffend. You need to know where the person is in their sentence when they are assessed at CAMH to calculate: 
- If the individual being assessed is still in jail calculate how old they will be when they're released back to the community and use this as age at release.

-If they are already in the community (e.g., under a supervision order) take their age at the date of assessment at CAMH as they currently have an opportunity to reoffend.

\section{-Enter S99agerel:}

\section{$18-34.99=1$}

\section{$35-39.99=0$}

\section{$40-59.99=-1$}

$60+=-3$

Item 2: Ever lived with an intimate partner

-Material: Michael's report/ChartMAXX

-This will be the trickiest item to score on the Static-99R as there will be situations where we do not have access to this information. If there is other report information included in the file with Michael's report (e.g., psychological assessment) skim this to see if you can find the information.

-Remember the question is asking if the individual has ever lived with an intimate partner for two years or more. If they have lived with someone for less than 2 years or were married for less than 2 years the answer is no $(0)$.

-If the individual is celibate or a priest and has not had a live in relationship the item is scored as the individual has never lived with a partner (1).

-If the individual is young or has been incarcerated for the majority of their life and they have not had a live in relationship for 2 years or more the item is scored as the individual has never lived with a partner (1). 
-Victims do not count toward this item. If the individual is living with someone under the age of consent, regardless of the context the item is scored as no. Here is more information on if the individual lived with a victim over the age of consent:

-“Generally, relationships with adult victims do not count. However, if the offender and the victim had two years of intimate relationship before the sexual offences occurred then this relationship would count, and the offender would score a " 0 " on this item. However, if the sexual abuse started before the offender and the victim had been living together in an intimate relationship for two years then the relationship would not count regardless of its length.” (pg. 25)

\section{-Enter S99intpar:}

$1=$ never had a live in intimate relationship for $\mathbf{2}$ or more years

$0=$ had live in intimate relationship for 2 or more years

$-1=$ Married at some point but unsure how long the marriage lasted

$-2=$ Never been married and there is not enough information to score the item (e.g., says

they are in a relationship but not sure how long)

Item 3: Index non-sexual violence- any convictions

-Material: CPIC/Michael's report

-This item is only referring to the index offence or index cluster and is only referring to non$\underline{\text { sexual violent offences. }}$

-In general on the STATIC-99R violent offences are only scored if there is a conviction. If there is anything less than a conviction attached to non-sexual violence we score this item as a no (0). 
-In order to count an offence towards the index non-sexual violence item it had to be dealt with on the same disposition date as the index sexual offence.

-This means that on the CPIC you will need to look in the Charge-Accusation $2^{\text {nd }}$ column and the third column to ensure there is a disposition attached to it.

-If it is only in the $4^{\text {th }}$ column (date, place, charge, and disposition) it does not count as these signify charges only.

-See page 27/28 of the CPIC manual for examples of non-sexual violence. A general rule is that anytime the word "gross" or "indecency" is included alongside an entry on the CPIC this means it is a sexual offence and is not scored as non-sexual violence. Instead it would be scored as an index sexual offence.

-Do not count driving offences and/or offences that were successfully appealed. Do not count a weapons offence unless it is clear from the title of the offence that the weapon was used against the victim (e.g., possession of a firearm does not count, but pointing a firearm does count). Resisting arrest does not count towards this item. All of these offences would be scored (0). -There will be some situations where the offence will be counted as both sexual and non-sexual violence:

-If it becomes apparent from Michael's report that the non-sexual index violence was sexually motivated (e.g., forcible confinement in the context of a sexual offence), this is coded under non-sexual violence \& sexual violence. It is very important to be $100 \%$ certain here that the non-sexual violent offence was sexually motivated. You will only get this information from CHARTMAXX and/or Michael's report. If you are unsure don't count it towards index sexual offending.

\section{-Enter S99indviol:}




\section{1= Index non-sexual violence}

\section{$0=$ No index non-sexual violence}

Item 4: Prior non-sexual violence- any convictions

-Material: CPIC/Michael's report

-This item is referring to anything prior to the index offence ( that was part of the index).

-In general on the STATIC-99R violent offences are only scored if there is a conviction. In cases where there is a charge or it is dropped this does not count and the item should be scored as a no (0).

-This means that on the CPIC you will need to look in the Charge-Accusation column and the Disposition third column to ensure there is a disposition attached to it. -If it is only in the $4^{\text {th }}$ column (date, place, charge, and disposition) it does not count as these signify charges only.

-See item 3 for exclusions and rules as significant overlap with item 3 in terms of what counts and what doesn't count for this item

-There will be some situations where the offence will count as both sexual and non-sexual violence:

-See item 3 for instructions on this but remember here this only counts for prior nonsexual violence. If they have forcible confinement that occurred in the context of a past sexual offence the individual is given one risk point for prior non-sexual violence and this also contributes to the score for prior sexual offence.

\section{-Enter S99priornvio:}

\section{$1=$ Prior to index non-sexual violence}




\section{$0=$ No prior to index non-sexual violence}

Item 5: Prior sexual offences- any charges or convictions

-Material: CPIC

-Remember to sum the charges and convictions separately for this item. Only count those sexual offences that occur prior to the index offences. If there are no prior sexual offences the score for this item is (0).

-Convictions look in the $2^{\text {nd }}$ column as these all have dispositions attached to them.

-Charges look in the fourth column

-Acquittals, not guilty findings, successful appeals still count as charges and are scored under this item.

-If a sex offence is appealed successfully this will show on the CPIC and should count only towards charges (not convictions)

-Prison misconduct for sexual acting out count as one charge per sentence regardless of how many charges there are for one disposition. This should only be coded if the sexual activity was coerced.

-Giving alcohol to a minor or providing noxious substances can only be counted if we know that it is in the context of facilitating a sexual offence, otherwise it is not counted on the Static-99R. To be sure we have to have information that it took place alongside the sexual offence either from the other charges that accompany it and/or Michael's report. You need to be $100 \%$ certain to score this as prior sexual offence.

-Count each sexual assault charge as one charge/conviction even if they occur on the same sentencing date. For example, if there are 10 charges of sexual assault and 8 convictions these counts at 10 charges and 8 convictions even if they are the same victim. 
-If the offender violates probation or parole with sexually offensive misbehaviour (a behaviour that generally would get a non-sexual offender in trouble with the law) this counts as a charge: -If the offender commits a sexual parole violation in July and another one in May count these as 2 charges. This differs from if the offender is charged with multiple sexual parole/probation violations at the same time count this as one charge.

-Technical violations do not count here (e.g., parole revoked for being in the presence of children). This would be suspicious activity, but for our purposes we are only looking at those items that would get someone into trouble if they were a non-offender.

-To get a score for this item take whatever sum is higher (charges or convictions). For example, if there are 2 charges, but 3 convictions the final score would be 2 :

\begin{tabular}{|l|l|l|}
\hline Charges & Convictions & Enter S99priorsex \\
\hline None & None & $\mathbf{0}$ \\
\hline $1-2$ & 1 & $\mathbf{1}$ \\
\hline $3-5$ & $2-3$ & $\mathbf{2}$ \\
\hline $6+$ & 4 & $\mathbf{3}$ \\
\hline
\end{tabular}

Item 6: Prior Sentencing Date

-Material: CPIC

-For this item only count sentencing dates (where there is a sanction). Look at the $2^{\text {nd }}$ column of the CPIC to gather this information.

-Court appearances that resulted in a not guilty for all offences, stayed offences, a complete acquittal, or convictions overturned on appeal are not counted towards prior sentencing dates. -If there is a conditional release and/or technical violation (e.g., drinking alcohol) these do not count as sentencing dates. 
-Do not count institutional rule violations even if it could have counted as a new offence.

-Do not count driving offences unless there are serious sanctions attached such as DUI, or reckless driving resulting in death or injury.

-Failure to appear does not count as a sentencing date. It is only when the individual appears and there is a conviction. Failure to register as a Sexual Offender does not count as a sexual offence. -Suspended sentences count as a sentencing date.

\section{-Enter S99priorsent:}

\section{$1=4$ or more separate sentencing dates}

\section{$0=$ Less than 4 sentencing dates}

Item 7: Any convictions for non-contact sexual offences

-Material: CPIC/Michael's Report

-When scoring this item keep in mind the difference between a category A versus category B sexual offence (see the manual for more information). For this item you are only to look at the $2^{\text {nd }}$ and $3^{\text {rd }}$ column of the CPIC (charge-accusation and disposition) as we only want convictions. -Non-contact sexual offence: Requires a conviction for a non-contact sex offence such as: exhibitionism, possession of obscene material, obscene telephone calls, voyeurism, indecent exposure (exhibitionism), elicit sexual use of the internet, sexual harassment

-Convictions for prowling, criminal trespassing or trespassing by night are often convictions for voyeurism. Look at Michael's report to determine whether this is related to voyeurism.

-There is a relatively new charge specific to electronic voyeurism, i.e., surreptitious recording of someone without their consent. This would count here as well. 
-If there was a non-contact sexual offence in the index it is a risk point for both non-contact offence and index sex offence.

-Where the offender tried to complete a contact sexual offence (e.g., attempted rape) code this as a contact sexual offence.

-Do not count prostitution or pimping offences

-Internet sex crimes are counted as non-contact sexual offences. Luring a child is a contact sexual offence, as we consider the intent as sexual contact.

-If the contact offence is originally charged and plea bargained down to a non-contact sex offence this counts as a contact sex offence.

-If a an offender with an indeterminate sentence is returned to prison for a non-contact sex offence that would result in a person from the general population receiving a charge, count this as a conviction for a non-contact sexual offence (be sure it would result in charges).

\section{-Enter S99ncsex:}

\section{$0=$ no non-contact sexual offence convictions}

\section{1= 1 or more non-contact sex offence conviction(s)}

Item 8/9/10 - Victim Items General Information

-Material: Michael's report/CHARTMAXX

-Any credible information counts here including admitted offending and any information on the record. This means that admissions, charges, convictions can all be counted here (basically anything in Michael's report regardless of whether it is on the CPIC).You'll mostly use Michael's report for victim information. You can check CHARTMAXX if you need prior information. 
-These three items are only applied to Category A sex offences (e.g., contact sex offences). For this item we do not score Category B sex offences (CP, prostitution, public sex). This is important to keep in mind because even though we will score the Static-99 on Category B offenders for those offenders they will automatically get scores of $(0)$ for item 8,9 , and 10 . Here $\underline{\text { is more information from the manual: }}$

- "Category "A" involves most criminal charges that we generally consider "sexual offences" and that involve an identifiable child or non-consenting adult victim. This category includes all contact offences, exhibitionism, voyeurism, sex with animals and dead bodies. Category " $\mathrm{B}$ " offences include sexual behaviour that is illegal but the parties are consenting or no specific victim is involved. Category "B" offences include prostitution related offences, consenting sex in public places, and possession of pornography. Behaviours such as urinating in public or public nudity associated with mental impairment are also considered Category "B" offences. For us the majority of category b offenders will be those involved with child pornography. Rule: if the offender has any category "A" offences on their record - all category "B" offences should be counted as sex offences for the purpose of scoring sexual priors or identifying the Index offense. They do not count for the purpose of scoring victim type items." (page 14 of the manual)

-Accidental victims: Page 48 includes information on this, but in general we only want to score intended victims. For example, if someone is an exhibitionist and they expose to a group of children (male and female) but the intended victims are female we do not count the male victims towards item 10. When it is ambiguous who the intended target was, assume that it was a female. 
-You can score charges, acquittals, not guilty findings and so forth. Generally we would want to look at file information on these charges to be sure that they are credible; however, we likely won't have this information. For our purposes we'll just score this as a victim.

-Sexual offences against animals do not count.

-If there is a sexual offence against a human (body) this does count and you should consult the training manual for further details.

-Count stayed offence information as well. Remember it is any credible information.

Item 8: Any unrelated victims

-Material: Michael's report

-Page 52-53 of the Static-99R coding manual has a long list of related victims. People who are unrelated for the purposes of the coding are step-parents/children of live-in parents that are less than 2 years, nephew's wife, second cousins, wife's aunt and so forth. If you are unsure whether someone is unrelated consult the coding manual.

-If the victim is a stranger the person automatically gets a point for any unrelated victim.

-Enter S99unrvic:

$0=$ no unrelated victims

$1=1$ or more unrelated victims

Item 9: Any stranger victims

-Material: Michael's report

-If they only have related victims (e.g., incest offender) you code them as no stranger victims (0)

-If they have an unrelated victim look further at Michael's report and the green sheet. We are looking for stranger victims (no previous relationship 24 hours prior to the sexual offence) 
-The classification of stranger is stringent and you should consult the manual if you are unsure. Given the rarity of stranger offences and that many in the study will have child victims, the majority of the time it is likely the victim will not be a stranger.

-In cases of stalking: if the victim does not know the offender it counts as a stranger offence. -In internet cases remember the 24 hour rule: If there has been sustained contact the victim does not count as a stranger. Remember to be stringent when scoring this item.

-People can become strangers over time (e.g., went to high school with the victim but 20 years has passed and they no longer know each other). This would count for this item.

-Enter S99strvic:

$0=$ no stranger victims

$1=1$ or more stranger victims

Item 10: Any Male Victim

-Material: Michael's report

-If there are any male victims score (1). This is always clear from Michael's report.

-Enter S99malevic:

$\mathbf{0}=$ no male victims

$1=1$ or more male victim

\section{Step 3: Score the Cormier Lang (offence severity)}

-Material: CPIC/Michael's report

-The Cormier-Lang is only to be scored for index/past criminal history (those offences prior to and including the index). It gives us an idea of offence severity.

-There will be two Cormier Lang Scores one for violent offences and one for non-violent offences. 
-Charges and convictions count towards the Cormier-Lang.

-If someone has multiple counts of the same offence on a disposition date multiply the Cormier-Lang value by the number of counts (e.g., 3 sexual assaults and sexual assault has a value of 10 on the Cormier-Lang it is $10 \times 3=30$ ).

-To score the Cormier Lang look at the Table Violent offences and allot points to each offence on their record. All Category A sex offenders should have a score for this item; however, if only indecent exposure then they will not have one for violence as this counts towards non-violence.

-For times where the individual could be scored a number of different ways for an offence (e.g., sexual assault which could be scored a 6 or a 10) and we don't have further information about what happened in the offence give the individual the lower score.

-If the offence is not listed in the table check the separate Cormier-Lang document on the $\mathrm{T}$ drive to see if someone has given a score for the offence. If this does not appear on the list score the new offence the same as a similar offence already listed in terms of the severity. Make sure you add this to the list in the T drive folder.

-Enter CLViolent - continuous variable (e.g., past history assault with a weapon score of 3 and sexual assault 10 gives them a score of 13)

Table. Cormier-Lang Violent Offences

\begin{tabular}{|l|c|}
\hline $\begin{array}{l}\text { Homicide (murder, manslaughter, criminal negligence causing } \\
\text { death) }\end{array}$ & 28 \\
\hline Attempted murder, causing bodily harm with intent to wound & 7 \\
\hline Kidnapping, abduction, and forcible confinement & 6 \\
\hline Aggravated assault, choking, administering a noxious thing & 6 \\
\hline Assault causing bodily harm & 5 \\
\hline
\end{tabular}




\begin{tabular}{|l|c|}
\hline Assault with a weapon & 3 \\
\hline Assault, assaulting a peace officer & 2 \\
\hline Aggravated sexual assault, sexual assault causing bodily harm & 15 \\
\hline Sexual assault with a weapon & 10 \\
\hline Sexual assault, gross indecency (vaginal or anal penetration; \\
victim forced to fellate offender)
\end{tabular}

-Now we will get a Cormier Lang Score for their non-violent offences. Remember this is index and anything prior to the index. Not all offenders will necessarily have a score on this item if they didn't commit non-violent offences.

-Enter CLNViol - (continuous variable)

Table. Non-violent Offences

\begin{tabular}{|l|c|}
\hline Robbery (bank, store) & 7 \\
\hline Robbery (purse snatching) & 3 \\
\hline Arson and fire setting (church, house, barn) & 5 \\
\hline Arson and fire setting (garbage can) & 1 \\
\hline Threatening with a weapon & 3 \\
\hline
\end{tabular}




\begin{tabular}{|l|c|}
\hline Threatening (uttering threats) & 2 \\
\hline Theft over (includes car theft and possession of stolen property over) & 5 \\
\hline Mischief to public or private property over & 5 \\
\hline Break and enter and commit an indictable offence (burglary) & 2 \\
\hline Theft under (includes possession of stolen property under) & 1 \\
\hline Mischief to public or private property under (includes public mischief) & 1 \\
\hline Break and enter (includes breaking and entering with intent to commit an & 1 \\
\hline offence & \\
\hline Fraud (extortion, embezzlement) & 5 \\
\hline Fraud (forged check, impersonation) & 1 \\
\hline Possession of a prohibited or restricted weapon & 1 \\
\hline Procuring a person for, or living on the avails of prostitution & 1 \\
\hline Trafficking in narcotics & 1 \\
\hline Dangerous driving, impaired driving (driving while intoxicated) & 1 \\
\hline Obstructing peace officer (including resist arrest) & 1 \\
\hline Causing a disturbance & 1 \\
\hline Wearing a disguise with the intent to commit an offence & \\
\hline Indecent exposure & 1 \\
\hline
\end{tabular}

\section{Step 4: Coding Index Victim Information}

-Material: Michal's report/ChartMAXX

-This information only pertains to the index offence. If it is an index cluster you will enter this information for the first three victims in the index offence. 
-For our purposes we will only be concerned about victim information pertaining to the index if the individual is a category A offender (in the index offence there is a contact sexual offence). If the individual is a category B offender do not enter victim information. Page 14 and 15 of the manual include a list of the different types of offences that fall under category A and B offences.

\section{-Enter CatOff:}

0= Category A offender

\section{1= Category $B$ offender}

-This information should be contained in Michael's report. If it is not contained in Michael's report check ChartMAXX to see if you can get police material and/or the full clinician report. If you exhaust these options code this as missing data (999) where there should be victim information, note it in the excel worksheet and proceed to step 4 . That is there will be missing information in this section potentially and it is okay to continue to enter data for them if we cannot enter all of this information. It is important to only record this victim information for victims where charges and convictions were the result (e.g., admissions, other unsuccessful investigations do not count here)

\section{-Enter victimnumb: enter the number of victims in the index offence (e.g., 5 victims).}

-Victim age: Here we want to know how old the victim was when the offence started and the offence ended. The reason there are two columns for this is that in many cases the abuse will have persisted over a number of years. If the abuse happened on one occasion enter the same age for when abuse started and when it ended.

-In cases of multiple victims for the index offence (i.e., offence cluster) remember to enter age at start of abuse and age at end of the abuse for each victim. 
-Enter Ind1 AgeS (continuous variable) first victim age at start of abuse

-Enter Ind1AgeF (continuous variable) first victim age at end of abuse

-Enter Ind2AgeS (continuous variable) second victim age at start of abuse if applicable

-Enter Ind2AgeF (continuous variable) second victim age at end of abuse if applicable

-Enter Ind3AgeS (continuous variable) third victim age at start of abuse if applicable

-Enter Ind3AgeF (continuous variable) third victim age at end of abuse if applicable

-Victim gender: Male or female victim in index offence. Again if it is an index cluster remember

to enter this information for each victim.

-Enter Ind1Gender

$0=$ male

$1=$ female

-Enter Ind2Gender and Enter Ind3Gender for victim 2 and 3 if applicable

-Victim-offender relationship: We will code this the same way as the Static-99R to keep things

consistent. The only difference is that stranger victim will be its own category. Again if it is an index cluster remember to enter this information for each victim.

-Enter Ind1rela

0=related

1=unrelated (includes stranger here)

2=stranger

-Enter Ind2rela and Ind3rela for victim 2 and 3 if applicable

Step 5: Coding Recidivism

-Material: CPIC only 
-Here we are using a hierarchical model to code recidivism. It is separated into: contact-sexual offences (any offence against a person that clearly involves unwanted or illegal sexual contact), non-contact sexual (any offence against a person where there is not direct sexual contact but there is sexual intent e.g., internet crimes, voyeurism), violent offences (any non-sexual offence against a person) and non-violent (includes all offences on the record that were not coded as violent or sexual). At the end of coding everything past the index offence on the CPIC should be included in the dataset. This includes acquittals, charges, stayed offences, and so forth.

$\underline{\text { Remember these only include offences that occur AFTER the index offence/index }}$ cluster. Do not count the index or anything prior to the index.

-We will also be recording the disposition date for first charge/conviction for each category

-We will be coding convictions and charges separately. Convictions are anything on the record that resulted in a criminal sanction $\left(2^{\text {nd }}\right.$ column $/ 3^{\text {rd }}$ column). Many times there will be multiple offences on a disposition date. The nature of each of these offences needs to be captured in the data. For example, if on one disposition date there is a conviction for 2 sexual contact offences and 1 property offence you would count 2 under contact sexual and 1 under non-violent. -Charges, withdrawn offences, stayed offences, appealed offence and so forth will be in the $4^{\text {th }}$ column under date, place charge and disposition. These need to be entered under charges. It will say what happened to the charge here (e.g., withdrawn). These are counted as charges.

Remember charges and convictions are separated.

-Make sure for appealed offences you don't double count (e.g., including the earlier conviction as well) 
-In cases where an unfamiliar charge arises and you are unsure where to code it please make a note in the Excel worksheet. Code it where you think it is most appropriate and just add this in the note. The PI will look into it and a decision will be made on a consensus.

-If you are unsure of a charge/conviction you can look it up in the criminal code in the T drive

-Please double check your counts after you've entered in the data for each column. This is likely where the majority of the mistakes will take place.

-We also want to note if we think there is pseudo recidivism on the record (e.g., historical charges that actually occurred PRIOR to the index but the individual is convicted after the index for these offences). The biggest clue here will be if the person has a criminal conviction after an index while they were still incarcerated (e.g., 27 year sentence and then the individual becomes a DO three years into his sentence). Please make sure you enter this in the Excel Datasheet as well and the data where you think there is pseudo recidivism

\section{-Enter pseudrec}

0=no

$1=$ yes

-Note that if the individual has died this is considered the end of their follow-up period as they don't have a chance to reoffend after that date. That means if they have not reoffended at all for each type you enter the date of death. If they have reoffended only for certain reoffence types enter the information as instructed below.

Sexual Contact Recidivism 
$\underline{\text {-Remember there will be a separate count for charges and convictions }}$

-Convictions include anything that appears on the record where there is a criminal sanction ( $2^{\text {nd }}$ column). If there is no sanction this will go under charges (e.g., not guilty, acquittals, charges dropped).

-We will use similar rule here for coding the STATIC-99R: If from the name of the offence it is implied that there is the intent of sexual contact code it under sexual contact recidivism. Only code this though if you are $100 \%$ sure.

-Remember there is a list of the majority of the Criminal Code sexual offences on the T drive. You can always tell the offence type by looking at the Criminal Code citation on the CPIC. When in doubt look up the information.

-Contact sexual offences in the Criminal Code are as follows:

-Sexual interference

-Invitation to sexual touching

-Sexual Exploitation of any kind (e.g., child, person with a disability)

-Sexual assault (e.g., sexual assault with a weapon, aggravated sexual assault, etc)

-Bestiality, Compelling the commission of bestiality, bestiality in presence of a child -Parent or guardian procuring sexual activity

-Incest

-Inference of sexual exploitation

-Luring a child

-Anything with the word attempted [contact sexual offences] score this as a contact sexual offence

-For including the recidivism disposition date you will include the month day and the year. 
-Enter in 2013/09/27 (date records retrieved) if they did not reoffend under the dates for both charges and conviction.

-If there are only charges that did not result in convictions the follow-up date for charges will be the disposition date and the follow-up date for convictions will be the end of the follow-up date.

-Enter sexcconv: total number of convictions (e.g., 3, 4, 5)

-Enter sexconvdate: Date of first sexual conviction after index yyyy/mos/day

-Enter sexccharge total number of charges (e.g., 3, 4, 5)

-Enter sexchardate: Date of first sexual charge after index yyyy/mos/day

Sexual Non-Contact Recidivism

$\underline{\text {-Remember there will be a separate count for charges and convictions }}$

-Convictions include anything that appears on the record where there is a criminal sanction. If there is no sanction this will go under charges (e.g., not guilty, acquittals, charges dropped).

-Offences

-Indecent acts/Exposure

-Voyeurism

-Corrupting morals

-Any child pornography offence

-Mailing obscene material

--For including the recidivism disposition date you will include the month day and the year. -Enter in 2013/09/27 (date records retrieved) if they did not reoffend under the dates for both charges and conviction. 
-If there are only charges that did not result in convictions the follow-up date for charges will be the disposition date and the follow-up date for convictions will be the end of the follow-up date.

-Enter sexncconv: total number of convictions (e.g., 3, 4, 5)

-Enter sexncconvdate: Date of first non-contact sexual conviction after index yyyy/mos/day

-Enter sexnccharge total number non-contact sex charges $(e . g ., 3,4,5)$

-Enter sexncchargedate: Date of first sexual charge after index yyyy/mos/day

Violent Recidivism

-Remember there will be a separate count for charges and convictions

-Convictions include anything that appears on the record where there is a criminal sanction. If there is no sanction this will go under charges (e.g., not guilty, acquittals, charges dropped).

-Violent offences involve action that involves harm against an identifiable human victim

-Offences

-Murder, manslaughter (any type including vehicular), infanticide

-Robbery

-Assault

-Criminal negligence

-Hostage taking

-Kidnapping, forcible confinement, abduction

-Only weapons offences in which it is clear there is intent to use it against the individual

(e.g., pointing a weapon)

-Any attempted violent offence 
-For including the recidivism disposition date you will include the month day and the year.

-Enter in 2013/09/27 (date records retrieved) if they did not reoffend under the dates for both charges and conviction.

-If there are only charges that did not result in convictions the follow-up date for charges will be the disposition date and the follow-up date for convictions will be the end of the follow-up date.

-Enter violconv: total number of convictions (e.g., 3, 4, 5)

-Enter violconvdate: First date of violent offence conviction after index yyyy/mos/day

-Enter violcharge: total number of charges (e.g., 3, 4, 5)

-Enter violchargeday: Date of first violent offence charge after index yyyy/mos/day

Non-Violent Recidivism

$\underline{\text {-Remember there will be a separate count for charges and convictions }}$

-Convictions include anything that appears on the record where there is a criminal sanction. If there is no sanction this will go under charges (e.g., not guilty, acquittals, charges dropped).

-Offences here are essentially left-overs from the above (non-violent recidivism; anything that doesn't involve sexual/violence). Some more confusing ones are provided as examples include: -Reckless driving or a driving conviction charged under the criminal code that does not result in injury to the other party

-Libel/hate propaganda

-Criminal harassment

-Arson

-Uttering threats 
-Any property offences

-Technical violations (e.g., failure to appear)

-Supplying noxious substances/supplying alcohol to a minor ->counted as general only if they do not occur in the context of another sexual offence charge

-Cruelty to animals

-Drug offences

-Resisting arrest

-Weapons offences that do not involve the individual using the weapon on another person (e.g., improperly storing a weapon)

-Prostitution/Bawdy house offences

-For including the recidivism disposition date you will include the month day and the year.

-Enter in 2013/09/27 (date records retrieved) if they did not reoffend under the dates for both charges and conviction.

-If there are only charges that did not result in convictions the follow-up date for charges will be the disposition date and the follow-up date for convictions will be the end of the follow-up date.

-Enter nvconv: total number of convictions (e.g., 3, 4, 5)

-Enter nviolconvdate: First date of non-violent conviction yyyy/mos/day

-Enter nvchargetotal number of charges (e.g., 3, 4, 5)

-Enter nvchargedate: First date of non-violent charge yyyy/mos/day

-Enter nvchargemos: month of first non-violent charge

Time spent in secure custody until first reoffence type 
-Enter Recdispconv: Number of disposition dates with conviction after the index (continuous variable).

-Remember if the individual has not reoffended the number of disposition dates is 0 -You only need to look at the convictions and disposition column ( $2^{\text {nd }}$ and $3^{\text {rd }}$ row) for this information as charges cannot result in prison time

\section{-Enter Recdispcha: Number of disposition dates with charges after the index (continuous variable).}

-Remember if the individual has not reoffended the number of disposition dates is 0

-You only need to look at the $4^{\text {th }}$ column for this info (remember to count each entry regardless of whether it resulted in a conviction)

-Again imagine you are the clinician on the assessment date that the offender comes in to see you. You are coding time spent in secure custody from the date of assessment until the end of the follow-up period (regardless of the nature of the charges).

-For individuals who have not served any prison time from CAMH assessment day until the end of their follow-up period: code 0's for all variables below.

-For individuals who have served prison time but have not reoffended at all code the total amount of prison time for each offence type (it will be consistent across all types of recidivism).

-For individuals who come to CAMH but are currently incarcerated you will need to figure out how much time remaining in their sentence they have left to serve. For example, an offender is convicted September $5^{\text {th }} 2005$ and comes to CAMH on December $5^{\text {th }} 2005$. The offender is serving a 12 month sentence with a release date of September $5^{\text {th }}$ 2006. This means that the offender has 9 months remaining in secure custody (12 
month sentence- 3 months already served prior to the assessment at CAMH). For this example you would need to count these 9 months towards the total time spent in secure custody until each of the first reoffence time calculations.

-For individuals who have served prison time you will need to calculate the total time spent in secure custody for each type of recidivism (this will likely vary by offence type). -If serving an indefinite sentence and/or NCR with no discharge noted on the record make sure you count all of the months from the date of the CAMH assessment until 2013/09/27 or until the first reoffence (can commit crimes in institutions).

-Enter mossex: number of months spent in secure custody (i.e., prison/jail; does not include probation or parole) until first sexual reoffence conviction

-Enter mossexcha: number of months spent in secure custody (i.e., prison/jail; does not include probation or parole) until first sexual reoffence charge (regardless of contact or non-contact)

-You will now enter the number of months spent in secure custody until first violent and first non-violent recidivism:

-Enter mosviol: number of months spent in secure custody (i.e., prison/jail; does not include probation or parole) until first violent reoffence conviction -Enter mosviolcha: number of months spent in secure custody (i.e., prison/jail; does not include probation or parole) until first violent reoffence charge -Enter mosnv: number of months spent in secure custody (i.e., prison/jail; does not include probation or parole) until first non-violent reoffence conviction -Enter mosnvcha: number of months spent in secure custody (i.e., prison/jail; does not include probation or parole) until first non-violent reoffence charge 


\section{Cormier-Lang for Recidivism}

-We will be scoring the Cormier-Lang violent and non-violent items for recidivism information as well to get an idea of how offence severity changes. This means you need to look at everything after the index to calculate these two scores. Please see item 3 for these charts and more important.

-Charges and convictions count towards the Cormier-Lang. It may be easiest to look at the charges (4th column) to get the score.

-If someone has multiple counts of the same offence on a disposition date multiple the Cormier-Lang value by the number of counts (e.g., 3 sexual assaults and sexual assault has a value of 10 on the Cormier-Lang it is $10 \times 3=30$ ).

-For times where the individual could be scored a number of different ways for an offence (e.g., sexual assault which could be scored a 6 or a 10) and we don't have further information about what happened in the offence give the individual the lower score.

-This will come up more when scoring recidivism because you won't have any idea what the nature of the offences are except from the CPIC label

-Consult the Cormier-Lang sheet on the $\mathrm{T}$ drive if an offence is not listed

-Enter Clviorec: Enter Cormier-Lang score for violent recidivism

-Enter Clnvrec: Enter Cormier-Lang score for non-violent recidivism (continuous variable)

\section{Step 6: IQ Scores}

-Material: Cognitive Report usually in the folder with Michael's report.

-This will only be in some of the offender's files. We are looking for a separate report included in the file. 
-If it is not there just leave this part of the dataset blank for that offender.

-The $3^{\text {rd }}$ or $4^{\text {th }}$ paragraph should include the type of IQ test administered and the full scale IQ score.

Enter IQ Score:

Enter Waistypev:

0=WAIS-R

1= WAIS III

2= WAIS IV

\section{Step 7: Finishing up}

-Check the information you have entered to ensure it is correctly entered.

-Enter in the excel sheet beside the name of the individual that the information has been entered.

If any problems/confusion emerged when entering the data please flag this in the excel

worksheet with a brief explanation of what the problem was. When in doubt always enter on the

Excel datasheet. This will highlight for the PI where common points of confusion emerge and/or tricky cases to go back to (e.g., cases of suspected pseudo recidivism).

-Save both the dataset and excel worksheet after entering data for an offender.

-Return the criminal record information to its correct place (alphabetical order). Make sure to return the file the correct place

-Close the individual's CHARTMAXX file if it was opened.

-Start back at step 1 for the next offender

Inter-Rater Reliability 
-Approximately $10 \%$ (88) cases will need to be entered a second time by another rater to ensure there is reliability between coders.

-When this takes place we will stop entering new cases until we've finished entering the cases we need to enter a second time

-Reliability checks will be carried out at three time points. 40 cases will be assessed early on followed by 24 in the middle and 24 at the end of data entry. These cases will be split between the RA and PI (e.g., of 40 cases the PI will check 20 and the RA will check 20). The first 40 cases will be scored for inter reliability. At case number 440, 24 cases will be checked and at the end of data collection 24 cases will be checked.

-For inter-rater reliability do not look at what the other person has entered. You are to go through all of the above steps as if you were scoring them for the first time.

-Note in the excel datasheet who you've entered the data for inter rater reliability. Again make note of any issues that came up when entering the data as per instructions in the coding manual. -Skye will run IRR check at three time points and meet with RAs if any major inconsistencies emerge. This is not to penalize anyone but rather to monitor rater drift throughout the study and ensure there is consistency. Please do not wait until this time though if there are points of confusion that come up. You should always make sure points of confusion are noted in the Excel worksheet. Skye will regularly check this worksheet and will get back to you with responses. You can also e-mail at any point about these concerns if there is no answer in the coding manual.

-FAQ document will be created for common sources of confusion as we progress with data entry. This will be stored in the file with the CPIC data. 


\section{References}

Ackerman, G., \& Beech, A. R. (2012). A systematic review of measures of deviant sexual interest and arousal. Psychiatry, Psychology and Law, 19, 118-143. doi: 10.1080/13218719.2010. 547161

Amelung, T., Kuhle, L.F., Konrad, A., Pauls, A., \& Beier, K. M. (2012). Androgen deprivation therapy of self-identifying, help-seeking pedophiles in the Dunkelfeld. International Journal of Law and Psychiatry, 35, 176-184. doi: 10.1016/j.iljp.2012.02.005

American Psychiatric Association. (2000). Diagnostic and statistical manual of mental disorders (4th ed., text rev.). Washington, DC: Author.

American Psychiatric Association. (2012). American Psychiatric Association DSM-5

Development. Retrieved from http://www.dsm5.org/Pages/Default.aspx.

American Psychiatric Association. (2013). Diagnostic and statistical manual of mental disorders (5th ed.). Washington, DC: Author.

Andrews, D. R., \& Bonta, J. (2010). Rehabiliting criminal justice policy and practice. Psychology Public Policy, and Law, 16, 39-55. doi: 10.1037/a0018362

Andrews, D. R., Bonta, J., \& Hoge, R. D. (1990). Classification for effective rehabilitation: Rediscovering psychology. Criminal Justice and Behavior, 17, 19-52. doi: $10.1177 / 0093854890017001004$

Archer, R. P., Buffington-Vollum, J. K., Vauter Sterdny, R., \& Handle, R. W. (2006). A survey of psychological test use patterns among forensic psychologists. Journal of Personality Assessment, 87, 84-94. doi: 10.1207/s15327752jpa8701_07

Babchishin, K. M., Nunes, K. L., \& Kessous, N. (2014). A multimodal examination of sexual interest in children: A comparison of sex offenders and nonsex offenders. Sexual Abuse: A Journal of Research and Treatment, 26, 343-374. doi: 10.1177/1079063213492343 
Banse, R., Schmidt, A., \& Clarbour, J. (2010). Indirect measures of sexual interest in child sex offenders: A multimethod approach. Criminal Justice and Behavior, 37, 319-355. doi: $10.1177 / 0093853809357598$.

Barbaree, H. E., \& Blanchard, R. (2008). Sexual deviance over the lifespan: Reduction in deviant sexual behaviour in the aging sex offender. In D.R. Laws \& W.T. O’Donohue (Eds.), Sexual deviance: Theory, assessment, and treatment (pp. 37-60). New York, NY: Guildford.

Barbaree, H. E., \& Marshall, W. L. (1989). Erectile response among heterosexual child molesters, father-daughter incest offenders, and matched non-offenders: Five distinct age preference profiles. Canadian Journal of Behavioural Science, 21, 70-82. doi:

$10.1037 / \mathrm{h} 0079791$

Batra, A. K., and Lue, T. F. (1990). Physiology and pathology of penile erection. Annual Review of Sex Research, 1, 251-263. doi: 10.1080/10532528.1990.10559863

Baxter, D. J., Marshall, W. L., Barbaree, H. E., Davidson, P. R., \& Malcom, P .B. (1984).

Deviant sexual behaviour: Differentiating sex offenders by criminal and personal history, psychometric measures, and sexual response. Criminal Justice and Behaviour, 11, 477501. doi: $10.1177 / 0093854884011004007$

Beier, K. M., Neutze, J., Mundt, I. A., Ahlers, C. J., Goecker, D., Konrad, A., . . Schaefer, G. A. (2009). Encouraging self-identified pedophiles and hebephiles to seek professional help: First results of the Prevention Project Dunkelfield (PPD). Child Abuse \& Neglect, 33, 543-549. doi: 10.1016/j.chiabu.2009.04.002

Beier, K. M., Amelung, T., Kuhle, L., Grundmann, D., Scerner, G., \& Neutze, J. (2013). Hebephilia as a sexual disorder. Fortschritte der Neurologie-Psychiatrie, 81, 128-137. doi: $10.1055 / \mathrm{s}-0032-1330539$ 
Blanchard, R. (2010). The DSM diagnostic criteria for pedophilia. Archives of Sexual Behavior, 39, 304-316. doi: 10.1007/s10508-009-9536-9536-0

Blanchard, R., Christensen, B. K., Strong, S. M., Cantor, J. M., Kuban, M. E., Klassen, P., . . . Blak, T. (2002). Retrospective self-reports of childhood accidents causing unconsciousness in phallometrically diagnosed pedophiles. Archives of Sexual Behavior, 31, 511-526. doi: 10.1023/A:1020659331965

Blanchard, R., Kolla, N. J., Cantor, J. M. Klassen, P. E., Dickey, R., Kuban. M. E., . . Blak, T. (2007). IQ, handedness, and pedophilia in adult male patients stratified by referral source. Sexual Abuse: A Journal of Research and Treatment, 19, 285-309. doi:10.1007/s1194007-9049-0

Blanchard, R., Klassen, P., Dickey, R., Kuban. M. E., \& Blak, T. (2001). Sensitivity and specificity of the phallometric test for pedophilia in nonadmitting sex offenders. Psychological Assessment, 13, 118-126. doi: 10.1037/1040-3590.13.1.118

Blanchard, R., Kuban, M. E., Blak, T., Klassen, P. E., Dickey, R., \& Cantor, J. M. (2012). Sexual attraction to others: A comparison of two models of alloerotic responding in men. Archives of Sexual Behavior, 41, 13-29. doi: 10.1007/s10508-010-9675-3

Blanchard, R., Kuban, M. E., Klassen, P., Dickey, R., Christensen, B. K., Cantor, J. M., ... Blak, T. (2003). Self-reported head injuries before and after age 13 for pedophilic and nonpedophilic men referred for clinical assessment. Archives of Sexual Behavior, 32, 573-581. doi: 10.1023/A:1026093612434

Blanchard, R., Kuban, M. E., Blak, T., Cantor, J. M., Klassen, P. E., \& Dickey, R. (2009). Absolute versus relative ascertainment of pedophilia in men. Sexual Abuse: A Journal of Research and Treatment, 21, 431-441. doi: 10.1177/1079063209347906 
Blanchard, R., Lykins, A. D., Wherrett, D., Kuban, M. E., Cantor, J. M., Blak, T., . . Klassen, P. E. (2009). Pedophilia, hebephilia, and the DSM-V. Archives of Sexual Behavior, 38, 335-350. doi: 10.1007/s10508-008-9399-9

Brouillette-Alarie, S., \& Proulx, J. (2014). An exploratory analysis of the offending process of extrafamilial sexual aggressors against adolescents. In Proulx, J., Beauregard, E., Lussier, P., Leclerc, B (Eds.), Pathways to sexual aggression (pp. 179-200). New York, NY: Routledge.

Brown, A. S., Gray, N. S., \& Snowden, R. J. (2009). Implicit measurement of sexual associations in child sex abusers: Role of victim type and denial. Sexual Abuse: A Journal of Research and Treatment, 21, 166-180. doi: 10.1177/107906320933234

Buss, D. M. (1994). The evolution of desire: Strategies of human mating. New York, NY: Basic Books.

Canales, D. D., Olver, M. E., \& Wong, S. C. P. (2009). Construct validity of the Violence Risk Scale-Sexual Offender Version for measuring sexual deviance. Sexual Abuse: A Journal of Research and Treatment, 21, 474-492. doi: 10.1177/107906320934490

Cantor, J. M. (2012). The errors of Karen Franklin's Pretextuality. International Journal of Forensic Mental Health, 11, 59-62. doi: 10.1080/14999013.2012.672945

Cantor, J. M., \& Blanchard, R. (2012). White matter volumes in pedophiles, hebephiles, and teleiophiles. Archives of Sexual Behaviour, 41, 749-752. doi: 19.1007/s10508-012-99542

Cantor, J. M., Blanchard, R., Christensen, B. K., Dickey, R., Klassen, P. E., Beckstead, A. L., . . . Kuban, M. E. (2004). Intelligence, memory, and handedness in pedophilia. Neuropsychology, 18, 3-14. doi: 10.1037/0894-4105.18.13 
Cantor, J. M., Kabami, N., Christensen, B. K., Zipursky, R. B., Barbaree, H. E., Dickey, R., , . Blanchard, R. (2008). Cerebral white matter deficiencies in pedophilic men. Journal of Psychiatric Research, 42, 167-183. doi: 10.1016/j.psychires.2007.10.013

Cantor, J. M., Klassen, P. E., Dickey, R., Christensen, B. K., Kuban, M. E., Blak, T., .. . N. S., \& Blanchard, R. (2005). Handedness in pedophilia and hebephilia. Archives of Sexual Behavior, 34, 447-459. doi: 10.1007/s10508-005-4344-7

Cantor, J. M., Kuban, M. E., Blak, T., Klassen, P. E., Dickey, R., Blanchard, R. (2006). Grade failure and special education placement in sexual offenders' educational histories. Archives of Sexual Behavior, 35, 743-751. doi: 10.1007/s10508-006-9018-6

Chivers, M. L., Seto, M. C., Lalumière, M. L, Laan, E., \& Grimbos, T. (2010). Agreement of genital and subjective measures of sexual arousal in men and women: A meta-analysis. Archives of Sexual Behavior, 39, 5-56. doi: 10.1007/s10508-009-9556-9

Cohen, J. (1988). Statistical power analysis for the behavioral sciences $\left(2^{\text {nd }}\right.$ ed.). Hillsdale, NJ Lawrence Erlbaum Associates

Duwe, G. (2013). Can Circles of Support and Accountability (COSA) work in the United States? Preliminary results from a randomized experiment in Minnesota. Sexual Abuse: A Journal of Research and Treatment, 25, 143-165. doi: 10.1177/1070-63212453942

Ebsworth, M., \& Lalumiére, M. L. (2012). Viewing time as a measure of bisexual sexual interest. Archives of Sexual Behaviour, 41, 161-172 doi: 10.1007/s10508-012-9923-9

Eke, A., \& Seto, M.C. (2011, March). Identifying recidivism risk factors for child pornography offenders. Paper presented at the $4^{\text {th }}$ International Congress on Psychology and Law, Miami, FL.

Elwood, R. W. (2009). Mental disorder, predisposition, prediction, and ability to control: 
Evaluating sexual offenders for civil commitment. Sexual Abuse: A Journal of Research and Treatment, 21, 395-411. doi: 10.1177/1079063209347723

Farrington, D.P. (1986). Age and crime. Crime and Justice, 7, 189-250.

Faul, F., Erdfelder, E., Lang, A. G., \& Buchner, A. (2007). G*Power 3: A flexible statistical power analysis program for the social, behavioral, and biomedical sciences. Behavior Research Methods, 39, 175-191. doi: 10.3758/BF03193146

Fedoroff, J. P., Kuban, M., \& Bradford, J. M. (2009). Laboratory measures of penile response in the assessments of sexual interest. In F.M Saleh, A.J. Grudzinskas, J.M. Bradford, \& D.J. Brodsky (Eds.), Sex offenders: Identification, risk assessment, treatment, and legal issues (pp. 89-100). New York, NY: Oxford University Press.

Field, A. (2013). Discovering statistics using IBM SPSS statistics. London, England: Sage.

Firestone, P., Bradford, J. M., McCoy, M., Greenberg, D. M., Curry, S., \& Larose, M. R. (2000). Prediction of recidivism in extrafamilial child molesters based on court-related assessments. Sexual Abuse: A Journal of Research and Treatment, 12, 203-221. doi: 1079-0632/00/0700-0203

Franklin, K. (2009). The public policy implications of "Hebephilia": A response to Blanchard et al. 2008. Archives of Sexual Behaviour, 38, 319-320. doi: 10.1007/s10508-008-9425-y

Franklin, K. (2010). Hebephilia: Quintessence of diagnostic pretextuality. Behavioral Sciences and the Law, 28, 751-768. doi: 10.1002/bsl.934

Freund, K. (1963). A laboratory method for diagnosing predominance of homo- and heteroerotic interest in the male. Behaviour Research and Therapy, 1, 85-93. doi: $10.1016 / 0005-7967(63) 90012-3$

Freund, K. (1965). Diagnosing heterosexual pedophilia by means of a test for sexual interest. 
Behaviour Research and Therapy, 3, 229-234. doi: 10.1016/0005-7967(65)90031-8

Freund, K. (1978). A conceptual framework for the study of anomalous erotic preferences. Journal of Sex and Marital Therapy, 4, 3-10. doi: 10.1080/00926237808402998

Freund, K. (1991). Reflections on the development of the phallometric method of assessing erotic preferences. Annals of Sex Research, 4, 221-228. doi: 10.1007/BF00850054

Freund, K., \& Blanchard, R. (1989). Phallometric diagnosis of pedophilia. Journal of Consulting and Clinical Psychology, 57, 100-105. doi: 10.1037/0022-006X.57.1.100

Freund, K., Chan, S., \& Coulthard, R. (1979). Phallometric diagnosis with "nonadmitters". Behaviour Research \& Therapy, 17, 451-457. doi: 10.1016/0005-7967(79)90061-5

Freund, K., Diamant, J., \& Pinkava, V. (1958). On the validity and reliability of the phalloplethysmographic diagnosis of some sexual deviations. Review of Czechoslovak Medicine, 4, 145-151.

Freund, K., Heasman, G., Rackansky, I. G., \& Glancy, G. (1984). Pedophilia and heterosexuality vs. homosexuality. Journal of Sex \& Marital Therapy, 10, 193-200. doi: $10.1080 / 00926238408405945$

Freund, K., \& Kuban, M. E. (1993). Deficient erotic gender differentiation in pedophilia: A follow-up. Archives of Sexual Behavior, 22, 619-628. doi: 10.1007/BF01543304

Freund, K., \& Langevin, R. (1976). Bisexuality in homosexual pedophilia. Archives of Sexual Behavior, 5, 415-423. doi: 10.1007/BF01541334

Freund, K., McKnight, C. K., Langevin, R., \& Cibiri, S. (1972). The female child as a surrogate object. Archives of Sexual Behavior, 2, 119-133. doi: 10.1007/BF01541862

Freund, K., \& Watson, R. (1991). Assessment of the sensitivity and specificity of a phallometric test: An update of "Phallometric diagnosis of pedophilia." Psychological Assessment, 3, 254-260. doi: 10.1037/1040-3590.3.2.254 
Freund, K., Watson, R., Rienzo, D. (1988). Signs of feigning in the phallometric test. Behavior Research and Therapy, 26, 105-112. doi: 10.1016/0005-7967(88)90109-X

Fritz, C. O., Morris, P. E., \& Richler, J. J. (2012). Effect size estimates: Current use, calculations, and interpretation. Journal of Experimental Psychology: General, 141, 2-18. doi: $10.1037 / \mathrm{a} 0024338$

Gebhard, P., Gagnon, J., Pomeroy, W., \& Christenson, C. (1965). Sex Offenders: An analysis of types. New York, NY: Harper \& Row.

Green, R. (2002). Is pedophilia a mental disorder? Archives of Sexual Behaviour, 31, 467-471. doi: 10.1023/A:1020699013309

Green, R. (2010). Sexual preference for 14-year-olds as a mental disorder: You can't be serious!! Archives of Sexual Behavior, 39, 585-586. doi: 10.1007/s 10508-010-9602-7

Glueck, B. C., Jr., (1955). Final report: Research project for the study and treatment of persons convicted of crimes involving sexual aberrations, June 1952 to June 1955. New York: New York State Department of Mental Hygiene.

Greenberg, D. M., Bradford, J. M., \& Curry, S. (1993). A comparison of sexual victimization in the childhoods of pedophiles and hebephiles. Journal of Forensic Science, 38, 432-437.

Guay, J., Proulx, J., Cusson, M., \& Ouimet, M. (2001). Victim-choice polymorphia among serious sex offenders. Archives of Sexual Behavior, 30, 521-533. doi: 10.1023/A:1010291201588

Hames, R., \& Blanchard, R. (2012). Anthropological data regarding the adaptiveness of hebephilia. Archives of Sexual Behaviour, 41, 745-747. doi: 10.1007/s10508-012-9972-0

Hanson, R. K., Bourgon, G., Helmus, L., \& Hodgson, S. (2009). The principles of effective 
correctional treatment also apply to sexual offenders: A meta-analysis. Criminal Justice and Behavior, 36, 865-891. doi:10.1177/0093854809338545.

Hanson, R. K., \& Bussiére, M. T. (1998). Predicting relapse: A meta-analysis of sexual offender recidivism studies. Journal of Consulting and Clinical Psychology, 66, 348-362. doi: 10.1037/0022-006X.66.2.348

Hanson, R. K., \& Morton-Bourgon, K. E. (2004). Predictors of sexual recidivism: An updated meta-analysis. (Research Rep. No. 2004-02). Ottawa, Canada: Public Safety and Emergency Preparedness Canada.

Hanson, R. K., Morton, K. E., \& Harris, A. J. R. (2003). Sexual offender recidivism risk: What we know and what we need to know. In R. A. Prentky, E. S. Janus, \& M. C. Seto (Eds.), Annals of the New York Academy of Sciences: Vol. 989. Sexually coercive behavior: Understanding and management (pp. 154-166). New York: New York Academy of Sciences.

Hanson, R. K., \& Morton-Bourgon, K. E. (2009). The accuracy of recidivism risk assessments for sexual offenders: A meta-analysis of 118 prediction studies. Psychological Assessment, 21, 1-21. doi: 10.1037/a0014421

Hanson, R. K., \& Morton-Bourgon, K. E. (2005). The characteristics of persistent sexual offenders: A meta-analysis of recidivism studies. Journal of Consulting and Clinical Psychology, 73, 1154-1163. doi: 10.1037/0022-006X.73.6.1154

Hanson, R.K., \& Thornton, D. (2000). Improving risk assessments for sex offenders: A comparison of three actuarial scales. Law and Human Behavior, 24, 119-136. doi: 10.1023/A:100548291333

Harris, G. T., \& Rice, M. E. (1996). The science in phallometric measurement of male sexual 
interest. Current Directions in Psychological Science, 5, 156-160.

Harris, G. T., \& Rice, M. E. (2007). Adjusting actuarial violence risk assessments based on aging or the passage of time. Criminal Justice and Behavior, 34, 397-313. doi:

$10.1177 / 0093854806293486$

Harris, G. T., Rice, M. E., Quinsey, V. L., Chaplin, T. C., \& Earls, C. (1992). Maximizing the discriminant validity of phallometric assessment data. Psychological Assessment, 4, 502511. doi: $10.1037 / 1040-3590.4 .4 .502$

Heil, P., Ahlmeyer, S., \& Simons, D. (2003). Crossover sexual offences. Sexual Abuse: A Journal of Research and Treatment, 15, 221-236. doi: 10.1177/107905320301500401

Helmus, L., Hanson, R. K., Thornton, D., Bachishin, K. M., \& Harris, A. J. R. (2012). Absolute recidivism rates predicted by Static-99R and Static-2002R sex offender risk assessment tools vary across samples: A meta-analysis. Criminal Justice and Behavior, 39, 11481171. doi: $10.1177 / 0093854812443648$

Helmus, L., Thornton, D., Hanson, R. K., Babchishin, K. M. (2012). Improving the predictive accuracy of Staitc-99 and Staitc-2002 with older sex offenders: Revised age weights. Sexual Abuse: A Journal of Research and Treatment, 24, 64-101. doi: $10.1177 / 1079063211409951$

Hemphill, J. F. (2003). Interpreting the magnitudes of correlation coefficients. American Psychologist, 58, 78-80. doi: 0003-066X.58.1.78

Herman-Giddens, M. E., Slora, E. J., Wasserman, R. C., Bourdony, C. J., Bhapkar, M. V., Koch, G. G., ... Hasemeir, C. M. (1997). Secondary sexual characteristics and menses in young girls seen in office practice: A study from the pediatric research in office settings network. Pediatrics, 99, 505-512. doi: 10.1542/peds.99.4.505 
Herman-Giddens, M. E., Wang, L., Koch, G. (2001). Secondary sexual characteristics in boys: Estimates from the National Health and Nutrition Examination Survey III, 1988-1994. Archives of Pediatric and Adolescent Medicine, 155, 1022-1028. doi:

10.1001/archpedi.155.9.1022

Hildebrand, M., de Ruiter, C., \& de Vogel, V. (2004). Psychopathy and sexual deviance in treated rapists: Association with sexual and nonsexual recidivism. Sexual Abuse: A Journal of Research and Treatment, 16, 1-23. doi: 10.1023/B:SEBU.0000006281. 93245.de

Howes, R.J. (1995). A survey of plethysmographic assessment in North America. Sexual Abuse: A Journal of Research and Treatment, 7, 9-24. doi: 10.1177/107906329500700104

Janssen, D. F. (2009). Hebephilia plethysmographica: A partial rejoinder to Blanchard et al. (2008). Archives of Sexual Behaviour, 38, 321-322. doi: 10.1007/s10508-009-9479-5

Jespersen, A. F., Lalumiére, M., Seto, M.C. (2009). Sexual abuse history among adult sex offenders and non-sex offenders: A meta-analysis. Child Abuse \& Neglect, 33, 179-192. doi: 10.1016/j.chiaabu.2008.07.004

Kenrick, D. T., \& Keefe, R. C. (1992). Age preferences in mates reflect sex differences in human reproductive strategies. Behavioral and Brain Sciences, 15, 75-133. doi: $10.1017 / \mathrm{S} 0140525 \mathrm{X} 00067595$

Kingston, D. A., Firestone, P., Moulden, H. M., \& Bradford, J. M. (2007). The utility of the diagnosis of pedophilia: A comparison of various classification procedures. Archives of Sexual Behaviour, 36, 423-436. doi: 10.1007/s10508-006-9091-x

Kingston, D. A., Firestone, P., Wexler, A., \& Bradford, J. M. (2008). Factors associated with 
recidivism among intrafamilial child molesters. Journal of Sexual Aggression, 14, 3-18. doi: $10.1080 / 13552600802074924$

Kramer, K.L. (2008). Early sexual maturity among Pume foragers of Venezuela: Fitness implications of teen motherhood. American Journal of Physical Anthropology, 136, 338350. doi: 10.1002/ajpa.20817

Kuban, M., Barbaree, H. E., \& Blanchard, R. (1999). A comparison of volume and circumference phallometry: Response magnitude and method agreement. Archives of Sexual Behavior, 28, 345-359. doi: 10.1023/A:1018700813140

Lalumiére, M. L., \& Harris, G. T. (1998). Common questions regarding the use of phallometric testing with sexual offenders. Sexual Abuse: A Journal of Research and Treatment, 10, 227-237. doi: 1079-0632/98/0700-0227

Laws, R. D. (1994). How dangerous are rapists to children? The Journal of Sexual Aggression, 1, 1-14. doi: $10.1080 / 13552609408413238$

Laws, R. D. (2009). Penile phelthysmography: Strengths, limitation, innovations. In D. Thornton, \& R. D. Laws (Eds.), Cognitive approaches to the assessment of sexual interest in sexual offenders (pp. 7-29). Malden, MA: John Wiley \& Sons.

Laws, R. D, Hanson, R. K., Osborn, C. A., \& Greenbaum, P. E. (2000). Classification of child molesters by plethsmographic assessment of sexual arousal and a self-report measures of sexual preference. Journal of Interpersonal Violence, 14, 1297-1312. doi: $10.1177 / 088626000015012004$

Levenson, J. S., Becker, J., \& Morin, J. W. (2008). The relationship between victim age and gender crossover among sex offenders. Sexual Abuse: A Journal of Research and Treatment, 20, 43-60. doi: 10.1177/107906320831418

Looman, J., Morphett, A. C. C., \& Abracen, J. (2012). Does consideration of psychopathy and 
sexual deviance add to the predictive validity of the Static-99R? International Journal of Offender Therapy and Comparative Criminology, 57, 939-965. doi:

\section{$10.1177 / 036624 X 12444839$}

Lussier, P., \& Davies, G. (2011). A person-oriented perspective on sexual offenders, offending trajectories, and risk of recidivism: A new challenge for policymakers, risk assessors, and actuarial prediction? Psychology, Public Policy, and Law, 17, 530-561. doi: $10.1037 / \mathrm{a} 0024388$

Lussier, P., Leclerc, B., Healey, J., \& Proulx, J. (2007). Generality of deviance and predation: Crime-switching and specialization patterns in persistent sexual offenders. In M. Delisi, \& P. J. Conis (Eds.), Violent offenders: Theory, public policy and practice (pp. 97-118). Boston, MA: Jones and Bartlett Publishers.

Lykins, A. D, Cantor, J. M., Kuban, M. E., Blak, T., Dickey, R., Klassen, P. E., . . Blanchard, R. (2010a). Sexual arousal to female children in gynephilic men. Sexual Abuse: A Journal of Research and Treatment, 22, 279-2891. doi: 10.1177/1079063210372141

Lykins, A.D, Cantor, J. M., Kuban, M. E., Blak, T., Dickey, R., Klassen, P. E., . . Blanchard, R. (2010b). The relation between peak response magnitudes and agreement in diagnoses obtained from two different phallometric tests for pedophilia. Sexual Abuse: A Journal of Research and Treatment, 22, 41-57. doi: 10.1177/1079063209352094

Mackaronis, J. E., Strassberg, D. S., \& Marcus, D. K. (2011). The latent structure of multiphasic sex inventory- Assessed pedophilic interest. Psychological Assessment, 23, 1017-1022. doi: $10.1037 / \mathrm{a} 002465$

Mann, R. E., Hanson, R. K., \& Thornton, D. (2010). Assessing risk for sexual recidivism: Some 
proposals on the nature of psychologically meaningful risk factors. Sexual Abuse: A Journal of Research and Treatment, 22, 191-217. doi: 10.1177/1079063210366039

McCann, K., \& Lussier, P. (2008). Antisociality, sexual deviance, and sexual reoffending in juvenile sex offenders: A meta-analytical investigation. Youth Violence and Juvenile Justice, 6, 363-385. doi: 10.1177/1541204008320260

McGrath, R., Cumming, G., Burchard, B., Zeoli, S., \& Ellerby, L. (2010). Current practices and emerging trends in sexual abuser management: The safer society 2009 North American survey. Brandon, VT: Safer Society Press

Merdian, H. L., \& Jones, D. T. (2011). Phallometric assessment of sexual arousal. In D. P. Boer, R. Eher, L. A. Craig, M. H. Miner, \& F. Pfafflin (Eds.), International perspectives on the assessment and treatment of sexual offenders (pp.141-169). West Sussex, UK: Wiley \& Sons.

Michaud, P., \& Proulx, J. P. (2009). Penile-response profiles of sexual aggressors during phallometric testing. Sexual Abuse: A Journal of Research and Treatment, 21, 308-336. doi: $10.1177 / 1079063209342073$

Moser, C. (2009). When is an unusual sexual interest a mental disorder? Archives of Sexual Behaviour, 38, 323-325. doi: 10.1007/s10508-008-9436-8.

Murphy, W. D., \& Barbaree, H. E. (1994). Assessments of sex offenders by measures of erectile response: Psychometric properties and decision making. Brandon, VT: The Safer Society Press.

Moulden, H. M., Firestone, P., Kingston, D., \& Bradford, J. (2009). Recidivism in pedophiles: An investigation using different diagnostic methods. The Journal of Forensic Psychiatry \& Psychology, 20, 68-701. doi: 10.1080/14789940903174055 
O'Donohue, W. T., \& Letourneau, E. (1992). The psychometric properties of the penile tumescence assessment of child molesters. Journal of Psychopathology and Behavioral Assessment, 14, 123-174. doi: 10.1007/BF00965172

Olver, M. E., \& Wong, S. C. P. (2006). Psychopathy, sexual deviance, and recidivism among sex offenders. Sexual Abuse: A Journal of Research and Treatment, 18, 65-82. doi: $10.1007 / \mathrm{s} 11194-006-9006-3$

Parent, G., Guay, J-P., \& Knight, R. A. (2011). An assessment of long-term risk of recidivism by adult sex offenders: One size doesn't fit all. Criminal Justice and Behavior, 38, 188-209. doi: $10.1177 / 0093854810388238$

Plaud, J. J. (2009). Are there "hebephiles" among use? A response to Blanchard et al. (2008). Archives of Sexual Behaviour, 38, 326-327. doi: 10.1007/s10508-008-9423-0

Porter, S., Fairweather, D., Drugge, J., Herve, H., Brit, A., Boer, D. (2000). Profiles of psychopathy in incarcerated sexual offenders. Criminal Justice and Behaviour, 27, 216233. doi: $10.1177 / 0093854800027002005$

Proulx, J., Côté, G., \&Achille, P. A. (1993). Prevention of voluntary control of penile response in homosexual pedophiles during phallometric testing. Journal of Sex Research, 30, 140147. doi: $10.1080 / 00224499309551694$

Proulx, J., Pellerin, B., Paradis, Y., McKibben, A., Aubut, J., \& Ouimet, M. (1997). Static and dynamic predictors of recidivism in sexual aggressors. Sexual Abuse: A Journal of Research and Treatment, 9, 7-27. doi: 10.1177/107906329700900102

Quinsey, V. L. \& Bergersen, S. G. (1976). Instructional control of penile circumference in assessments of sexual preference. Behavior Therapy, 7, 489-493. doi: 10.1016/S00057984(76)80168-2 
Quinsey, V. L., Rice, M. E., \& Harris, G. T. (1995). Actuarial prediction of sexual recidivism. Journal of Interpersonal Violence, 10, 85-105. doi: 10.1177/088626095010001006

Rice, M. E., \& Harris, G. T. (2005). Comparing effect sizes in follow-up studies: ROC Area, Cohen's d, and r. Law and Human Behavior, 29, 615-620. doi: 10.1007/s10979-005$6832-7$

Rice, M. E., Harris, G. T., Lang. C., \& Cormier, C. (2006). Violent sex offences: How are the best measured from official records? Law and Human Behavior, 30, 525-541. doi: 10.1007/s10979-006-9022-3

Rind, B., \& Yuill, R. (2012). Hebephilia as mental disorder? A historical, cross-cultural, sociological, cross-species, non-clinical empirical and evolutionary review. Archives of Sexual Behaviour, 41, 797-829. doi: 10.1007/s10508-012-9982-y

Robertiello, G., \& Terry, K. J. (2007). Can we profile sex offenders? A review of sex offender typologies. Aggression and Violent Behavior, 12, 508-518. doi:

10.1016/j.avb.2007.02.010

Ryniker, D. C. (2012). Hebephilia and male fertility. Archives of Sexual Behaviour, 41, 741-743. doi: $10.1007 / \mathrm{s} 10508-012-9977-8$.

Rusico, J., Haslm, N., Meron Ruscio, A. (2006). Introduction to the taxometric method: A practical guide. Mahwah, NJ: Lawrence Erlbaum Associates.

Schmidt, A. F., Mokros, A., \& Banse, R. (2013). Is pedophilic sexual preference continuous? A taxometric analysis based on direct and indirect measures. Psychological Assessment, 25, 1146-1153. doi: 10.1037/a0033326

Seto, M. C. (2001). The value of phallometry in the assessment of male sex offenders. Journal of Forensic Psychology Practice, 1, 65-75. doi: 10.1300/J158v01n02_05

Seto, M. C. (2002). Precisely defining pedophilia. Archives of Sexual Behavior, 31, 498-499. 
Seto, M. C. (2008). Pedophilia and sexual offending against children: Theory, assessment, and intervention. Washington, DC: American Psychological Association.

Seto, M. C. (2010). Child pornography use and Internet solicitation in the diagnosis of pedophilia [letter to the editor]. Archives of Sexual Behavior, 39, 591-593. doi:10.1007/s10508-0109603-6

Seto, M. C. (2013). Internet Sex Offenders. Washington DC: American Psychological Association.

Seto, M. C., Cantor, J. M., \& Blanchard, R. (2006). Child pornography offenses are a valid diagnostic indicator of pedophilia. Journal of Abnormal Psychology, 115, 610-615. doi: 10.1037/0021-843X.115.3.61

Seto, M. C., \& Eke, A. W. (2015). Predicting recidivism among adult male child pornography offenders: Development of the Child Pornography Offender Risk Tool (CPORT). Law and Human Behavior, Advance online publication. doi: 10.1037/lhb0000128

Seto, M. C., \& Lalumiére, M.L. (2001). A brief screening scale to identify pedophilic interests among child molesters. Sexual Abuse: A Journal of Research and Treatment, 13, 15-25. doi: 10/1177/107906320101300103

Seto, M. C., \& Lalumiére, M.L. (2010). What is so special about male adolescent sexual offending? A review and test of explanations through meta-analysis. Psychological Bulletin, 136, 526-574. doi: 10.1037/a0019700

Seto, M. C., Lalumiére, M. L., \& Kuban, M. E. (1999). The sexual preferences of incest offenders. Journal of Abnormal Psychology, 108, 267-272. doi: 10.1037/0021843X.108.2.267

Seto, M. C., Stephens, S., Lalumiére, M. L., \& Cantor, J. M. (2015). The revised Screening Scale 
for Pedophilic Interest- 2 (SSPI-2): Development and validation. Accepted pending minor revisions.

Singer, B. (1984). Conceptualizing sexual arousal and attraction. The Journal of Sex Research, 20, 230-240. doi: 10.1080/00224498409551222

Skorvan, L. C., Huss, M. T., \& Scalora, M. J. (2010). Sexual fantasies and sensation seeking among psychopathic sexual offenders. Psychology, Crime \& Law, 16, 617-629. doi: $10.1080 / 106831609022998025$

Studer, L. H., Aylwin, A. S., Clelland, S. R., Reddon, J. R.,\& Frenzel, R. R. (2002). Primary erotic age preference in a group of child molesters. International Journal of Law and Psychiatry, 25, 173-180. doi: 10.1016/S0160-2527(01)00111-X

Suchy, Y., Whittaker, W. J., Strassberg, D. S., Eastvold, A. (2009). Neurocognitive differences between pedophilic and non-pedophilic child molesters. Journal of the International Neuropsychological Society, 15, 248-257. doi: 1017/S1355617709090353

Swets, J. A. (1988). Measuring the accuracy of diagnostic systems. Science, 240, 1285-1293. doi: $10.1126 /$ science. 3287615

Tanner, J. M. (1978). Foetus into man: Physical growth from conception to maturity. Cambridge, MA: Harvard University Press.

Tromovitch, P. (2009). Manufacturing mental disorder by pathologizing erotic age orientation": A comment on Blanchard et al. (2008). Archives of Sexual Behaviour, 38, 328. doi: $10.1007 /$ s10508-008-9426-x

Wakefield, J. C. (1992). Disorder as harmful dysfunction: A conceptual critique of DSM-III-R's definition of mental disorder. Psychological Review, 99, 232-247.

Wakefield, J. C. (2012). The DSM-5's proposed new categories of sexual disorder: The problem 
of false positives in sexual diagnosis. Clinical Social Work Journal, 40, 213-223. doi: $10.1007 / \mathrm{s} 10615-011-0353-2$

Wilson, R. J., Abracen, J., Looman, J., Picheca, J. E., \& Ferguson, M. (2011). Pedophilia: An evaluation of diagnostic and risk prediction methods. Sexual Abuse: A Journal of Research and Treatment, 23, 260-274. doi: 10.1177/1079063210384277

World Health Organization. (1992). International statistical classification of diseases and related health problems (10th rev., Vol. 1). Geneva, Switzerland: Author. 